WSRC-TR-2003-00540

\title{
FY03 DNAPL Characterization of the A-14 Outfall
}

\author{
B. D. Riha \\ J. Rossabi \\ Savannah River Technology Center
}

September 2003

Westinghouse Savannah River Company, LLC Savannah River Site Aiken, SC, 29808

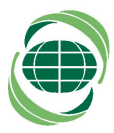


This document was prepared in conjunction with work accomplished under Contract No. DE-AC09-96SR18500 with the U. S. Department of Energy.

\section{DISCLAIMER}

This report was prepared as an account of work sponsored by an agency of the United States Government. Neither the United States Government nor any agency thereof, nor any of their employees, makes any warranty, express or implied, or assumes any legal liability or responsibility for the accuracy, completeness, or usefulness of any information, apparatus, product or process disclosed, or represents that its use would not infringe privately owned rights. Reference herein to any specific commercial product, process or service by trade name, trademark, manufacturer, or otherwise does not necessarily constitute or imply its endorsement, recommendation, or favoring by the United States Government or any agency thereof. The views and opinions of authors expressed herein do not necessarily state or reflect those of the United States Government or any agency thereof.

This report has been reproduced directly from the best available copy.

Available for sale to the public, in paper, from: U.S. Department of Commerce, National Technical Information Service, 5285 Port Royal Road, Springfield, VA 22161, phone: (800) 553-6847, fax: (703) 605-6900

email: orders@ntis.fedworld.gov

online ordering: http://www.ntis.gov/help/index.asp

Available electronically at http://www.osti.gov/bridge

Available for a processing fee to U.S. Department of Energy and its contractors, in paper, from: U.S. Department of Energy, Office of Scientific and Technical Information, P.O. Box 62, Oak Ridge, TN 37831-0062,

phone: (865)576-8401,

fax: (865)576-5728

email: $\underline{\text { reports@ adonis.osti.gov }}$ 


\section{Contents}

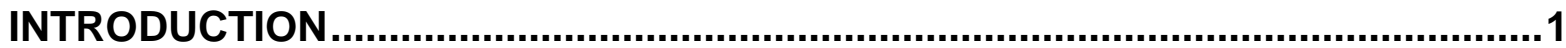

METHODS

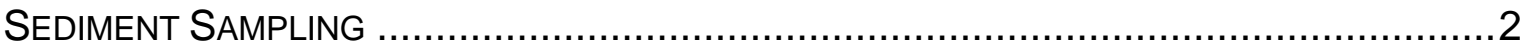

SEDIMENT HEADSPACE ANALYSIS FOR VOCS ...............................................

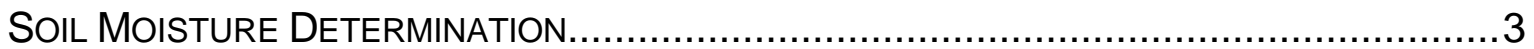

RESULTS AND CONTAMINANT DISTRIBUTION.............................................

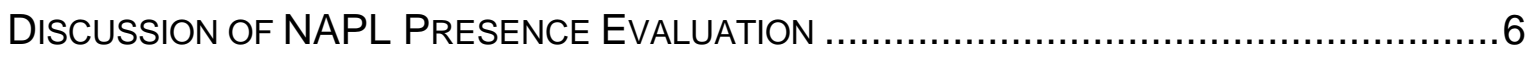

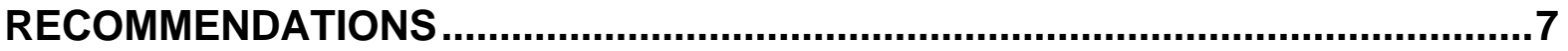

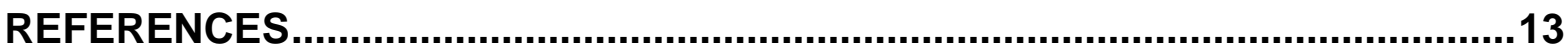

APPENDIX A - HEATED HEADSPACE GAS CHROMATOGRAPHY PHASE

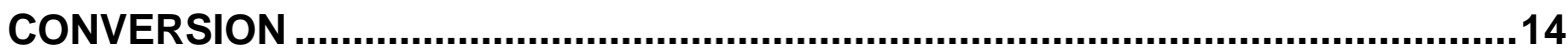

APPENDIX B - CR BORING SERIES ANALYTICAL RESULTS TABLES ............15 


\section{List of Figures}

Figure 1 - PCE Sediment Concentration Plume above $10 \mathrm{mg} / \mathrm{kg}$ at the A-14 Outfall.4

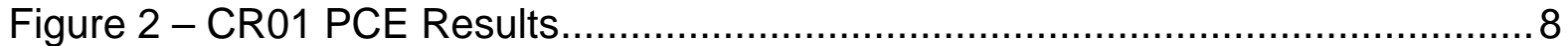

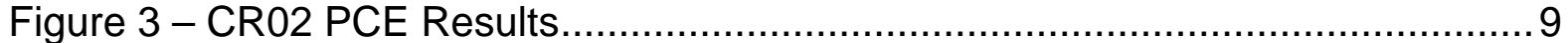

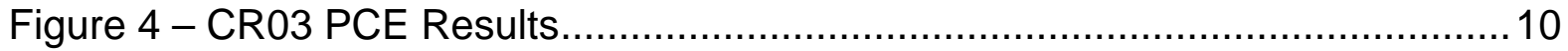

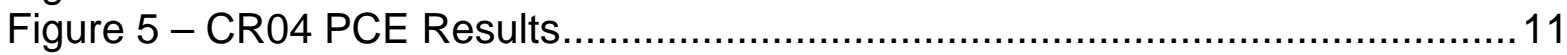

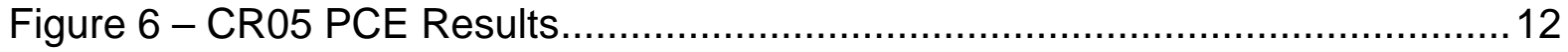




\section{List of Tables}

Table 1 - Sediment Analysis Detection Limits

Table 2 - SRS Coordinates for the Sediment Borings ......................................... 5

Table 3 - Soil Moisture Results for Boring CR1 ................................................ 5

Table 4 - Soil Moisture Results for Boring CR2 …............................................ 6

Table 5 - Theoretical Concentrations for the Presence of NAPL in the SRS Vadose Zone Sediments

Table 6 - Sediment Analysis Results for Boring CR01 ........................................15

Table 7 - Sediment Analysis Results for Boring CR02 …....................................20

Table 8 - Sediment Analysis Results for Boring CR03 …..................................27

Table 9 - Sediment Analysis Results for Boring CR04 .........................................31

Table 10 - Sediment Analysis Results for Boring CR05 ........................................3 


\section{Introduction}

Residual dense non-aqueous phase liquid (DNAPL) contamination continues to be one of the most challenging remediation and characterization problems at SRS and sites around the world. Chlorinated solvents were usually released as DNAPLs to the subsurface where they move in an unstable fashion driven by gravitational and capillary forces. They are often retained in small discrete blobs $\left(\ll<1 \mathrm{~m}^{3}\right)$ in fine grain materials in the vadose zone and contaminate ground water by slow continuous release through dissolution and diffusion. Locating these small sources is a difficult but crucial part of remediating a contaminated site. Several methods have been developed to locate subsurface DNAPL but nearly all are intrusive and can only identify DNAPL in close proximity to the access hole. Minimally invasive geophysical methods to locate residual DNAPL have been proposed and developed but few methods are capable of the spatial resolution required. Complex resistivity measurements sensitive to DNAPL (perchloroethylene) interactions with clay (smectite) have recently been shown to have promise in laboratory experiments. Based on these laboratory results, field tests of the complex resistivity technique were performed at the A-014 outfall.

To support the field testing of complex resistivity techniques, an area (approximately $10 \mathrm{~m} \times 15 \mathrm{~m}$ ) was selected with residual DNAPL confirmed by both the NAPL FLUTe (Ribbon NAPL Sampler) and cone penetrometer soil sampling and analysis. After complex resistivity measurements were made, 378 depth-discrete 1 foot long sediment cores at 5 selected locations within the test area were collected and analyzed to compare with the geophysical results. Despite inherent differences in spatial resolution and coverage and the limited number of baseline samples, this comparison can be used to provide some measure of performance of the geophysical technique.

Three separate groups of researchers (Blackhawk GeoServices/Colorado School of Mines; Lawrence Livermore National Laboratory; Massachusetts Institute of Technology) made complex resistivity measurements using electrodes installed in the test area. The DOE Office of Science and Technology (OST) funded the groups. SRTC was supported by both SGCP and OST to perform confirmation sampling to determine the performance of the geophysical technique.

Sediment samples were collected using a direct penetration rig (Gregg In Situ Rhino Rig) using one of two methods. Samples were collected at depth using either the Gregg In Situ Macro Core Sampler or the Vertek wireline sediment sampler provided to Gregg by SRTC. A pneumatic hammer system on the Rhino Rig was used to advance the penetration rods when resistance prevented penetration by static pushing. A total of 378 sediment cores from different depths in the vadose zone were collected and more than 700 sediment samples were analyzed (including duplicates) for VOCs. More than $15 \%$ of the 378 cores were determined to contain residual NAPL. 


\section{Methods}

\section{Sediment Sampling}

The Gregg In Situ Rhino Rig was used with either the CPT wireline sampling system or the Gregg In Situ Macro Core system. Applied Research Associates (ARA) developed the CPT wireline system with funding from the Department of Energy's National Energy Technology Laboratory (NETL). The wireline sampling tool uses 2.25-inch diameter rods with a removable dummy push tip and a one foot long core barrel with a locking mechanism that fits inside the push rods. The dummy tip is removed and core barrel deployed via the wire line at the desired sample collection depth. The rod string is advanced one foot forcing sediments into the core barrel. The core barrel is then brought to the surface and dummy tip reinserted via the wire line. With the dummy tip in place, the rod string is advanced to the next sample collection depth. The wireline tool allows the recovery of multiple soil samples without removing and reinserting the push rod string, significantly reducing the amount of time required to collect sediment samples.

The Macro Core system uses a 4-foot long core barrel at the distal end of the penetration rod string with a displaceable tip and plastic core collection tube inserted into the core barrel. The core barrel is pushed to the sample collection depth and the tip unlocking mechanism is actuated. The rods and core barrel are then advanced up to 4 feet, forcing the tip and sediments into the core collection tube. The rod string is then removed from the borehole and the core is collected at the surface. The core barrel and tip are replaced and the string is advanced to the next depth.

After the core was brought to the surface, a 2 cubic centimeter (cc) sediment plug sample was collected using a modified plastic syringe. The plug was immediately transferred to a 22-ml glass headspace vial with $5 \mathrm{ml}$ of nano-pure water. The vial was then sealed with a crimped Teflon-lined septum top for headspace analysis. Duplicate samples were collected at each depth.

\section{Sediment Headspace Analysis for VOCs}

The technique used to prepare and analyze sediment samples for VOC analysis is a modified version of EPA Method 5021 which has been used successfully at the SRS since 1991. A more detailed description of this method is provided in Appendix A. Each sample is weighed and then analyzed on the HP 5890 Series II or HP 6890 gas chromatograph (GC) using an automated head space sampler at $70^{\circ} \mathrm{C}$ for equivalent water concentrations. The GC is equipped with an electron capture and flame ionization detector connected in parallel. The column is a Supelco - VOCOL ${ }^{\mathrm{TM}}$ megabore borosilicate glass ( $60 \mathrm{~m} \times 0.76 \mathrm{~mm}$ ID $\times 1.5 \mu \mathrm{m}$ film thickness) specifically developed for volatile priority pollutants (EPA Methods 502, 602, and 8240). Mass soil concentrations (ppmm, $\mathrm{mg} / \mathrm{kg}$ ) are calculated based on an equal head space volume from $7.5 \mathrm{ml}$ of water standards and nominal $7 \mathrm{ml}$ of water/soil matrix, and are corrected for the mass difference between the soil and water. The gas chromatograph is calibrated using purchased certified mixtures in methanol that are 
diluted in deionized water to specific concentrations. Two reagent blanks of pure deionized water are included after the high concentration standards to ensure the transfer lines and column are being adequately flushed of residual solvents. The standard concentrations used for each head space sample run are: $3,5,10,50,100$, 1,000 , and $10,000 \mathrm{ppb}(\mu \mathrm{g} / \mathrm{l})$. A selected suite of compounds was chosen based on the primary contaminants expected at the A-014 outfall. The samples were analyzed for 1,1-dichloroethylene (1,1-DCE), carbon tetrachloride $\left(\mathrm{CCl}_{4}\right)$, cis-1,2dichloroethylene (cis-DCE), trichlorofluoromethane (Freon 11), chloroform, Freon 113, perchloroethylene (PCE), 1,1,1-trichloroethane (TCA), trichloroethylene (TCE), trans-1,2-dichloroethylene (trans-DCE), and toluene. The minimum detection limit (MDL) and minimum quantitation limit (MQL) for soil analysis is provided in Table 1.

The data reported from these analyses are considered screening level data.

Although standard laboratory methods are followed, the laboratory is not certified by any federal or state agency for analyses required for specific permit analysis requirements. All reported data should be accompanied with this flag.

Table 1 - Sediment Analysis Detection Limits

\begin{tabular}{|l|r|r|r|}
\hline Compound & $\begin{array}{c}\text { MDL } \\
(\mathbf{m g} / \mathbf{k g})\end{array}$ & $\begin{array}{c}\text { MQL } \\
(\mathbf{m g} / \mathbf{k g})\end{array}$ & $\begin{array}{c}\text { MQL } \\
(\mathbf{u g} / \mathbf{k g})\end{array}$ \\
\hline $1,1 \mathrm{DCE}$ & 0.002 & 0.003 & 3 \\
\hline CCl & 0.0001 & 0.003 & 3 \\
\hline cis-DCE & 0.04 & 0.12 & 124 \\
\hline Freon-11 & 0.0001 & 0.003 & 3 \\
\hline Chloroform & 0.0007 & 0.003 & 3 \\
\hline Freon-113 & 0.0003 & 0.003 & 3 \\
\hline PCE & 0.0001 & 0.003 & 3 \\
\hline TCA & 0.0003 & 0.003 & 3 \\
\hline TCE & 0.0004 & 0.003 & 3 \\
\hline Trans-DCE & 0.02 & 0.05 & 49 \\
\hline Toluene & 0.004 & 0.013 & 13 \\
\hline
\end{tabular}

\section{Soil Moisture Determination}

Sediment samples were collected in pre-weighed vials for soil moisture measurements. The samples were weighed and dried at $105 \mathrm{C}$ until the dry weight was stable. This measurement provides soil moisture by weight and follows ASTM method D2216. 


\section{Results and Contaminant Distribution}

A total of 378 one foot long cores at five locations were collected from which two sediment samples were analyzed (sample and duplicate). Concentrations indicating the presence of PCE NAPL occurred in 59 and TCE NAPL in 1 of the 378 samples collected. Figure 1 provides a three dimensional image created by interpolating the results of the sediment analyses. The image was created using Rockware software and was not well bound on the north and east sides, nevertheless it indicates the large area of shallow DNAPL contamination and lobes of DNAPL deeper in the vadose zone. DNAPL is present when PCE concentrations are above approximately $60 \mathrm{mg} / \mathrm{kg}$ and is shown on the image by the yellow contour. This volume of sediments is approximately $7000 \mathrm{ft}^{3}$ but only accounts for the imaged portion of the plume. This volume will be larger once the north and east sides of the plume are characterized. The coordinates for the sediment borings are provided in Table 2 and the results from the soil moisture tests are provided in Table 3 and Table 4. Plots of the distribution of PCE with respect to elevation at the five sediment sampling locations are shown in Figures 2-6. The sediment analysis results are provided in Appendix B.

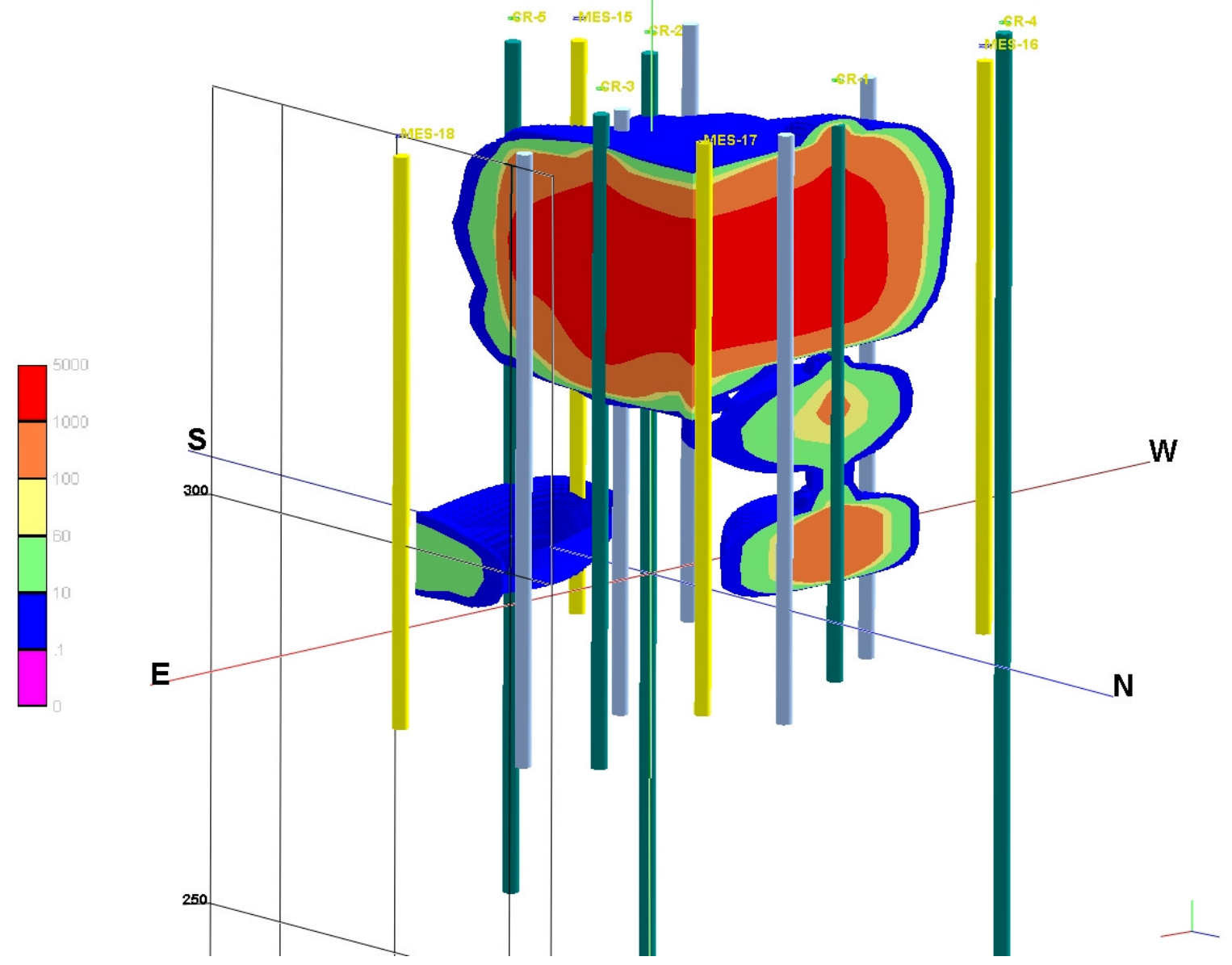

Figure 1 - PCE Sediment Concentration Plume above $10 \mathrm{mg} / \mathrm{kg}$ at the A-14 Outfall. The green cylinders are the sediment boring locations, the yellow and blue cylinders are the complex resistivity electrode string locations. 
Table 2 - SRS Coordinates for the Sediment Borings

\begin{tabular}{|c|c|c|c|}
\hline Boring & Easting & Northing & Elevation \\
\hline CR1 & 49950.46 & 102047.9 & 344.53 \\
\hline CR2 & 49950.71 & 102031.8 & 347.41 \\
\hline CR3 & 49962.19 & 102040 & 346.86 \\
\hline CR4 & 49933.54 & 102044.2 & 348.73 \\
\hline CR5 & 49954.43 & 102023.9 & 347.13 \\
\hline
\end{tabular}

Table 3 - Soil Moisture Results for Boring CR1

\begin{tabular}{|c|c|c|c|}
\hline Boring & $\begin{array}{c}\text { Depth } \\
\text { (ft) }\end{array}$ & $\begin{array}{c}\text { Sample } \\
\text { Date }\end{array}$ & $\begin{array}{c}\text { Moisture } \\
\text { (\% by wt) }\end{array}$ \\
\hline CR1 & 3 & $5 / 14 / 03$ & 17.3 \\
\hline CR1 & 5 & $5 / 14 / 03$ & 16.5 \\
\hline CR1 & 10 & $5 / 14 / 03$ & 16.8 \\
\hline CR1 & 15 & $5 / 14 / 03$ & 18.7 \\
\hline CR1 & 20 & $5 / 14 / 03$ & 11.4 \\
\hline CR1 & 30 & $5 / 14 / 03$ & 9.7 \\
\hline CR1 & 35 & $5 / 14 / 03$ & 18.5 \\
\hline CR1 & 40 & $5 / 14 / 03$ & 9.0 \\
\hline CR1 & 45 & $5 / 14 / 03$ & 12.6 \\
\hline CR1 & 50 & $5 / 14 / 03$ & 13.1 \\
\hline CR1 & 55 & $5 / 14 / 03$ & 8.5 \\
\hline CR1 & 60 & $5 / 14 / 03$ & 6.2 \\
\hline CR1 & 65 & $5 / 14 / 03$ & 6.8 \\
\hline
\end{tabular}


Table 4 - Soil Moisture Results for Boring CR2

\begin{tabular}{|c|c|c|c|}
\hline Boring & $\begin{array}{c}\text { Depth } \\
\text { (ft) }\end{array}$ & $\begin{array}{c}\text { Sample } \\
\text { Date }\end{array}$ & $\begin{array}{c}\text { Moisture } \\
\text { (\% by wt) }\end{array}$ \\
\hline CR2 & 1 & $5 / 19 / 03$ & 14.1 \\
\hline CR2 & 2 & $5 / 19 / 03$ & 12.5 \\
\hline CR2 & 3 & $5 / 19 / 03$ & 6.9 \\
\hline CR2 & 4 & $5 / 19 / 03$ & 17.3 \\
\hline CR2 & 5 & $5 / 19 / 03$ & 20.5 \\
\hline CR2 & 6 & $5 / 19 / 03$ & 22.6 \\
\hline CR2 & 7 & $5 / 19 / 03$ & 20.9 \\
\hline CR2 & 8 & $5 / 19 / 03$ & 18.5 \\
\hline CR2 & 9 & $5 / 19 / 03$ & 18.0 \\
\hline CR2 & 10 & $5 / 19 / 03$ & 17.3 \\
\hline CR2 & 11 & $5 / 19 / 03$ & 17.6 \\
\hline CR2 & 12 & $5 / 19 / 03$ & 16.0 \\
\hline CR2 & 13 & $5 / 19 / 03$ & 16.6 \\
\hline CR2 & 14 & $5 / 19 / 03$ & 16.4 \\
\hline CR2 & 15 & $5 / 19 / 03$ & 17.2 \\
\hline CR2 & 16 & $5 / 19 / 03$ & 14.4 \\
\hline CR2 & 17 & $5 / 19 / 03$ & 13.1 \\
\hline CR2 & 18 & $5 / 19 / 03$ & 14.0 \\
\hline CR2 & 19 & $5 / 19 / 03$ & 15.1 \\
\hline CR2 & 20 & $5 / 19 / 03$ & 12.6 \\
\hline CR2 & 21 & $5 / 19 / 03$ & 11.4 \\
\hline CR2 & 22 & $5 / 19 / 03$ & 11.0 \\
\hline CR2 & 23 & $5 / 19 / 03$ & 10.2 \\
\hline CR2 & 24 & $5 / 19 / 03$ & 12.9 \\
\hline CR2 & 25 & $5 / 19 / 03$ & 11.1 \\
\hline & & \multicolumn{3}{|c}{} \\
\hline
\end{tabular}

\section{Discussion of NAPL Presence Evaluation}

The chemical analyses of sediment samples measure the total mass of contaminant present per unit dry weight of soil sample. These analyses do not distinguish between contaminant mass sorbed to the soil, dissolved in the soil water, volatilized in soil gas, or present as a separate phase (NAPL). The soil concentration where separate phase tetracholoroethylene (PCE), and trichloroethylene (TCE), must be present in the samples collected at the A-014 outfall was determined using the method of Feenstra et al (1991) based on equilibrium partitioning theory as described in Cohen and Mercer (Cohen and Mercer 1993). NAPL can be inferred to be present when the soil concentrations exceed the theoretical maximum chemical mass that can be adsorbed to soil, dissolved in soil water, and volatilized in soil gas. For saturated soils, an apparent soil water concentration $\left(\mathrm{C}_{\text {asw }}\right)$ is calculated from the total soil concentrations by assuming equilibrium partitioning (Feenstra et al. 1991). 
For saturated zone samples,

$$
\mathrm{C}_{\mathrm{asw}}=\left(\mathrm{X}_{\mathrm{T}} \rho_{\mathrm{b}}\right) /\left(\mathrm{K}_{\mathrm{d}} \rho_{\mathrm{b}}+\phi\right),
$$

where $\mathrm{X}_{\mathrm{T}}$ is the total soil mass fraction, $\mathrm{K}_{\mathrm{d}}$ is a linear sorption coefficient, $\rho_{b}$ is soil dry bulk density, and $\phi$ is the porosity.

For unsaturated samples,

$$
\mathrm{C}_{\mathrm{w}}=\left(\mathrm{X}_{\mathrm{T}} \rho_{\mathrm{b}}\right) /\left(\mathrm{K}_{\mathrm{d}} \rho_{\mathrm{b}}+\phi \mathrm{S}_{\mathrm{w}}+\phi \mathrm{S}_{\mathrm{g}} \mathrm{H}\right),
$$

where $\mathrm{C}_{\mathrm{w}}$ is the apparent soil water concentration for unsaturated conditions, $\mathrm{S}_{\mathrm{w}}$ and $\mathrm{S}_{g}$ are the water and gas phase pore saturation values, and $\mathrm{H}$ is the Henry's Law partitioning coefficient for the compound at the appropriate temperature. Using this equilibrium relation, a threshold for the presence of NAPL in a soil sample can be determined by the measurement of mass fraction using the following equation:

$$
\mathrm{C}_{\mathrm{w}}{ }^{\text {sat }}<=\left(\mathrm{X}_{\mathrm{T}} \rho_{\mathrm{b}}\right) /\left(\mathrm{K}_{\mathrm{d}} \rho_{\mathrm{b}}+\phi \mathrm{S}_{\mathrm{w}}+\phi \mathrm{S}_{\mathrm{g}} \mathrm{H}\right),
$$

where $\mathrm{C}_{\mathrm{w}}{ }^{\text {sat }}$ is the aqueous solubility concentration of a compound.

These relationships were determined for the SRS sediments over the measured range of average fraction of organic carbon values ( $f_{o c}=0.0001$ to 0.001$)$ and a representative average porosity values $(\phi=0.4)$. Table 5 provides the minimum concentration for NAPL to be present in the vadose sediments at the A-014 outfall. These values provide the worse case scenario of the lowest fraction of organic carbon, water saturation, and porosity.

Table 5 - Theoretical Concentrations for the Presence of NAPL in the SRS Vadose Zone Sediments

\begin{tabular}{|c|c|}
\hline Compound & $\begin{array}{c}\text { Minimum Concentration } \\
\text { for presence of NAPL }\end{array}$ \\
\hline PCE & $61 \mathrm{mg} / \mathrm{kg}$ \\
\hline TCE & $320 \mathrm{mg} / \mathrm{kg}$ \\
\hline
\end{tabular}

\section{Recommendations}

Additional borings are recommended to delineate the edges of the DNAPL source area. These locations are to the north and east of the current borings. The northern boring will likely require angled drilling under $\mathrm{D}$ Road at the mouth of the outfall. An additional boring through the middle of the DNAPL plume and into the water table is recommended to evaluate the presence of DNAPL in the groundwater. 


\section{A-14 CR01}

E. 49950.46, N. 102047.88, surface el. 344.53

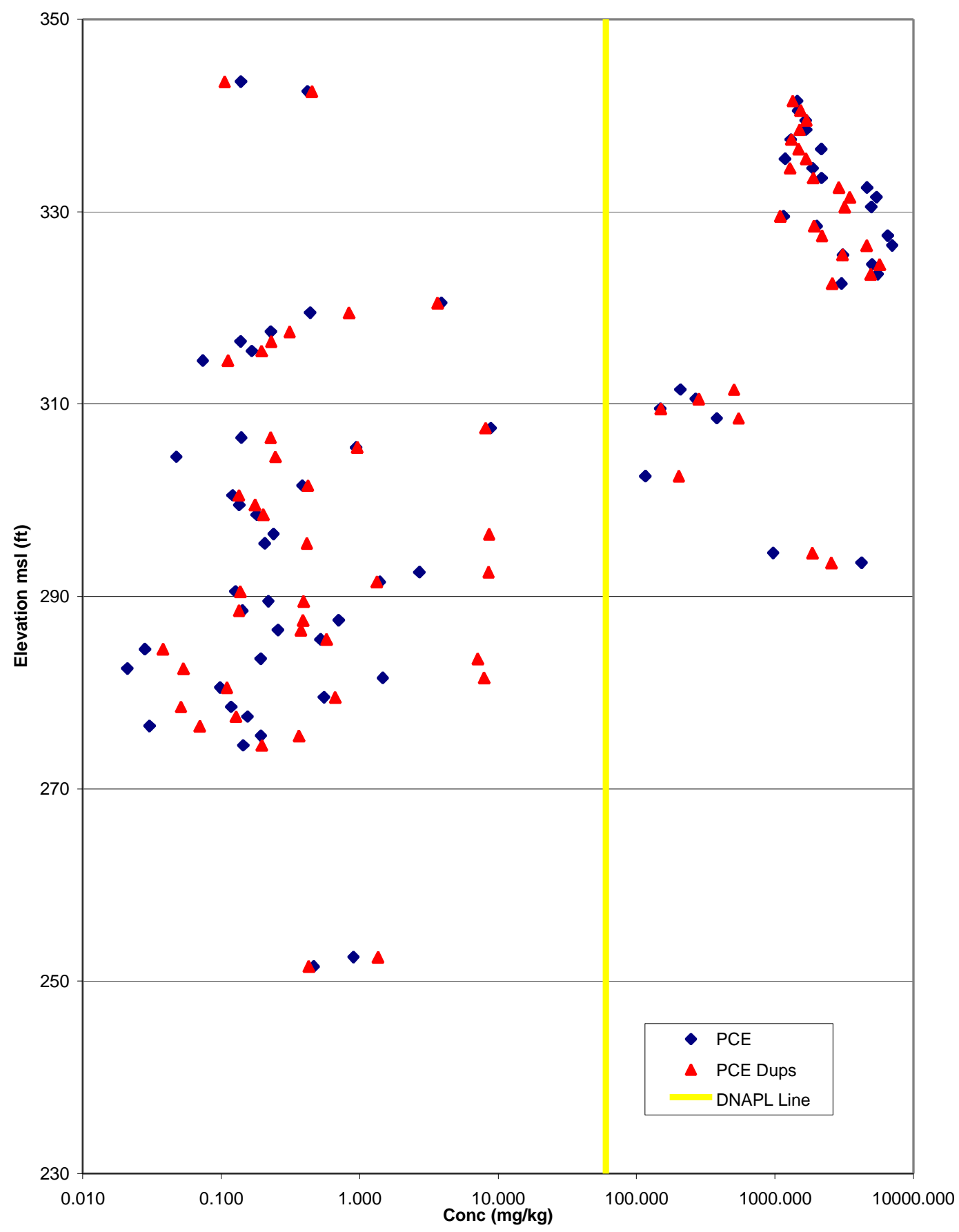

Figure 2 - CR01 PCE Results 


\section{A-14 CR02}

E. 49950.71 , N. 102031.83 , surface el. 347.41

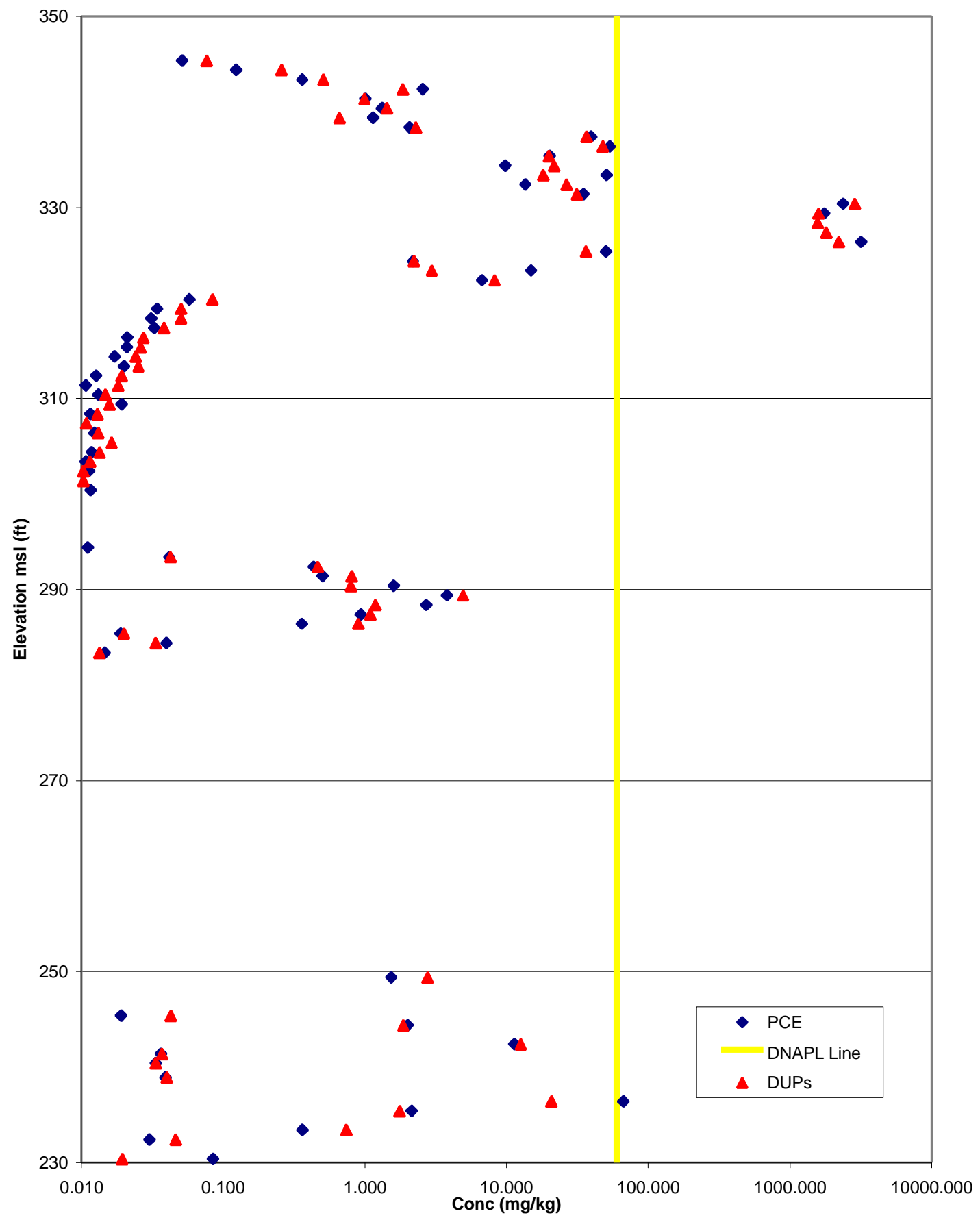

Figure 3 - CR02 PCE Results 


\section{A-14 CR03}

E. 49962.19, N. 102039.98, surface el. 346.86

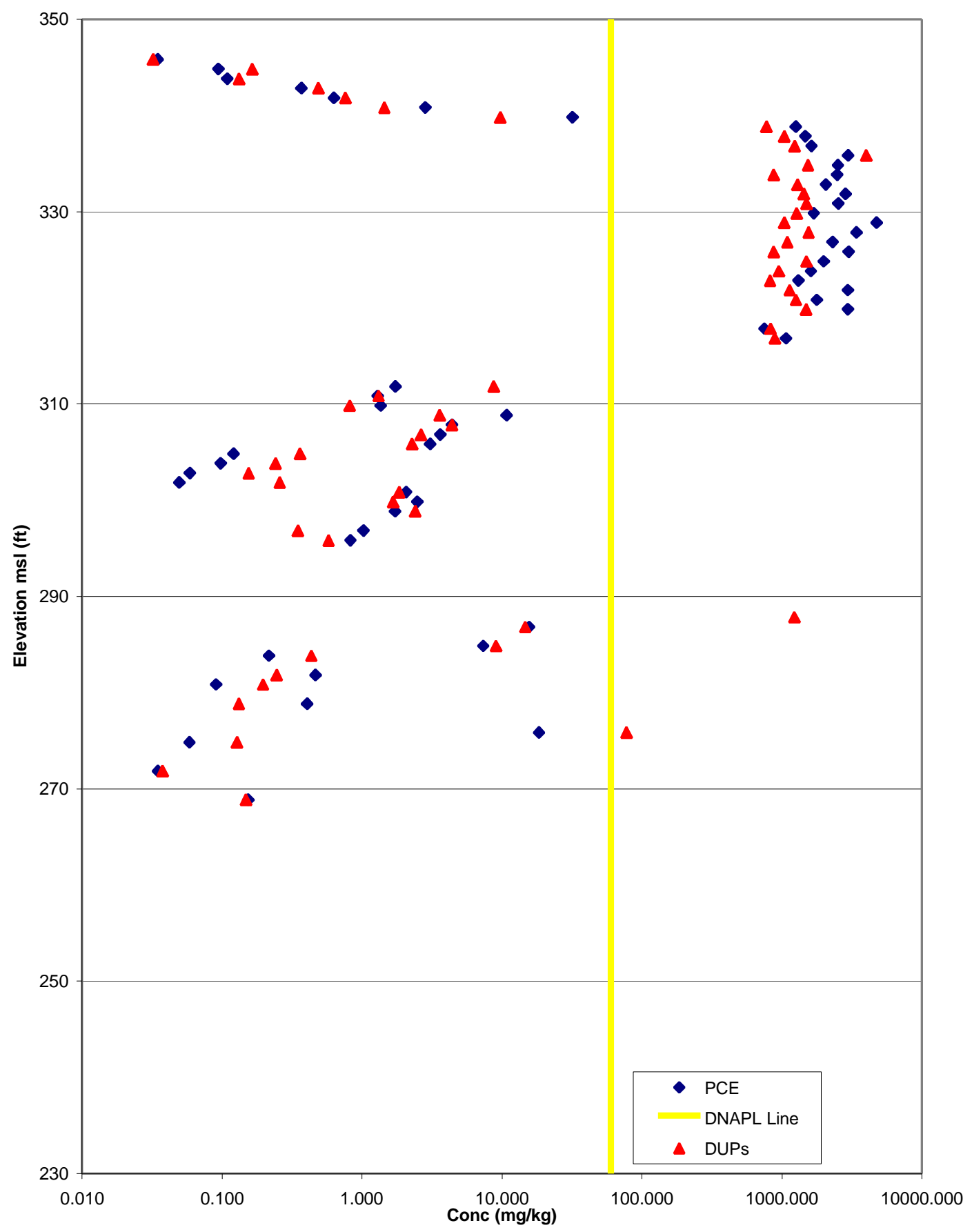

Figure 4 - CR03 PCE Results 


\section{A-14 CR04}

E. 49933.54, N. 102044.23, surface el. 348.73

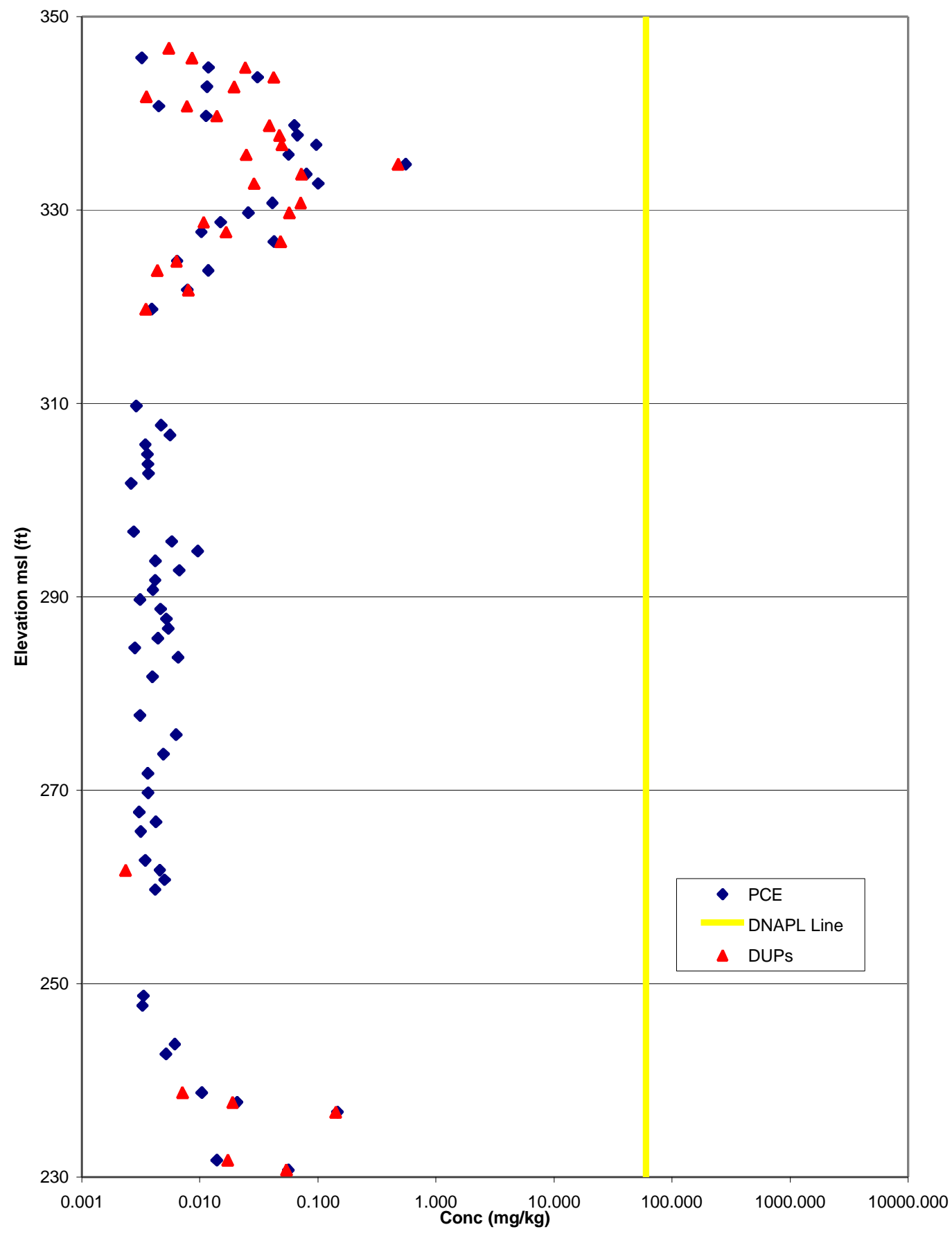

Figure 5 - CR04 PCE Results 


\section{A-14 CR05}

E. 49954.43, N. 102023.92, surface el. 347.13

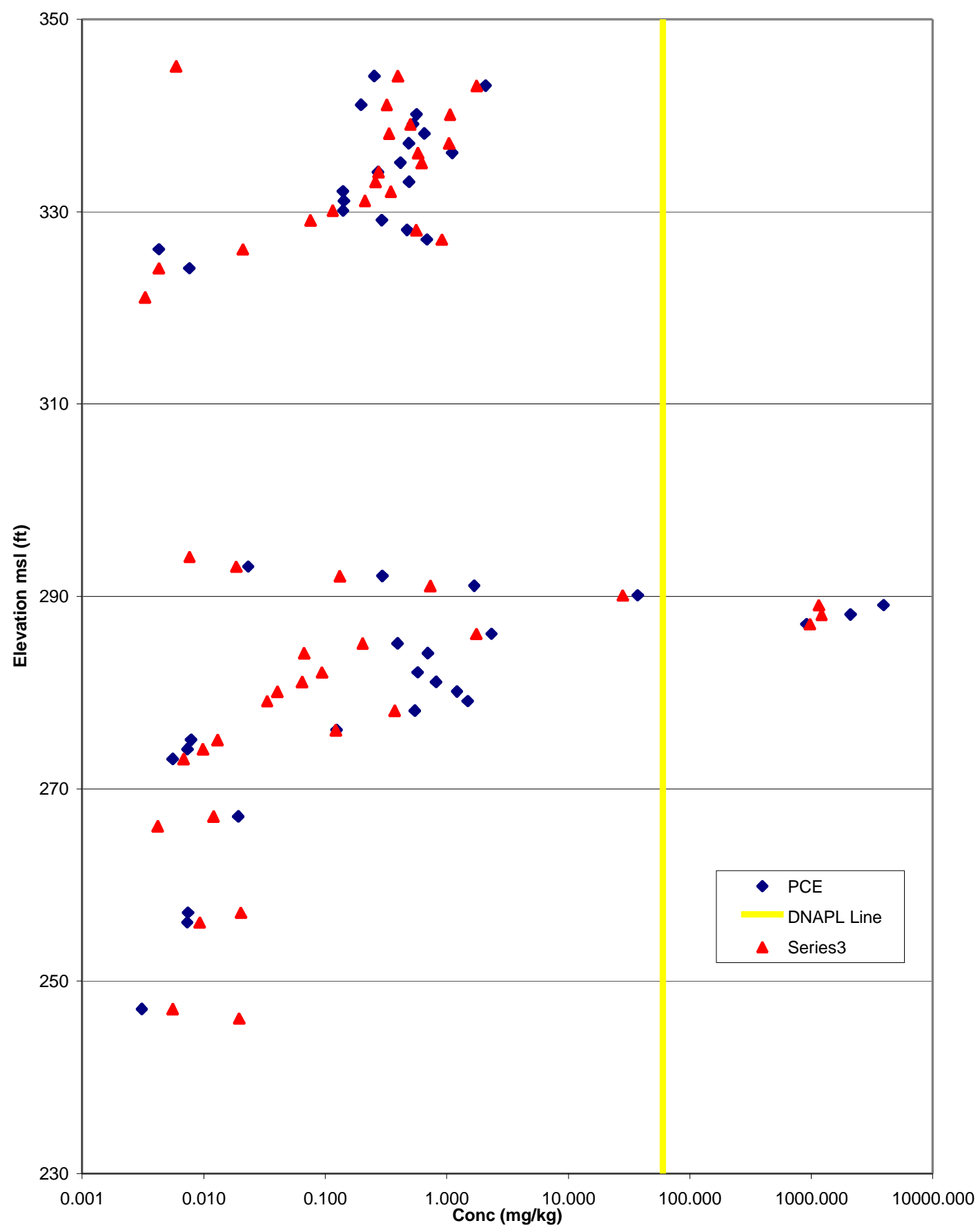

Figure 6 - CR05 PCE Results 


\section{References}

Cohen, R. M., and J. W. Mercer, 1993, DNAPL Site Evaluation: C. K. Smoley-CRC Press, Boca Raton, Florida

Feenstra, S. D.M. Mackay, and J.A. Cherry, 1991. A method for assessing residual NAPL based on organic chemical concentrations in soil samples: Groundwater Monitoring Review, 11(2):128-136. 


\section{Appendix A - Heated Headspace Gas Chromatography Phase Conversion}

Using the heated headspace method, the concentration measured by the gas chromatograph is assumed to include the total mass of volatile chemical analyte in the sample. The $22 \mathrm{ml}$ glass headspace vials, containing approximately $2 \mathrm{cc}$ of sediment sample and $5 \mathrm{ml}$ of added deionized water and sealed by teflon septum and crimp cap, are heated to a temperature of $70^{\circ} \mathrm{C}$ and maintained at that temperature for at least 20 minutes. At this temperature, the entire mass of chemical is assumed to have been transported into the gas phase in the headspace of the vial from all other phases (aqueous, sorbed, liquid). The value measured in the gas phase is output as an aqueous concentration (because it is correlated to the prepared aqueous standards using a volume of $7.5 \mathrm{ml}$ of water in a head space vial). It is then converted to a mass fraction of chemical in the saturated soil sample based on the measured mass of sediment. The measured sample mass is determined by weighing the entire headspace vial and subtracting out the average mass of the vial plus $5 \mathrm{ml}$ of water (added at the time of soil sampling). The equation describing this conversion is as follows:

$$
X_{T}(m g / k g)=\frac{C_{c s t d}(m g / l) * V_{s t d}(m l)}{M_{s}(g)} * \frac{\left(\frac{1(l)}{1000(m l)}\right)}{\left(\frac{1(k g)}{1000(g)}\right)},
$$

where $\boldsymbol{X}_{T}$ is mass fraction of chemical in the sediment sample, $\boldsymbol{C}_{c s t d}$ is the measured concentration of the sample based on a correlation to prepared aqueous standards, $\boldsymbol{V}_{\boldsymbol{s t d}}$ is the volume of water in the aqueous standards $(7.5 \mathrm{ml})$ and $\boldsymbol{M}_{\boldsymbol{s}}$ is the mass of the sediment sample. The mass of the sediment sample is calculated by the following equation:

$$
M_{s}=M_{T}-M_{\text {vavg }}-M_{a w},
$$

where $\boldsymbol{M}_{\boldsymbol{T}}$ is the total mass of the sealed sample in the headspace vial, $\boldsymbol{M}_{\boldsymbol{v a v g}}$ is the average mass of $22 \mathrm{ml}$ headspace vials and septum crimp caps, and $\boldsymbol{M}_{a w}$ is the mass of added water (usually $5 \mathrm{ml}$ ). The value of $\boldsymbol{X}_{\boldsymbol{T}}$ is a comprehensive representation of the mass of chemical in the sediment sample as it is collected. 


\section{Appendix B - CR Boring Series Analytical Results Tables}

Table 6 - Sediment Analysis Results for Boring CR01

\begin{tabular}{|c|c|c|c|c|c|c|c|c|c|c|}
\hline Sample ID & Boring & Depth (ft) & Type & $\begin{array}{c}\text { Date } \\
\text { Collected }\end{array}$ & $\begin{array}{c}\text { Date } \\
\text { Analyzed }\end{array}$ & $\begin{array}{c}\text { PCE } \\
(\mathrm{mg} / \mathrm{kg})\end{array}$ & $\begin{array}{c}\text { TCE } \\
(\mathrm{mg} / \mathrm{kg})\end{array}$ & $\begin{array}{l}\text { C-DCE } \\
\text { (mg/kg) }\end{array}$ & $\begin{array}{c}\text { TCA } \\
(\mathrm{mg} / \mathrm{kg})\end{array}$ & $\begin{array}{l}\text { 11DCE } \\
(\mathrm{mg} / \mathrm{kg})\end{array}$ \\
\hline A14-CR01-01 & CR01 & 1 & sample & $5 / 14 / 2003$ & $5 / 15 / 2003$ & 0.139 & 0.002 & 0.000 & 0.000 & 0.000 \\
\hline A14-CR01-01 & CR01 & 1 & duplicate & $5 / 14 / 2003$ & $5 / 16 / 2003$ & 0.106 & 0.003 & 0.000 & 0.000 & $\mathrm{~nm}$ \\
\hline A14-CR01-02 & R01 & 2 & sample & $5 / 14 / 2003$ & $5 / 15 / 2003$ & 0.420 & 0.005 & 0.000 & 0.000 & 0.000 \\
\hline A14-CR01-02 & R01 & 2 & duplicate & $5 / 14 / 2003$ & $5 / 16 / 2003$ & 0.452 & & 0.031 & 0.000 & $\mathrm{~nm}$ \\
\hline A14-CR01-03 & CR01 & 3 & sample & $5 / 14 / 2003$ & $5 / 15 / 2003$ & 1445.796 & 22.869 & 0.187 & 0.076 & 0.007 \\
\hline $\mathrm{A} 14-\mathrm{C}$ & CR01 & 3 & duplicate & $5 / 14 / 2003$ & $5 / 16 / 2003$ & 1344.232 & 34.701 & 0.573 & 0.213 & $\mathrm{~nm}$ \\
\hline A14-CR01-04 & CR01 & 4 & sample & $5 / 14 / 2003$ & $5 / 15 / 2003$ & 1475.379 & 24.871 & 0.205 & 0.064 & 0.005 \\
\hline A14-CR01-04 & CR01 & 4 & duplicate & $5 / 14 / 2003$ & $5 / 16 / 2003$ & 1535.840 & 28.709 & 0.192 & 0.125 & $\mathrm{~nm}$ \\
\hline A14-CF & $R 01$ & 5 & sample & $5 / 14 / 2003$ & $5 / 15 / 2003$ & 1675.446 & 22.935 & 0.108 & 0.040 & 0.008 \\
\hline A14-C & R01 & 5 & duplicate & $5 / 14 / 2003$ & $5 / 16 / 2003$ & 1693.686 & 39.921 & 0.379 & 0.147 & $\mathrm{~nm}$ \\
\hline A14-CR01-06 & CR01 & 6 & sample & $5 / 14 / 2003$ & $5 / 15 / 2003$ & 1678.540 & 35.407 & 0.187 & 0.032 & 0.004 \\
\hline A14-CR01-06 & CR01 & 6 & duplicate & $5 / 14 / 2003$ & $5 / 16 / 2003$ & 1512.563 & 38.396 & 0.303 & 0.057 & $\mathrm{~nm}$ \\
\hline A14-CR & $R 01$ & 7 & sample & $5 / 14 / 2003$ & $5 / 15 / 2003$ & 1308.503 & 24.776 & 0.218 & 0.020 & 0.000 \\
\hline A14-C & & 7 & duplic & $5 / 14 / 2003$ & $5 / 16 / 2003$ & 1319.233 & 37.342 & 0.179 & 0.054 & $\mathrm{~nm}$ \\
\hline A14- & CR01 & 8 & sample & $5 / 14 / 2003$ & $5 / 15 / 2003$ & 2163.288 & 61.172 & 0.239 & 0.059 & 0.007 \\
\hline A14-C & & 8 & duplicate & $5 / 14 / 2003$ & $5 / 16 / 2003$ & 1487.120 & 53.429 & 0.406 & 0.084 & $\mathrm{~nm}$ \\
\hline A14-C & CR01 & 9 & sample & $5 / 14 / 2003$ & $5 / 15 / 2003$ & 1183.482 & 28.633 & 0.341 & 0.018 & 0.018 \\
\hline A14-C & & 9 & duplic & $5 / 14 / 2003$ & $5 / 16 / 2003$ & 1683.251 & 80.431 & 1.215 & 0.112 & $\mathrm{~nm}$ \\
\hline A14-C & & 10 & sample & $5 / 14 / 2003$ & $5 / 15 /$ & 1882.934 & 71.281 & 0.470 & 0.040 & 0.010 \\
\hline $\mathrm{A} 14-\mathrm{C}$ & CR01 & 10 & duplicate & $5 / 14 / 2003$ & $5 / 16 / 2003$ & 1288.453 & 46.688 & 0.601 & 0.044 & $\mathrm{~nm}$ \\
\hline A14-C & CR01 & 11 & sample & $5 / 14 / 2003$ & $5 / 15 / 2003$ & 2178.189 & 78.240 & 0.468 & 0.042 & 0.009 \\
\hline A14-C & CR01 & 11 & duplicate & $5 / 14 / 2003$ & $5 / 16 / 2003$ & 1897.134 & 81.248 & 0.644 & 0.065 & $\mathrm{~nm}$ \\
\hline A14-C & & 12 & sample & $5 / 14 / 2003$ & $5 / 15 / 20$ & 4633.237 & 124.116 & 0.519 & 0.055 & 0.020 \\
\hline & & 12 & duplicate & $5 / 14 / 2003$ & & 2905.308 & 111.268 & 1.026 & 0.090 & $\mathrm{~nm}$ \\
\hline A14-CR & & 13 & sample & $5 / 14 / 2003$ & $5 / 15 / 2003$ & 5431.841 & 154.531 & 0.642 & 0.068 & 0.020 \\
\hline A14-CR01-13 & CR01 & 13 & duplicate & $5 / 14 / 2003$ & $5 / 16 / 2003$ & 3475.708 & 124.702 & 1.150 & 0.095 & $\mathrm{~nm}$ \\
\hline
\end{tabular}




\begin{tabular}{|c|c|c|c|c|c|c|c|c|c|c|}
\hline Sample ID & Boring & Depth (ft) & Type & $\begin{array}{c}\text { Date } \\
\text { Collected }\end{array}$ & $\begin{array}{c}\text { Date } \\
\text { Analyzed }\end{array}$ & $\begin{array}{c}\text { PCE } \\
(\mathrm{mg} / \mathrm{kg})\end{array}$ & $\begin{array}{c}\text { TCE } \\
(\mathrm{mg} / \mathrm{kg})\end{array}$ & $\begin{array}{l}\text { C-DCE } \\
(\mathrm{mg} / \mathrm{kg})\end{array}$ & $\begin{array}{c}\text { TCA } \\
(\mathrm{mg} / \mathrm{kg})\end{array}$ & $\begin{array}{r}\text { 11DCE } \\
(\mathrm{mg} / \mathrm{kg})\end{array}$ \\
\hline A14-CR01-14 & CR01 & 14 & sample & $5 / 15 / 2003$ & $5 / 15 / 2003$ & 4976.042 & 133.930 & 0.644 & 0.052 & 0.021 \\
\hline A14-CR01-14 & CR01 & 14 & duplicate & $5 / 15 / 2003$ & $5 / 16 / 2003$ & 3190.689 & 123.844 & 1.528 & 0.086 & $\mathrm{~nm}$ \\
\hline A14-CR01-15 & CR01 & 15 & sample & $5 / 15 / 2003$ & $5 / 15 / 2003$ & 1160.780 & 40.670 & 0.280 & 0.017 & 0.013 \\
\hline A14-CR01-15 & CR01 & 15 & duplicate & $5 / 15 / 2003$ & $5 / 16 / 2003$ & 1094.781 & 47.803 & 0.583 & 0.032 & $\mathrm{~nm}$ \\
\hline A14-CR01-16 & CR01 & 16 & sample & $5 / 15 / 2003$ & $5 / 15 / 2003$ & 2012.699 & 72.775 & 0.520 & 0.028 & 0.000 \\
\hline A14-CR01-16 & CR01 & 16 & duplicate & $5 / 15 / 2003$ & $5 / 16 / 2003$ & 1925.203 & 66.518 & 0.444 & 0.041 & $\mathrm{~nm}$ \\
\hline A14-CR01-17 & CR01 & 17 & sample & $5 / 15 / 2003$ & $5 / 15 / 2003$ & 6537.106 & 138.909 & 0.405 & 0.044 & 0.000 \\
\hline A14-CR01-17 & CR01 & 17 & duplicate & $5 / 15 / 2003$ & $5 / 16 / 2003$ & 2187.361 & 81.974 & 0.636 & 0.050 & $\mathrm{~nm}$ \\
\hline A14-CR01-18 & CR01 & 18 & sample & $5 / 15 / 2003$ & $5 / 15 / 2003$ & 7051.819 & 174.519 & 0.404 & .058 & 0.000 \\
\hline A14-CR0 & CR01 & 18 & duplicate & $5 / 15 / 2003$ & $5 / 16 / 2003$ & 4602.929 & 141.695 & 0.839 & 0.083 & $\mathrm{~nm}$ \\
\hline A14-CR01-19 & CR01 & 19 & sample & $5 / 15 / 2003$ & $5 / 15 / 2003$ & 3093.694 & 93.008 & 0.154 & 0.028 & 0.000 \\
\hline A14-CR01-19 & CR01 & 19 & duplicate & $5 / 15 / 2003$ & $5 / 16 / 2003$ & 3086.120 & 91.082 & 0.315 & 0.042 & $\mathrm{~nm}$ \\
\hline A14-CR01-20 & CR01 & 20 & sam & $5 / 15 / 2003$ & $5 / 15 / 2003$ & 5053.880 & 114.516 & 0.000 & 0.021 & 0.000 \\
\hline A14- & CR01 & 20 & duplic & $5 / 15 / 2003$ & $5 / 16 / 2003$ & 5726.317 & 148.089 & 0.241 & 0.048 & $\mathrm{~nm}$ \\
\hline A14-CR01-21 & CR01 & 21 & sample & $5 / 15 / 2003$ & $5 / 15 / 2003$ & 5536.200 & 60.567 & 0.128 & 0.006 & 0.000 \\
\hline A14-CR01-21 & CR01 & 21 & duplicate & $5 / 15 / 2003$ & $5 / 16 / 2003$ & 4948.678 & 44.517 & 0.645 & 0.007 & $\mathrm{~nm}$ \\
\hline A14-CR01-22 & R01 & 22 & sam & $5 / 15 / 2003$ & $5 / 15 / 2003$ & 3037.277 & 7.117 & 0.000 & 0.000 & 0.000 \\
\hline A14-CR01-22 & CR01 & 22 & duplicate & $5 / 15 / 2003$ & $5 / 16 / 2003$ & 2595.208 & 8.861 & 0.506 & 0.000 & $\mathrm{~nm}$ \\
\hline A14-CR01-24 & CR01 & 24 & sample & $5 / 15 / 2003$ & $5 / 15 / 2003$ & \begin{tabular}{|l|}
3.898 \\
\end{tabular} & 0.022 & 0.000 & 0.000 & 0.000 \\
\hline A14-CR01-24 & CR01 & 24 & duplic & $5 / 15 / 2003$ & $5 / 16 / 2003$ & 3.651 & 0.080 & 0.000 & 0.000 & $\mathrm{~nm}$ \\
\hline A14-CR01-25 & CR01 & 25 & sample & $5 / 15 / 2003$ & $5 / 15 / 2003$ & 0.441 & 0.007 & 0.000 & 0.000 & 0.000 \\
\hline A14-CR01-25 & R01 & 25 & duplicate & $5 / 15 / 2003$ & $5 / 16 / 2003$ & 0.837 & 0.029 & 0.000 & 0.000 & $\mathrm{~nm}$ \\
\hline A14- & CR01 & 27 & sam & $5 / 15 / 2003$ & $5 / 15 / 2003$ & 0.229 & 0.000 & 0.000 & 0.000 & 0.000 \\
\hline A14-CR01-27 & CR01 & 27 & duplicate & $5 / 15 / 2003$ & $5 / 16 / 2003$ & 0.313 & 0.011 & 0.000 & 0.000 & $\mathrm{~nm}$ \\
\hline A14-CR01-28 & CR01 & 28 & sample & $5 / 15 / 2003$ & $5 / 15 / 2003$ & 0.138 & 0.000 & 0.000 & 0.000 & 0.000 \\
\hline A14-CR01-28 & CR01 & 28 & duplicate & $5 / 15 / 2003$ & $5 / 16 / 2003$ & 0.229 & 0.008 & 0.000 & 0.000 & $\mathrm{~nm}$ \\
\hline A14-C & $\mathrm{R} 01$ & 29 & sam & $5 / 15 / 2003$ & $5 / 16 / 2003$ & 0.167 & 0.004 & 0.000 & 0.000 & 0.000 \\
\hline A14-CR01-29 & CR01 & 29 & duplicate & $5 / 15 / 2003$ & $5 / 19 / 2003$ & 0.196 & 0.006 & 0.000 & 0.000 & $\mathrm{~nm}$ \\
\hline A14-CR01-30 & CR01 & 30 & sample & $5 / 15 / 2003$ & $5 / 16 / 2003$ & 0.074 & 0.000 & 0.000 & 0.000 & 0.000 \\
\hline A14-CR01-30 & CR01 & 30 & duplicate & $5 / 15 / 2003$ & $5 / 19 / 2003$ & 0.112 & 0.003 & 0.000 & 0.000 & $\mathrm{~nm}$ \\
\hline A14-CR01-33 & CR01 & 33 & sample & $5 / 15 / 2003$ & $5 / 16 / 2003$ & 207.455 & 2.100 & 0.000 & 0.000 & 0.018 \\
\hline
\end{tabular}




\begin{tabular}{|c|c|c|c|c|c|c|c|c|c|c|}
\hline Sample ID & Boring & Depth (ft) & Type & $\begin{array}{c}\text { Date } \\
\text { Collected }\end{array}$ & $\begin{array}{c}\text { Date } \\
\text { Analyzed }\end{array}$ & $\begin{array}{c}\text { PCE } \\
(\mathrm{mg} / \mathrm{kg})\end{array}$ & $\begin{array}{c}\text { TCE } \\
(\mathrm{mg} / \mathrm{kg})\end{array}$ & $\begin{array}{l}\text { C-DCE } \\
(\mathrm{mg} / \mathrm{kg})\end{array}$ & $\begin{array}{c}\text { TCA } \\
(\mathrm{mg} / \mathrm{kg})\end{array}$ & $\begin{array}{r}\text { 11DCE } \\
(\mathrm{mg} / \mathrm{kg})\end{array}$ \\
\hline A14-CR01-33 & CR01 & 33 & duplicate & $5 / 15 / 2003$ & $5 / 19 / 2003$ & 507.265 & 6.912 & 0.117 & 0.009 & $\mathrm{~nm}$ \\
\hline A14-CR01-34 & CR01 & 34 & sample & $5 / 15 / 2003$ & $5 / 16 / 2003$ & 268.925 & 4.170 & 0.072 & 0.004 & 0.006 \\
\hline A14-CR01-34 & CR01 & 34 & duplicate & $5 / 15 / 2003$ & $5 / 19 / 2003$ & 283.049 & 4.157 & 0.150 & 0.005 & $\mathrm{~nm}$ \\
\hline A14-CR01-35 & CR01 & 35 & sample & $5 / 15 / 2003$ & $5 / 16 / 2003$ & 148.581 & 1.817 & 0.000 & 0.000 & 0.006 \\
\hline A14-CR01-35 & CR01 & 35 & duplicate & $5 / 15 / 2003$ & $5 / 19 / 2003$ & 150.276 & 1.926 & 0.084 & 0.002 & $\mathrm{~nm}$ \\
\hline A14-CR01-36 & CR01 & 36 & sample & $5 / 15 / 2003$ & $5 / 16 / 2003$ & 380.719 & 5.332 & 0.000 & 0.003 & 0.036 \\
\hline A14-CR01-36 & CR01 & 36 & duplicate & $5 / 15 / 2003$ & $5 / 19 / 2003$ & 549.551 & 10.699 & 0.119 & 0.009 & $\mathrm{~nm}$ \\
\hline A14-CR01-37 & CR01 & 37 & sample & $5 / 15 / 2003$ & $5 / 16 / 2003$ & 8.854 & 0.125 & 0.000 & 0.000 & 0.000 \\
\hline A14-CR01-37 & CR01 & 37 & duplicate & $5 / 15 / 2003$ & $5 / 19 / 2003$ & 8.138 & 0.125 & 0.000 & .000 & $\mathrm{~nm}$ \\
\hline A14-CR01-38 & CR01 & 38 & sample & $5 / 15 / 2003$ & $5 / 16 / 2003$ & 0.140 & 0.000 & 0.000 & 0.000 & 0.000 \\
\hline A14-CR01-38 & CR01 & 38 & duplicate & $5 / 15 / 2003$ & $5 / 19 / 2003$ & 0.228 & 0.005 & 0.000 & 0.000 & $\mathrm{~nm}$ \\
\hline A14-CR01-39 & CR01 & 39 & sample & $5 / 15 / 2003$ & $5 / 16 / 2003$ & 0.948 & 0.019 & 0.000 & 0.000 & 0.000 \\
\hline A14-CR01-39 & CR01 & 39 & duplicate & $5 / 15 / 2003$ & $5 / 19 / 2003$ & 0.962 & 0.036 & 0.000 & 0.000 & $\mathrm{~nm}$ \\
\hline A14- & CR01 & 40 & sample & $5 / 15 / 2003$ & $5 / 16 / 2003$ & 0.047 & 0.000 & 0.000 & 0.000 & 0.000 \\
\hline A14-CR01-40 & CR01 & 40 & duplicate & $5 / 15 / 2003$ & $5 / 19 / 2003$ & 0.247 & 0.006 & 0.000 & 0.000 & $\mathrm{~nm}$ \\
\hline A14-CR01-42 & CR01 & 42 & sample & $5 / 15 / 2003$ & $5 / 16 / 2003$ & 115.929 & 1.703 & 0.057 & 0.000 & 0.000 \\
\hline A14-CR01-42 & SR01 & 42 & duplicate & $5 / 15 / 2003$ & $5 / 19 / 2003$ & 202.852 & 2.439 & 0.068 & 0.003 & $\mathrm{~nm}$ \\
\hline A14-CR01-43 & CR01 & 43 & sample & $5 / 15 / 2003$ & $5 / 16 / 2003$ & 0.385 & 0.005 & 0.000 & 0.000 & 0.000 \\
\hline A14-CR01-43 & CR01 & 43 & duplicate & $5 / 15 / 2003$ & $5 / 19 / 2003$ & 0.424 & 0.010 & 0.000 & 0.000 & $\mathrm{~nm}$ \\
\hline A14-CR01-44 & CR01 & 44 & sample & $5 / 15 / 2003$ & $5 / 16 / 2003$ & 0.121 & 0.004 & 0.000 & 0.000 & 0.000 \\
\hline A14-CR01-44 & CR01 & 44 & duplicate & $5 / 15 / 2003$ & $5 / 19 / 2003$ & 0.134 & 0.004 & 0.000 & 0.000 & $\mathrm{~nm}$ \\
\hline A14-CR01-45 & R01 & 45 & sample & $5 / 15 / 2003$ & $5 / 16 / 2003$ & 0.135 & 0.000 & 0.000 & 0.000 & 0.000 \\
\hline A14-C & CR01 & 45 & duplicate & $5 / 15 / 2003$ & $5 / 19 / 2003$ & 0.175 & 0.000 & 0.000 & 0.000 & $\mathrm{~nm}$ \\
\hline A14-CR01-46 & CR01 & 46 & sample & $5 / 15 / 2003$ & $5 / 16 / 2003$ & 0.181 & 0.000 & 0.000 & 0.000 & 0.000 \\
\hline A14-CR01-46 & CR01 & 46 & duplicate & $5 / 15 / 2003$ & $5 / 19 / 2003$ & 0.202 & 0.003 & 0.000 & 0.000 & $\mathrm{~nm}$ \\
\hline A14-CR01-48 & CR01 & 48 & sample & $5 / 15 / 2003$ & $5 / 16 / 2003$ & 0.239 & 0.005 & 0.000 & 0.000 & 0.000 \\
\hline A14-CR01-48 & CR01 & 48 & duplicate & $5 / 15 / 2003$ & $5 / 19 / 2003$ & 8.624 & 0.098 & 0.000 & 0.000 & $\mathrm{~nm}$ \\
\hline A14-CR01-49 & CR01 & 49 & sample & $5 / 16 / 2003$ & $5 / 16 / 2003$ & 0.207 & 0.004 & 0.000 & 0.000 & 0.000 \\
\hline A14-CR01-49 & CR01 & 49 & duplicate & $5 / 16 / 2003$ & $5 / 19 / 2003$ & 0.415 & 0.012 & 0.000 & 0.000 & $\mathrm{~nm}$ \\
\hline A14-CR01-50 & CR01 & 50 & sample & $5 / 16 / 2003$ & $5 / 16 / 2003$ & 973.558 & 0.548 & 0.000 & 0.000 & 0.000 \\
\hline A14-CR01-50 & CR01 & 50 & duplicate & $5 / 16 / 2003$ & $5 / 19 / 2003$ & 1867.552 & 1.386 & 0.000 & 0.000 & $\mathrm{~nm}$ \\
\hline
\end{tabular}




\begin{tabular}{|c|c|c|c|c|c|c|c|c|c|c|}
\hline Sample ID & Boring & Depth (ft) & Type & $\begin{array}{c}\text { Date } \\
\text { Collected }\end{array}$ & $\begin{array}{c}\text { Date } \\
\text { Analyzed }\end{array}$ & $\begin{array}{c}\text { PCE } \\
(\mathrm{mg} / \mathrm{kg})\end{array}$ & $\begin{array}{c}\text { TCE } \\
(\mathrm{mg} / \mathrm{kg})\end{array}$ & $\begin{array}{l}\text { C-DCE } \\
(\mathrm{mg} / \mathrm{kg})\end{array}$ & $\begin{array}{c}\text { TCA } \\
(\mathrm{mg} / \mathrm{kg})\end{array}$ & $\begin{array}{r}\text { 11DCE } \\
(\mathrm{mg} / \mathrm{kg})\end{array}$ \\
\hline A14-CR01-51 & CR01 & 51 & sample & $5 / 16 / 2003$ & $5 / 16 / 2003$ & 4234.570 & 1.092 & 0.000 & 0.000 & 0.000 \\
\hline A14-CR01-51 & CR01 & 51 & duplicate & $5 / 16 / 2003$ & $5 / 19 / 2003$ & 2565.825 & 0.712 & 0.000 & 0.000 & $\mathrm{~nm}$ \\
\hline A14-CR01-52 & CR01 & 52 & sample & $5 / 16 / 2003$ & $5 / 16 / 2003$ & 2.703 & 0.154 & 0.000 & 0.000 & 0.000 \\
\hline A14-CR01-52 & CR01 & 52 & duplicate & $5 / 16 / 2003$ & $5 / 19 / 2003$ & 8.553 & 0.129 & 0.000 & 0.000 & $\mathrm{~nm}$ \\
\hline A14-CR01-53 & CR01 & 53 & sample & $5 / 16 / 2003$ & $5 / 16 / 2003$ & 1.402 & 0.009 & 0.000 & 0.000 & 0.000 \\
\hline A14-CR01-53 & CR01 & 53 & duplicate & $5 / 16 / 2003$ & $5 / 19 / 2003$ & 1.330 & 0.027 & 0.000 & 0.000 & $\mathrm{~nm}$ \\
\hline A14-CR01-54 & CR01 & 54 & sample & $5 / 16 / 2003$ & $5 / 16 / 2003$ & 0.127 & 0.000 & 0.000 & 0.000 & 0.000 \\
\hline A14-CR01-54 & CR01 & 54 & duplicate & $5 / 16 / 2003$ & $5 / 19 / 2003$ & 0.138 & 0.000 & 0.000 & 0.000 & $\mathrm{~nm}$ \\
\hline A14-CR01-55 & CR01 & 55 & sample & $5 / 16 / 2003$ & $5 / 16 / 2003$ & 0.219 & 0.003 & 0.000 & 0.000 & 0.000 \\
\hline A14-CR01-55 & CR01 & 55 & duplicate & $5 / 16 / 2003$ & $5 / 19 / 2003$ & 0.393 & 0.007 & 0.000 & 0.000 & $\mathrm{~nm}$ \\
\hline A14-CR01-56 & CR01 & 56 & sample & $5 / 16 / 2003$ & $5 / 16 / 2003$ & 0.142 & 0.000 & 0.000 & 0.000 & 0.000 \\
\hline A14-CR01-56 & CR01 & 56 & duplicate & $5 / 16 / 2003$ & $5 / 19 / 2003$ & 0.134 & 0.000 & 0.000 & 0.000 & $\mathrm{~nm}$ \\
\hline A14-CR01-57 & CR01 & 57 & sample & $5 / 16 / 2003$ & $5 / 16 / 2003$ & 0.708 & 0.007 & 0.000 & 0.000 & 0.000 \\
\hline A14- & CR01 & 57 & duplicate & $5 / 16 / 2003$ & $5 / 19 / 2003$ & 0.390 & 0.007 & 0.000 & 0.000 & $\mathrm{~nm}$ \\
\hline A14-CR01-58 & CR01 & 58 & sample & $5 / 16 / 2003$ & $5 / 16 / 2003$ & 0.256 & 0.005 & 0.000 & 0.000 & 0.000 \\
\hline A14-CR01-58 & CR01 & 58 & duplicate & $5 / 16 / 2003$ & $5 / 19 / 2003$ & 0.378 & 0.009 & 0.000 & 0.000 & $\mathrm{~nm}$ \\
\hline A14-CR01-59 & CR01 & 59 & sam & $5 / 16 / 2003$ & $5 / 16 / 2003$ & 0.522 & 0.007 & 0.000 & 0.000 & 0.000 \\
\hline A14-CR01-59 & CR01 & 59 & duplicate & $5 / 16 / 2003$ & $5 / 19 / 2003$ & 0.579 & 0.010 & 0.000 & 0.000 & $\mathrm{~nm}$ \\
\hline A14-CR01-60 & CR01 & 60 & sample & $5 / 16 / 2003$ & $5 / 16 / 2003$ & 0.028 & 0.000 & 0.000 & 0.000 & 0.000 \\
\hline A14-CR01-60 & CR01 & 60 & duplicate & $5 / 16 / 2003$ & $5 / 19 / 2003$ & 0.038 & 0.000 & 0.000 & 0.000 & $\mathrm{~nm}$ \\
\hline A14-CR01-61 & CR01 & 61 & sample & $5 / 16 / 2003$ & $5 / 16 / 2003$ & 0.193 & 0.002 & 0.000 & 0.000 & 0.000 \\
\hline A14-CR01-61 & R01 & 61 & duplicate & $5 / 16 / 2003$ & $5 / 19 / 2003$ & 7.163 & 0.064 & 0.000 & 0.000 & $\mathrm{~nm}$ \\
\hline A14-CR01 & CR01 & 62 & sample & $5 / 16 / 2003$ & $5 / 16 / 2003$ & 0.021 & 0.000 & 0.000 & 0.000 & 0.000 \\
\hline A14-CR01-62 & CR01 & 62 & duplicate & $5 / 16 / 2003$ & $5 / 19 / 2003$ & 0.053 & 0.000 & 0.000 & 0.000 & $\mathrm{~nm}$ \\
\hline A14-CR01-63 & CR01 & 63 & sample & $5 / 16 / 2003$ & $5 / 16 / 2003$ & 1.472 & 0.016 & 0.000 & 0.000 & 0.000 \\
\hline A14-CR01-63 & CR01 & 63 & duplicate & $5 / 16 / 2003$ & $5 / 19 / 2003$ & 7.961 & 0.110 & 0.000 & 0.000 & $\mathrm{~nm}$ \\
\hline A14-CR01-64 & CR01 & 64 & sam & $5 / 16 / 2003$ & $5 / 16 / 2003$ & 0.098 & 0.000 & 0.000 & 0.000 & 0.000 \\
\hline A14-CR01-64 & CR01 & 64 & duplicate & $5 / 16 / 2003$ & $5 / 19 / 2003$ & 0.110 & 0.000 & 0.000 & 0.000 & $\mathrm{~nm}$ \\
\hline A14-CR01-65 & CR01 & 65 & sample & $5 / 16 / 2003$ & $5 / 16 / 2003$ & 0.551 & 0.004 & 0.000 & 0.000 & 0.000 \\
\hline A14-CR01-65 & CR01 & 65 & duplicate & $5 / 16 / 2003$ & $5 / 19 / 2003$ & 0.666 & 0.009 & 0.000 & 0.000 & $\mathrm{~nm}$ \\
\hline A14-CR01-66 & CR01 & 66 & sample & $5 / 16 / 2003$ & $5 / 16 / 2003$ & 0.118 & 0.000 & 0.000 & 0.000 & 0.000 \\
\hline
\end{tabular}




\begin{tabular}{|c|c|c|c|c|c|c|c|c|c|c|}
\hline Sample ID & Boring & Depth (ft) & Type & $\begin{array}{c}\text { Date } \\
\text { Collected }\end{array}$ & $\begin{array}{c}\text { Date } \\
\text { Analyzed }\end{array}$ & $\begin{array}{c}\text { PCE } \\
(\mathrm{mg} / \mathrm{kg})\end{array}$ & $\begin{array}{c}\text { TCE } \\
(\mathrm{mg} / \mathrm{kg})\end{array}$ & $\begin{array}{l}\text { C-DCE } \\
(\mathrm{mg} / \mathrm{kg})\end{array}$ & $\begin{array}{c}\text { TCA } \\
(\mathrm{mg} / \mathrm{kg})\end{array}$ & $\begin{array}{l}\text { 11DCE } \\
(\mathrm{mg} / \mathrm{kg})\end{array}$ \\
\hline A14-CR01-66 & CR01 & 66 & duplicate & $5 / 16 / 2003$ & $5 / 19 / 2003$ & 0.051 & 0.000 & 0.000 & 0.000 & $\mathrm{~nm}$ \\
\hline A14-CR01-67 & CR01 & 67 & sample & $5 / 16 / 2003$ & $5 / 16 / 2003$ & 0.155 & 0.000 & 0.000 & 0.000 & 0.000 \\
\hline A14-CR01-67 & CR01 & 67 & duplicate & $5 / 16 / 2003$ & $5 / 19 / 2003$ & 0.128 & 0.000 & 0.000 & 0.000 & $\mathrm{~nm}$ \\
\hline A14-CR01-68 & CR01 & 68 & sample & $5 / 16 / 2003$ & $5 / 16 / 2003$ & 0.030 & 0.000 & 0.000 & 0.000 & 0.000 \\
\hline A14-CR01-68 & CR01 & 68 & duplicate & $5 / 16 / 2003$ & $5 / 19 / 2003$ & 0.070 & 0.000 & 0.000 & 0.000 & $\mathrm{~nm}$ \\
\hline$\overline{A 14-C}$ & CR01 & 69 & sample & $5 / 1$ & $5 / 16 / 2003$ & 0.194 & 0.000 & 0.000 & 0.000 & 0.000 \\
\hline A14-C & CR01 & 69 & duplicate & $5 / 16 / 2003$ & $5 / 19 / 2003$ & 0.364 & 0.004 & 0.000 & 0.000 & $\mathrm{~nm}$ \\
\hline A14-CR01-70 & CR01 & 70 & sample & $5 / 16 / 2003$ & $5 / 16 / 2003$ & 0.144 & 0.000 & 0.000 & 0.000 & 0.000 \\
\hline A14-CR01-70 & CR01 & 70 & duplicate & $5 / 16 / 2003$ & $5 / 19 / 2003$ & 0.197 & 0.000 & 0.000 & 0.000 & $\mathrm{~nm}$ \\
\hline A14-C & CR01 & 92 & sample & $5 / 16 / 2003$ & $5 / 16 / 2003$ & 0.903 & 0.005 & 0.000 & 0.000 & 0.000 \\
\hline A14-C & CR01 & 92 & duplicate & $5 / 16 / 2003$ & $5 / 19 / 2003$ & 1.362 & 0.012 & 0.000 & 0.000 & $\mathrm{~nm}$ \\
\hline A14-CR01-93 & CR01 & 93 & sample & $5 / 16 / 2003$ & $5 / 16 / 2003$ & 0.466 & 0.000 & 0.000 & 0.000 & 0.000 \\
\hline A14-CR01-93 & CR01 & 93 & duplicate & $5 / 16 / 2003$ & $5 / 19 / 2003$ & 0.430 & 0.000 & 0.000 & 0.000 & $\mathrm{~nm}$ \\
\hline A14-CR01-BLANK & CR01 & & blank & $5 / 15 / 2003$ & $5 / 15 / 2003$ & 0.000 & 0.000 & 0.000 & 0.000 & 0.000 \\
\hline A14-CR01-BLANK & CR01 & & blank & $5 / 15 / 2003$ & $5 / 19 / 2003$ & 0.000 & 0.000 & 0.000 & 0.000 & $\mathrm{~nm}$ \\
\hline
\end{tabular}


Table 7 - Sediment Analysis Results for Boring CR02

\begin{tabular}{|c|c|c|c|c|c|c|c|c|c|c|}
\hline Sample ID & Boring & Depth (ft) & Type & $\begin{array}{c}\text { Date } \\
\text { Collected }\end{array}$ & $\begin{array}{c}\text { Date } \\
\text { Analyzed }\end{array}$ & $\begin{array}{c}\text { PCE } \\
(\mathrm{mg} / \mathrm{kg})\end{array}$ & $\begin{array}{c}\text { TCE } \\
(\mathrm{mg} / \mathrm{kg})\end{array}$ & $\begin{array}{c}\text { C-DCE } \\
\text { (mg/kg) }\end{array}$ & $\begin{array}{c}\text { TCA } \\
(\mathrm{mg} / \mathrm{kg})\end{array}$ & $\begin{array}{l}11 \mathrm{DCE} \\
(\mathrm{mg} / \mathrm{kg})\end{array}$ \\
\hline A14-CR02-01 & CR02 & 1 & sample & $5 / 19 / 2003$ & $5 / 22 / 2003$ & 0.008 & 0.000 & 0.000 & 0.000 & 0.000 \\
\hline A14-CR02-01 & CR02 & 1 & duplicate & $5 / 19 / 2003$ & $6 / 4 / 2003$ & 0.006 & 0.000 & 0.000 & 0.000 & 0.000 \\
\hline A14-CR02-02 & CR02 & 2 & sample & $5 / 19 / 2003$ & $5 / 22 / 2003$ & 0.051 & 0.000 & 0.000 & 0.000 & 0.000 \\
\hline A14-CR02-02 & CR02 & 2 & duplicate & $5 / 19 / 2003$ & $6 / 4 / 2003$ & 0.077 & 0.002 & 0.000 & 0.000 & 0.000 \\
\hline A14-CR02-03 & CR02 & 3 & sample & $5 / 19 / 2003$ & $5 / 22 / 2003$ & 0.124 & 0.004 & 0.000 & 0.000 & 0.000 \\
\hline A14-CR02-03 & CR02 & 3 & duplicate & $5 / 19 / 2003$ & $6 / 4 / 2003$ & 0.258 & 0.009 & 0.000 & 0.000 & 0.000 \\
\hline A14-CR02-04 & CR02 & 4 & sample & $5 / 19 / 2003$ & $5 / 22 / 2003$ & 0.361 & 0.011 & 0.067 & 0.000 & 0.000 \\
\hline A14-CR02-04 & CR02 & 4 & duplicate & $5 / 19 / 2003$ & $6 / 4 / 2003$ & 0.509 & 0.025 & 0.107 & 0.000 & 0.000 \\
\hline A14-CR02-05 & CR02 & 5 & sample & $5 / 19 / 2003$ & $5 / 22 / 2003$ & 2.565 & 0.156 & 0.540 & 0.000 & 0.000 \\
\hline A14-CR02-05 & CR02 & 5 & duplicate & $5 / 19 / 2003$ & $6 / 4 / 2003$ & 1.855 & 0.102 & 0.724 & 0.000 & 0.000 \\
\hline A14-CR02-06 & CR02 & 6 & sample & $5 / 19 / 2003$ & $5 / 22 / 2003$ & 1.011 & 0.073 & 0.496 & 0.000 & 0.000 \\
\hline A14-CR02-06 & CR02 & 6 & duplicate & $5 / 19 / 2003$ & $6 / 4 / 2003$ & 0.994 & 0.100 & 1.083 & 0.000 & 0.000 \\
\hline A14-CR02-07 & CR02 & 7 & sample & $5 / 19 / 2003$ & $5 / 22 / 2003$ & 1.321 & 0.143 & 0.631 & 0.000 & 0.000 \\
\hline A14-CR02-07 & CR02 & 7 & duplicate & $5 / 19 / 2003$ & $6 / 4 / 2003$ & 1.430 & 0.147 & 1.401 & 0.000 & 0.000 \\
\hline A14-CR02-08 & CR02 & 8 & sample & $5 / 19 / 2003$ & $5 / 22 / 2003$ & 1.142 & 0.153 & 0.488 & 0.000 & 0.000 \\
\hline A14-CR02-08 & CR02 & 8 & duplicate & $5 / 19 / 2003$ & $6 / 4 / 2003$ & 0.662 & 0.103 & 0.493 & 0.000 & 0.000 \\
\hline A14-CR02-09 & CR02 & 9 & sample & $5 / 19 / 2003$ & $5 / 22 / 2003$ & 2.072 & 0.064 & 0.381 & 0.000 & 0.000 \\
\hline A14-CR02-09 & CR02 & 9 & duplicate & $5 / 19 / 2003$ & $6 / 4 / 2003$ & 2.290 & 0.113 & 0.869 & 0.000 & 0.000 \\
\hline A14-CR02-10 & CR02 & 10 & sample & $5 / 19 / 2003$ & $5 / 22 / 2003$ & 39.680 & 0.059 & 0.310 & 0.000 & 0.000 \\
\hline A14-CR02-10 & CR02 & 10 & duplicate & $5 / 19 / 2003$ & $6 / 4 / 2003$ & 36.755 & 0.073 & 0.429 & 0.000 & 0.000 \\
\hline A14-CR02-11 & CR02 & 11 & sample & $5 / 19 / 2003$ & $5 / 22 / 2003$ & 53.553 & 0.017 & 0.267 & 0.000 & 0.000 \\
\hline A14-CR02-11 & CR02 & 11 & duplicate & $5 / 19 / 2003$ & $6 / 4 / 2003$ & 47.820 & 0.024 & 0.267 & 0.000 & 0.000 \\
\hline A14-CR02-12 & CR02 & 12 & sample & $5 / 19 / 2003$ & $5 / 22 / 2003$ & 20.116 & 0.022 & 0.105 & 0.000 & 0.000 \\
\hline A14-CR02-12 & CR02 & 12 & duplicate & $5 / 19 / 2003$ & $6 / 4 / 2003$ & 19.874 & 0.027 & 0.000 & 0.000 & 0.000 \\
\hline A14-CR02-13 & CR02 & 13 & sample & $5 / 19 / 2003$ & $5 / 22 / 2003$ & 9.794 & 0.017 & 0.000 & 0.000 & 0.000 \\
\hline A14-CR02-13 & CR02 & 13 & duplicate & $5 / 19 / 2003$ & $6 / 4 / 2003$ & 21.666 & 0.042 & 0.000 & 0.000 & 0.000 \\
\hline A14-CR02-14 & CR02 & 14 & sample & $5 / 19 / 2003$ & $5 / 22 / 2003$ & 50.769 & 0.030 & 0.082 & 0.000 & 0.000 \\
\hline A14-CR02-14 & CR02 & 14 & duplicate & $5 / 19 / 2003$ & $6 / 4 / 2003$ & 18.164 & 0.026 & 0.000 & 0.000 & 0.000 \\
\hline
\end{tabular}




\begin{tabular}{|c|c|c|c|c|c|c|c|c|c|c|}
\hline Sample ID & Boring & Depth (ft) & Type & $\begin{array}{c}\text { Date } \\
\text { Collected }\end{array}$ & $\begin{array}{c}\text { Date } \\
\text { Analyzed }\end{array}$ & $\begin{array}{c}\text { PCE } \\
(\mathrm{mg} / \mathrm{kg})\end{array}$ & $\begin{array}{c}\text { TCE } \\
(\mathrm{mg} / \mathrm{kg})\end{array}$ & $\begin{array}{l}\text { C-DCE } \\
(\mathrm{mg} / \mathrm{kg}) \\
\end{array}$ & $\begin{array}{c}\text { TCA } \\
(\mathrm{mg} / \mathrm{kg})\end{array}$ & $\begin{array}{r}\text { 11DCE } \\
(\mathrm{mg} / \mathrm{kg})\end{array}$ \\
\hline A14-CR02-15 & CR02 & 15 & sample & $5 / 19 / 2003$ & $5 / 22 / 2003$ & 13.572 & 0.018 & 0.640 & 0.000 & 0.000 \\
\hline A14-CR02-15 & CR02 & 15 & duplicate & $5 / 19 / 2003$ & $6 / 4 / 2003$ & 26.605 & 0.039 & 0.198 & 0.000 & 0.000 \\
\hline A14-CR02-16 & CR02 & 16 & sample & $5 / 19 / 2003$ & $5 / 22 / 2003$ & 34.887 & 0.040 & 0.173 & 0.000 & 0.000 \\
\hline A14-CR02-16 & CR02 & 16 & duplicate & $5 / 19 / 2003$ & $6 / 4 / 2003$ & 31.370 & 0.058 & 0.293 & 0.000 & 0.000 \\
\hline A14-CR02-17 & CR02 & 17 & sample & $5 / 19 / 2003$ & $5 / 22 / 2003$ & 2374.321 & 0.728 & 1.169 & 0.007 & 0.000 \\
\hline A14-CR02-17 & CR02 & 17 & duplicate & $5 / 19 / 2003$ & $6 / 4 / 2003$ & 2867.347 & 0.745 & 1.335 & 0.009 & 0.000 \\
\hline A14-CR02-18 & CR02 & 18 & sample & $5 / 19 / 2003$ & $5 / 22 / 2003$ & 1742.729 & 0.320 & 0.315 & 0.078 & 0.000 \\
\hline A14-CR02-18 & CR02 & 18 & duplicate & $5 / 19 / 2003$ & $6 / 4 / 2003$ & 1593.242 & 0.197 & 1.796 & 0.092 & 0.000 \\
\hline A14-CR02-19 & CR02 & 19 & sample & $5 / 19 / 2003$ & $5 / 22 / 2003$ & na & na & na & na & 0.000 \\
\hline A14-CR02-19 & CR02 & 19 & duplicate & $5 / 19 / 2003$ & $6 / 4 / 2003$ & 1571.750 & 0.865 & 0.209 & 0.425 & 0.000 \\
\hline A14-CR02-20 & CR02 & 20 & sample & $5 / 19 / 2003$ & $5 / 22 / 2003$ & na & na & na & na & 0.000 \\
\hline A14-CR02-20 & CR02 & 20 & duplicate & $5 / 19 / 2003$ & $6 / 4 / 2003$ & 1801.207 & 1.813 & 0.402 & 0.359 & 0.000 \\
\hline A14-CR02-21 & CR02 & 21 & sample & $5 / 19 / 2003$ & $5 / 22 / 2003$ & 3181.346 & 1.465 & 0.071 & 0.041 & 0.000 \\
\hline A14-CR02-21 & CR02 & 21 & duplicate & $5 / 19 / 2003$ & $6 / 4 / 2003$ & 2216.910 & 1.145 & 0.140 & 0.062 & 0.000 \\
\hline A14-CR02-22 & CR02 & 22 & sample & $5 / 19 / 2003$ & $5 / 22 / 2003$ & 50.365 & 0.019 & 0.000 & 0.000 & 0.000 \\
\hline A14-CR02-22 & CR02 & 22 & duplicate & $5 / 19 / 2003$ & $6 / 4 / 2003$ & 36.496 & 0.009 & 0.000 & 0.000 & 0.000 \\
\hline A14-CR02-23 & CR02 & 23 & sample & $5 / 19 / 2003$ & $5 / 22 / 2003$ & 2.188 & 0.000 & 0.000 & 0.000 & 0.000 \\
\hline A14-CR02-23 & CR02 & 23 & duplicate & $5 / 19 / 2003$ & $6 / 4 / 2003$ & 2.224 & 0.000 & 0.000 & 0.000 & 0.000 \\
\hline A14-CR02-24 & CR02 & 24 & sample & $5 / 19 / 2003$ & $5 / 22 / 2003$ & 14.866 & 0.007 & 0.000 & 0.000 & 0.000 \\
\hline A14-CR02-24 & CR02 & 24 & duplicate & $5 / 19 / 2003$ & $6 / 4 / 2003$ & 2.969 & 0.003 & 0.000 & 0.000 & 0.000 \\
\hline A14-CR02-25 & CR02 & 25 & sample & $5 / 19 / 2003$ & $5 / 22 / 2003$ & 6.708 & 0.000 & 0.000 & 0.000 & 0.000 \\
\hline A14-CR02-25 & CR02 & 25 & duplicate & $5 / 19 / 2003$ & $6 / 4 / 2003$ & 8.231 & 0.003 & 0.000 & 0.000 & 0.000 \\
\hline A14-CR02-27 & CR02 & 27 & sample & $5 / 19 / 2003$ & $5 / 22 / 2003$ & 0.058 & 0.005 & 0.000 & 0.000 & 0.000 \\
\hline A14-CR02-27 & CR02 & 27 & duplicate & $5 / 19 / 2003$ & $6 / 4 / 2003$ & 0.084 & 0.000 & 0.000 & 0.000 & 0.000 \\
\hline A14-CR02-28 & CR02 & 28 & sample & $5 / 19 / 2003$ & $5 / 22 / 2003$ & 0.034 & 0.000 & 0.000 & 0.000 & 0.000 \\
\hline A14-CR02-28 & CR02 & 28 & duplicate & $5 / 19 / 2003$ & $6 / 4 / 2003$ & 0.050 & 0.000 & 0.000 & 0.000 & 0.000 \\
\hline A14-CR02-29 & CR02 & 29 & sample & $5 / 19 / 2003$ & $5 / 22 / 2003$ & 0.031 & 0.000 & 0.000 & 0.000 & 0.000 \\
\hline A14-CR02-29 & CR02 & 29 & duplicate & $5 / 19 / 2003$ & $6 / 4 / 2003$ & 0.050 & 0.000 & 0.000 & 0.000 & 0.000 \\
\hline A14-CR02-30 & CR02 & 30 & sample & $5 / 19 / 2003$ & $5 / 22 / 2003$ & 0.033 & 0.000 & 0.000 & 0.000 & 0.000 \\
\hline A14-CR02-30 & CR02 & 30 & duplicate & $5 / 19 / 2003$ & $6 / 4 / 2003$ & 0.038 & 0.000 & 0.000 & 0.000 & 0.000 \\
\hline A14-CR02-31 & CR02 & 31 & sample & $5 / 19 / 2003$ & $5 / 22 / 2003$ & 0.021 & 0.000 & 0.000 & 0.000 & 0.000 \\
\hline
\end{tabular}




\begin{tabular}{|c|c|c|c|c|c|c|c|c|c|c|}
\hline Sample ID & Boring & Depth (ft) & Type & $\begin{array}{c}\text { Date } \\
\text { Collected }\end{array}$ & $\begin{array}{c}\text { Date } \\
\text { Analyzed }\end{array}$ & $\begin{array}{c}\text { PCE } \\
(\mathrm{mg} / \mathrm{kg})\end{array}$ & $\begin{array}{c}\text { TCE } \\
(\mathrm{mg} / \mathrm{kg})\end{array}$ & $\begin{array}{l}\text { C-DCE } \\
(\mathrm{mg} / \mathrm{kg}) \\
\end{array}$ & $\begin{array}{c}\text { TCA } \\
(\mathrm{mg} / \mathrm{kg})\end{array}$ & $\begin{array}{r}\text { 11DCE } \\
(\mathrm{mg} / \mathrm{kg})\end{array}$ \\
\hline A14-CR02-31 & CR02 & 31 & duplicate & $5 / 19 / 2003$ & 6/4/2003 & 0.027 & 0.000 & 0.000 & 0.000 & 0.000 \\
\hline A14-CR02-32 & CR02 & 32 & sample & $5 / 19 / 2003$ & $5 / 22 / 2003$ & 0.021 & 0.000 & 0.000 & 0.000 & 0.000 \\
\hline A14-CR02-32 & CR02 & 32 & duplicate & $5 / 19 / 2003$ & $6 / 4 / 2003$ & 0.026 & 0.000 & 0.000 & 0.000 & 0.000 \\
\hline A14-CR02-33 & CR02 & 33 & sample & $5 / 19 / 2003$ & $5 / 22 / 2003$ & 0.017 & 0.000 & 0.000 & 0.000 & 0.000 \\
\hline A14-CR02-33 & CR02 & 33 & duplicate & $5 / 19 / 2003$ & $6 / 4 / 2003$ & 0.024 & 0.000 & 0.000 & 0.000 & 0.000 \\
\hline A14-CR02-34 & CR02 & 34 & sample & $5 / 19 / 2003$ & $5 / 22 / 2003$ & 0.020 & 0.000 & 0.000 & 0.000 & 0.000 \\
\hline A14-CR02-34 & CR02 & 34 & duplicate & $5 / 19 / 2003$ & $6 / 4 / 2003$ & 0.025 & 0.000 & 0.000 & 0.000 & 0.000 \\
\hline A14-CR02-35 & CR02 & 35 & sample & $5 / 19 / 2003$ & $5 / 22 / 2003$ & 0.013 & 0.000 & 0.000 & 0.000 & 0.000 \\
\hline A14-CR02-35 & CR02 & 35 & duplicate & $5 / 19 / 2003$ & $6 / 4 / 2003$ & 0.019 & 0.000 & 0.000 & 0.000 & 0.000 \\
\hline A14-CR02-36 & CR02 & 36 & sample & $5 / 19 / 2003$ & $5 / 22 / 2003$ & 0.011 & 0.000 & 0.000 & 0.000 & 0.000 \\
\hline A14-CR02-36 & CR02 & 36 & duplicate & $5 / 19 / 2003$ & $6 / 4 / 2003$ & 0.018 & 0.000 & 0.000 & 0.000 & 0.000 \\
\hline A14-CR02-37 & CR02 & 37 & sample & $5 / 19 / 2003$ & $5 / 22 / 2003$ & 0.013 & 0.000 & 0.000 & 0.000 & 0.000 \\
\hline A14-CR02-37 & CR02 & 37 & duplicate & $5 / 19 / 2003$ & $6 / 4 / 2003$ & 0.015 & 0.000 & 0.000 & 0.000 & 0.000 \\
\hline A14-CR02-38 & CR02 & 38 & sample & $5 / 19 / 2003$ & $5 / 22 / 2003$ & 0.019 & 0.000 & 0.000 & 0.000 & 0.000 \\
\hline A14-CR02-38 & CR02 & 38 & duplicate & $5 / 19 / 2003$ & $6 / 4 / 2003$ & 0.016 & 0.000 & 0.000 & 0.000 & 0.000 \\
\hline A14-CR02-39 & CR02 & 39 & sample & $5 / 19 / 2003$ & $5 / 22 / 2003$ & 0.012 & 0.000 & 0.000 & 0.000 & 0.000 \\
\hline A14-CR02-39 & CR02 & 39 & duplicate & $5 / 19 / 2003$ & $6 / 4 / 2003$ & 0.013 & 0.000 & 0.000 & 0.000 & 0.000 \\
\hline A14-CR02-40 & CR02 & 40 & sample & $5 / 19 / 2003$ & $5 / 22 / 2003$ & 0.009 & 0.000 & 0.000 & 0.000 & 0.000 \\
\hline A14-CR02-40 & CR02 & 40 & duplicate & $5 / 19 / 2003$ & $6 / 4 / 2003$ & 0.011 & 0.000 & 0.000 & 0.000 & 0.000 \\
\hline A14-CR02-41 & CR02 & 41 & sample & $5 / 19 / 2003$ & $5 / 22 / 2003$ & 0.012 & 0.000 & 0.000 & 0.000 & 0.000 \\
\hline A14-CR02-41 & CR02 & 41 & duplicate & $5 / 19 / 2003$ & $6 / 4 / 2003$ & 0.013 & 0.000 & 0.000 & 0.000 & 0.000 \\
\hline A14-CR02-42 & CR02 & 42 & sample & $5 / 19 / 2003$ & $5 / 22 / 2003$ & 0.010 & 0.000 & 0.000 & 0.000 & 0.000 \\
\hline A14-CR02-42 & CR02 & 42 & duplicate & $5 / 19 / 2003$ & $6 / 4 / 2003$ & 0.016 & 0.000 & 0.000 & 0.000 & 0.000 \\
\hline A14-CR02-43 & CR02 & 43 & sample & $5 / 19 / 2003$ & $5 / 22 / 2003$ & 0.012 & 0.000 & 0.000 & 0.000 & 0.000 \\
\hline A14-CR02-43 & CR02 & 43 & duplicate & $5 / 19 / 2003$ & $6 / 4 / 2003$ & 0.013 & 0.000 & 0.000 & 0.000 & 0.000 \\
\hline A14-CR02-44 & CR02 & 44 & sample & $5 / 19 / 2003$ & $5 / 22 / 2003$ & 0.011 & 0.000 & 0.000 & 0.000 & 0.000 \\
\hline A14-CR02-44 & CR02 & 44 & duplicate & $5 / 19 / 2003$ & $6 / 4 / 2003$ & 0.012 & 0.000 & 0.000 & 0.000 & 0.000 \\
\hline A14-CR02-45 & CR02 & 45 & sample & $5 / 19 / 2003$ & $5 / 22 / 2003$ & 0.011 & 0.000 & 0.000 & 0.000 & 0.000 \\
\hline A14-CR02-45 & CR02 & 45 & duplicate & $5 / 19 / 2003$ & $6 / 4 / 2003$ & 0.010 & 0.000 & 0.000 & 0.000 & 0.000 \\
\hline A14-CR02-46 & CR02 & 46 & sample & $5 / 19 / 2003$ & $5 / 22 / 2003$ & 0.004 & 0.000 & 0.000 & 0.000 & 0.000 \\
\hline A14-CR02-46 & CR02 & 46 & duplicate & $5 / 19 / 2003$ & $6 / 4 / 2003$ & 0.010 & 0.000 & 0.000 & 0.000 & 0.000 \\
\hline
\end{tabular}




\begin{tabular}{|c|c|c|c|c|c|c|c|c|c|c|}
\hline Sample ID & Boring & Depth (ft) & Type & $\begin{array}{c}\text { Date } \\
\text { Collected }\end{array}$ & $\begin{array}{c}\text { Date } \\
\text { Analyzed }\end{array}$ & $\begin{array}{c}\text { PCE } \\
(\mathrm{mg} / \mathrm{kg})\end{array}$ & $\begin{array}{c}\text { TCE } \\
(\mathrm{mg} / \mathrm{kg})\end{array}$ & $\begin{array}{l}\text { C-DCE } \\
(\mathrm{mg} / \mathrm{kg})\end{array}$ & $\begin{array}{c}\text { TCA } \\
(\mathrm{mg} / \mathrm{kg})\end{array}$ & $\begin{array}{l}\text { 11DCE } \\
(\mathrm{mg} / \mathrm{kg})\end{array}$ \\
\hline A14-CR02-47 & CR02 & 47 & sample & $5 / 19 / 2003$ & $5 / 22 / 2003$ & 0.012 & 0.000 & 0.000 & 0.000 & 0.000 \\
\hline A14-CR02-47 & CR02 & 47 & duplicate & $5 / 19 / 2003$ & $6 / 4 / 2003$ & 0.009 & 0.000 & 0.000 & 0.000 & 0.000 \\
\hline A14-CR02-48 & CR02 & 48 & sample & $5 / 19 / 2003$ & $5 / 22 / 2003$ & 0.010 & 0.000 & 0.000 & 0.000 & 0.000 \\
\hline A14-CR02-48 & CR02 & 48 & duplicate & $5 / 19 / 2003$ & $6 / 4 / 2003$ & 0.009 & 0.000 & 0.000 & 0.000 & 0.000 \\
\hline A14-CR02-49 & CR02 & 49 & sample & $5 / 19 / 2003$ & $5 / 22 / 2003$ & 0.009 & 0.000 & 0.000 & 0.000 & 0.000 \\
\hline A14-CR02-49 & CR02 & 49 & duplicate & $5 / 19 / 2003$ & $6 / 4 / 2003$ & 0.009 & 0.000 & 0.000 & 0.000 & 0.000 \\
\hline A14-CR02-50 & CR02 & 50 & sample & $5 / 19 / 2003$ & $5 / 22 / 2003$ & 0.007 & 0.000 & 0.000 & 0.000 & 0.000 \\
\hline A14-CR02-50 & CR02 & 50 & duplicate & $5 / 19 / 2003$ & $6 / 4 / 2003$ & 0.008 & 0.000 & 0.000 & 0.000 & 0.000 \\
\hline A14-CR02-51 & CR02 & 51 & sample & $5 / 19 / 2003$ & $5 / 22 / 2003$ & 0.010 & 0.000 & 0.000 & 0.000 & 0.000 \\
\hline A14-CR02-51 & CR02 & 51 & duplicate & $5 / 19 / 2003$ & $6 / 4 / 2003$ & 0.007 & 0.000 & 0.000 & 0.000 & 0.000 \\
\hline A14-CR02-52 & CR02 & 52 & sample & $5 / 19 / 2003$ & $5 / 22 / 2003$ & 0.010 & 0.000 & 0.000 & 0.000 & 0.000 \\
\hline A14-CR02-52 & CR02 & 52 & duplicate & $5 / 19 / 2003$ & $6 / 4 / 2003$ & 0.009 & 0.000 & 0.000 & 0.000 & 0.000 \\
\hline A14-CR02-53 & CR02 & 53 & sample & $5 / 19 / 2003$ & $5 / 22 / 2003$ & 0.011 & 0.000 & 0.000 & 0.000 & 0.000 \\
\hline A14-CR02-53 & CR02 & 53 & duplicate & $5 / 19 / 2003$ & $6 / 4 / 2003$ & 0.009 & 0.000 & 0.000 & 0.000 & 0.000 \\
\hline A14-CR02-54 & CR02 & 54 & sample & $5 / 19 / 2003$ & $5 / 22 / 2003$ & 0.042 & 0.000 & 0.000 & 0.000 & 0.000 \\
\hline A14-CR02-54 & CR02 & 54 & duplicate & $5 / 19 / 2003$ & $6 / 4 / 2003$ & 0.043 & 0.000 & 0.000 & 0.000 & 0.000 \\
\hline A14-CR02-55 & CR02 & 55 & sample & $5 / 19 / 2003$ & $5 / 22 / 2003$ & 0.434 & 0.000 & 0.000 & 0.000 & 0.000 \\
\hline A14-CR02-55 & CR02 & 55 & \begin{tabular}{|l} 
duplicate \\
\end{tabular} & $5 / 19 / 2003$ & $6 / 4 / 2003$ & 0.465 & 0.000 & 0.000 & 0.000 & 0.000 \\
\hline A14-CR02-56 & CR02 & 56 & sample & $5 / 19 / 2003$ & $5 / 22 / 2003$ & 0.504 & 0.000 & 0.000 & 0.000 & 0.000 \\
\hline A14-CR02-56 & CR02 & 56 & duplicate & $5 / 19 / 2003$ & $6 / 4 / 2003$ & 0.809 & 0.000 & 0.000 & 0.000 & 0.000 \\
\hline A14-CR02-57 & CR02 & 57 & sample & $5 / 19 / 2003$ & $5 / 22 / 2003$ & 1.602 & 0.000 & 0.000 & 0.000 & 0.000 \\
\hline A14-CR02-57 & CR02 & 57 & duplicate & $5 / 19 / 2003$ & $6 / 4 / 2003$ & 0.797 & 0.000 & 0.000 & 0.000 & 0.000 \\
\hline A14-CR02-58 & CR02 & 58 & sample & $5 / 19 / 2003$ & $5 / 22 / 2003$ & 3.800 & 0.000 & 0.000 & 0.000 & 0.000 \\
\hline A14-CR02-58 & CR02 & 58 & duplicate & $5 / 19 / 2003$ & $6 / 4 / 2003$ & 4.935 & 0.000 & 0.000 & 0.000 & 0.000 \\
\hline A14-CR02-59 & CR02 & 59 & sample & $5 / 19 / 2003$ & $5 / 22 / 2003$ & 2.712 & 0.000 & 0.000 & 0.000 & 0.000 \\
\hline A14-CR02-59 & CR02 & 59 & duplicate & $5 / 19 / 2003$ & $6 / 4 / 2003$ & 1.188 & 0.000 & 0.000 & 0.000 & 0.000 \\
\hline A14-CR02-60 & CR02 & 60 & sample & $5 / 19 / 2003$ & $5 / 22 / 2003$ & 0.938 & 0.000 & 0.000 & 0.000 & 0.000 \\
\hline A14-CR02-60 & CR02 & 60 & duplicate & $5 / 19 / 2003$ & $6 / 4 / 2003$ & 1.091 & 0.000 & 0.000 & 0.000 & 0.000 \\
\hline A14-CR02-61 & CR02 & 61 & sample & $5 / 19 / 2003$ & $5 / 22 / 2003$ & 0.360 & 0.000 & 0.000 & 0.000 & 0.000 \\
\hline A14-CR02-61 & CR02 & 61 & duplicate & $5 / 19 / 2003$ & $6 / 4 / 2003$ & 0.901 & 0.000 & 0.000 & 0.000 & 0.000 \\
\hline A14-CR02-062 & CR02 & 62 & sample & $5 / 19 / 2003$ & $5 / 22 / 2003$ & 0.019 & 0.000 & 0.000 & 0.000 & 0.000 \\
\hline
\end{tabular}




\begin{tabular}{|c|c|c|c|c|c|c|c|c|c|c|}
\hline Sample ID & Boring & Depth (ft) & Type & $\begin{array}{c}\text { Date } \\
\text { Collected }\end{array}$ & $\begin{array}{c}\text { Date } \\
\text { Analyzed }\end{array}$ & $\begin{array}{c}\text { PCE } \\
(\mathrm{mg} / \mathrm{kg})\end{array}$ & $\begin{array}{c}\text { TCE } \\
(\mathrm{mg} / \mathrm{kg})\end{array}$ & $\begin{array}{l}\text { C-DCE } \\
(\mathrm{mg} / \mathrm{kg}) \\
\end{array}$ & $\begin{array}{c}\text { TCA } \\
(\mathrm{mg} / \mathrm{kg})\end{array}$ & $\begin{array}{r}\text { 11DCE } \\
(\mathrm{mg} / \mathrm{kg})\end{array}$ \\
\hline A14-CR02-062 & CR02 & 62 & duplicate & $5 / 19 / 2003$ & $6 / 2 / 2003$ & 0.020 & 0.000 & 0.000 & 0.000 & 0.000 \\
\hline A14-CR02-063 & CR02 & 63 & sample & $5 / 19 / 2003$ & $5 / 22 / 2003$ & 0.040 & 0.000 & 0.000 & 0.000 & 0.000 \\
\hline A14-CR02-063 & CR02 & 63 & duplicate & $5 / 19 / 2003$ & $6 / 2 / 2003$ & 0.033 & 0.000 & 0.000 & 0.000 & 0.000 \\
\hline A14-CR02-064 & CR02 & 64 & sample & $5 / 19 / 2003$ & $5 / 22 / 2003$ & 0.015 & 0.000 & 0.000 & 0.000 & 0.000 \\
\hline A14-CR02-064 & CR02 & 64 & duplicate & $5 / 19 / 2003$ & $6 / 2 / 2003$ & 0.013 & 0.000 & 0.000 & 0.000 & 0.000 \\
\hline A14-CR02-065 & CR02 & 65 & sample & $5 / 19 / 2003$ & $5 / 22 / 2003$ & 0.007 & 0.000 & 0.000 & 0.000 & 0.000 \\
\hline A14-CR02-065 & CR02 & 65 & duplicate & $5 / 19 / 2003$ & $6 / 2 / 2003$ & 0.007 & 0.000 & 0.000 & 0.000 & 0.000 \\
\hline A14-CR02-066 & CR02 & 66 & sample & $5 / 19 / 2003$ & $5 / 22 / 2003$ & 0.005 & 0.000 & 0.000 & 0.000 & 0.000 \\
\hline A14-CR02-066 & CR02 & 66 & duplicate & $5 / 19 / 2003$ & $6 / 2 / 2003$ & 0.004 & 0.000 & 0.000 & 0.000 & 0.000 \\
\hline A14-CR02-067 & CR02 & 67 & sample & $5 / 19 / 2003$ & $5 / 22 / 2003$ & 0.008 & 0.000 & 0.000 & 0.000 & 0.000 \\
\hline A14-CR02-067 & CR02 & 67 & duplicate & $5 / 19 / 2003$ & $6 / 2 / 2003$ & 0.006 & 0.000 & 0.000 & 0.000 & 0.000 \\
\hline A14-CR02-068 & CR02 & 68 & sample & $5 / 19 / 2003$ & $5 / 22 / 2003$ & 0.009 & 0.000 & 0.000 & 0.000 & 0.000 \\
\hline A14-CR02-068 & CR02 & 68 & duplicate & $5 / 19 / 2003$ & $6 / 2 / 2003$ & 0.005 & 0.000 & 0.000 & 0.000 & 0.000 \\
\hline A14-CR02-069 & CR02 & 69 & sample & $5 / 19 / 2003$ & $5 / 22 / 2003$ & 0.000 & 0.000 & 0.000 & 0.000 & 0.000 \\
\hline A14-CR02-069 & CR02 & 69 & duplicate & $5 / 19 / 2003$ & $6 / 2 / 2003$ & 0.005 & 0.000 & 0.000 & 0.000 & 0.000 \\
\hline A14-CR02-070 & CR02 & 70 & sample & $5 / 19 / 2003$ & $5 / 22 / 2003$ & 0.000 & 0.000 & 0.000 & 0.000 & 0.000 \\
\hline A14-CR02-070 & CR02 & 70 & duplicate & $5 / 19 / 2003$ & $6 / 2 / 2003$ & 0.005 & 0.000 & 0.000 & 0.000 & 0.000 \\
\hline A14-CR02-071 & CR02 & 71 & sample & $5 / 19 / 2003$ & $5 / 22 / 2003$ & 0.009 & 0.000 & 0.000 & 0.000 & 0.000 \\
\hline A14-CR02-071 & CR02 & 71 & duplicate & $5 / 19 / 2003$ & $6 / 2 / 2003$ & 0.005 & 0.000 & 0.000 & 0.000 & 0.000 \\
\hline A14-CR02-072 & CR02 & 72 & sample & $5 / 19 / 2003$ & $5 / 22 / 2003$ & 0.007 & 0.000 & 0.000 & 0.000 & 0.000 \\
\hline A14-CR02-072 & CR02 & 72 & duplicate & $5 / 19 / 2003$ & $6 / 2 / 2003$ & 0.005 & 0.000 & 0.000 & 0.000 & 0.000 \\
\hline A14-CR02-073 & CR02 & 73 & sample & $5 / 19 / 2003$ & $5 / 22 / 2003$ & 0.006 & 0.000 & 0.000 & 0.000 & 0.000 \\
\hline A14-CR02-073 & CR02 & 73 & duplicate & $5 / 19 / 2003$ & $6 / 2 / 2003$ & 0.005 & 0.000 & 0.000 & 0.000 & 0.000 \\
\hline A14-CR02-074 & CR02 & 74 & sample & $5 / 19 / 2003$ & $5 / 22 / 2003$ & 0.005 & 0.000 & 0.000 & 0.000 & 0.000 \\
\hline A14-CR02-074 & CR02 & 74 & duplicate & $5 / 19 / 2003$ & $6 / 2 / 2003$ & 0.003 & 0.000 & 0.000 & 0.000 & 0.000 \\
\hline A14-CR02-075 & CR02 & 75 & sample & $5 / 19 / 2003$ & $5 / 22 / 2003$ & 0.009 & 0.000 & 0.000 & 0.000 & 0.000 \\
\hline A14-CR02-075 & CR02 & 75 & duplicate & $5 / 19 / 2003$ & $6 / 2 / 2003$ & 0.000 & 0.000 & 0.000 & 0.000 & 0.000 \\
\hline A14-CR02-076 & CR02 & 76 & sample & $5 / 19 / 2003$ & $5 / 22 / 2003$ & 0.006 & 0.000 & 0.000 & 0.000 & 0.000 \\
\hline A14-CR02-076 & CR02 & 76 & duplicate & $5 / 19 / 2003$ & $6 / 2 / 2003$ & 0.000 & 0.000 & 0.000 & 0.000 & 0.000 \\
\hline A14-CR02-077 & CR02 & 77 & sample & $5 / 19 / 2003$ & $5 / 22 / 2003$ & 0.007 & 0.000 & 0.000 & 0.000 & 0.000 \\
\hline A14-CR02-077 & CR02 & 77 & duplicate & $5 / 19 / 2003$ & $6 / 2 / 2003$ & 0.000 & 0.000 & 0.000 & 0.000 & 0.000 \\
\hline
\end{tabular}




\begin{tabular}{|c|c|c|c|c|c|c|c|c|c|c|}
\hline Sample ID & Boring & Depth (ft) & Type & $\begin{array}{c}\text { Date } \\
\text { Collected }\end{array}$ & $\begin{array}{c}\text { Date } \\
\text { Analyzed }\end{array}$ & $\begin{array}{c}\text { PCE } \\
(\mathrm{mg} / \mathrm{kg})\end{array}$ & $\begin{array}{c}\text { TCE } \\
(\mathrm{mg} / \mathrm{kg})\end{array}$ & $\begin{array}{l}\text { C-DCE } \\
(\mathrm{mg} / \mathrm{kg}) \\
\end{array}$ & $\begin{array}{c}\text { TCA } \\
(\mathrm{mg} / \mathrm{kg})\end{array}$ & $\begin{array}{r}\text { 11DCE } \\
(\mathrm{mg} / \mathrm{kg})\end{array}$ \\
\hline A14-CR02-078 & CR02 & 78 & sample & $5 / 19 / 2003$ & $5 / 22 / 2003$ & 0.007 & 0.000 & 0.000 & 0.000 & 0.000 \\
\hline A14-CR02-078 & CR02 & 78 & duplicate & $5 / 19 / 2003$ & $6 / 2 / 2003$ & 0.004 & 0.000 & 0.000 & 0.000 & 0.000 \\
\hline A14-CR02-079 & CR02 & 79 & sample & $5 / 19 / 2003$ & $5 / 22 / 2003$ & 0.004 & 0.000 & 0.000 & 0.000 & 0.000 \\
\hline A14-CR02-079 & CR02 & 79 & duplicate & $5 / 19 / 2003$ & $6 / 2 / 2003$ & 0.003 & 0.000 & 0.000 & 0.000 & 0.000 \\
\hline A14-CR02-080 & CR02 & 80 & sample & $5 / 19 / 2003$ & $5 / 22 / 2003$ & 0.003 & 0.000 & 0.000 & 0.000 & 0.000 \\
\hline A14-CR02-080 & CR02 & 80 & duplicate & $5 / 19 / 2003$ & $6 / 2 / 2003$ & 0.000 & 0.000 & 0.000 & 0.000 & 0.000 \\
\hline A14-CR02-095 & CR02 & 95 & sample & $5 / 19 / 2003$ & $5 / 22 / 2003$ & 0.008 & 0.000 & 0.000 & 0.000 & 0.000 \\
\hline A14-CR02-095 & CR02 & 95 & duplicate & $5 / 19 / 2003$ & $6 / 2 / 2003$ & 0.005 & 0.000 & 0.000 & 0.000 & 0.000 \\
\hline A14-CR02-096 & CR02 & 96 & sample & $5 / 19 / 2003$ & $5 / 22 / 2003$ & 0.000 & 0.000 & 0.000 & 0.000 & 0.000 \\
\hline A14-CR02-096 & CR02 & 96 & duplicate & $5 / 19 / 2003$ & $6 / 2 / 2003$ & 0.000 & 0.000 & 0.000 & 0.000 & 0.000 \\
\hline A14-CR02-098 & CR02 & 98 & sample & $5 / 19 / 2003$ & $5 / 22 / 2003$ & 1.535 & 0.009 & 0.000 & 0.000 & 0.000 \\
\hline A14-CR02-098 & CR02 & 98 & duplicate & $5 / 19 / 2003$ & $6 / 2 / 2003$ & 2.773 & 0.014 & 0.000 & 0.000 & 0.000 \\
\hline A14-CR02-099 & CR02 & 99 & sample & $5 / 19 / 2003$ & $5 / 22 / 2003$ & 0.008 & 0.000 & 0.000 & 0.000 & 0.000 \\
\hline A14-CR02-099 & CR02 & 99 & duplicate & $5 / 19 / 2003$ & $6 / 2 / 2003$ & 0.007 & 0.000 & 0.000 & 0.000 & 0.000 \\
\hline A14-CR02-100 & CR02 & 100 & sample & $5 / 19 / 2003$ & $5 / 22 / 2003$ & 0.003 & 0.000 & 0.000 & 0.000 & 0.000 \\
\hline A14-CR02-100 & CR02 & 100 & duplicate & $5 / 19 / 2003$ & $6 / 2 / 2003$ & 0.008 & 0.000 & 0.000 & 0.000 & 0.000 \\
\hline A14-CR02-102 & CR02 & 102 & sample & $5 / 19 / 2003$ & $5 / 22 / 2003$ & 0.019 & 0.000 & 0.000 & 0.000 & 0.000 \\
\hline A14-CR02-102 & CR02 & 102 & duplicate & $5 / 19 / 2003$ & $6 / 2 / 2003$ & 0.043 & 0.000 & 0.000 & 0.000 & 0.000 \\
\hline A14-CR02-103 & CR02 & 103 & sample & $5 / 19 / 2003$ & $5 / 22 / 2003$ & 2.005 & 0.007 & 0.000 & 0.000 & 0.000 \\
\hline A14-CR02-103 & CR02 & 103 & duplicate & $5 / 19 / 2003$ & $6 / 2 / 2003$ & 1.867 & 0.009 & 0.000 & 0.000 & 0.000 \\
\hline A14-CR02-105 & CR02 & 105 & sample & $5 / 19 / 2003$ & $5 / 22 / 2003$ & 11.376 & 0.181 & 0.000 & 0.000 & 0.000 \\
\hline A14-CR02-105 & CR02 & 105 & duplicate & $5 / 19 / 2003$ & $6 / 2 / 2003$ & 12.622 & 0.196 & 0.000 & 0.000 & 0.000 \\
\hline A14-CR02-106 & CR02 & 106 & sample & $5 / 19 / 2003$ & $5 / 22 / 2003$ & 0.036 & 0.000 & 0.000 & 0.000 & 0.000 \\
\hline A14-CR02-106 & CR02 & 106 & duplicate & $5 / 19 / 2003$ & $6 / 2 / 2003$ & 0.037 & 0.000 & 0.000 & 0.000 & 0.000 \\
\hline A14-CR02-107 & CR02 & 107 & sample & $5 / 19 / 2003$ & $5 / 22 / 2003$ & 0.033 & 0.004 & 0.000 & 0.000 & 0.000 \\
\hline A14-CR02-107 & CR02 & 107 & duplicate & $5 / 19 / 2003$ & $6 / 2 / 2003$ & 0.033 & 0.003 & 0.000 & 0.000 & 0.000 \\
\hline A14-CR02-108 & CR02 & 108 & sample & $5 / 19 / 2003$ & $5 / 22 / 2003$ & 0.008 & 0.000 & 0.000 & 0.000 & 0.000 \\
\hline A14-CR02-108 & CR02 & 108 & duplicate & $5 / 19 / 2003$ & $6 / 2 / 2003$ & 0.008 & 0.000 & 0.000 & 0.000 & 0.000 \\
\hline A14-CR02-108.5 & CR02 & 108.5 & sample & $5 / 19 / 2003$ & $5 / 22 / 2003$ & 0.039 & 0.006 & 0.000 & 0.000 & 0.000 \\
\hline A14-CR02-108.5 & CR02 & 108.5 & duplicate & $5 / 19 / 2003$ & $6 / 2 / 2003$ & 0.040 & 0.005 & 0.000 & 0.000 & 0.000 \\
\hline A14-CR02-109 & CR02 & 109 & sample & $5 / 19 / 2003$ & $5 / 22 / 2003$ & 0.003 & 0.000 & 0.000 & 0.000 & 0.000 \\
\hline
\end{tabular}




\begin{tabular}{|c|c|c|c|c|c|c|c|c|c|c|}
\hline Sample ID & Boring & Depth (ft) & Type & $\begin{array}{c}\text { Date } \\
\text { Collected }\end{array}$ & $\begin{array}{c}\text { Date } \\
\text { Analyzed }\end{array}$ & $\begin{array}{c}\text { PCE } \\
(\mathrm{mg} / \mathrm{kg})\end{array}$ & $\begin{array}{c}\text { TCE } \\
(\mathrm{mg} / \mathrm{kg})\end{array}$ & $\begin{array}{l}\text { C-DCE } \\
(\mathrm{mg} / \mathrm{kg})\end{array}$ & $\begin{array}{c}\text { TCA } \\
(\mathrm{mg} / \mathrm{kg})\end{array}$ & $\begin{array}{r}\text { 11DCE } \\
\text { (mg/kg) }\end{array}$ \\
\hline A14-CR02-109 & CR02 & 109 & duplicate & $5 / 19 / 2003$ & 6/2/2003 & 0.000 & 0.000 & 0.000 & 0.000 & 0.000 \\
\hline A14-CR02-111 & CR02 & 111 & sample & $5 / 19 / 2003$ & $5 / 22 / 2003$ & 66.898 & 1.069 & 0.000 & 0.003 & 0.000 \\
\hline A14-CR02-111 & CR02 & 111 & duplicate & $5 / 19 / 2003$ & $6 / 2 / 2003$ & 20.768 & 0.615 & 0.000 & 0.002 & 0.000 \\
\hline A14-CR02-112 & CR02 & 112 & sample & $5 / 19 / 2003$ & $5 / 22 / 2003$ & 2.141 & 0.121 & 0.000 & 0.000 & 0.000 \\
\hline A14-CR02-112 & CR02 & 112 & duplicate & $5 / 19 / 2003$ & $6 / 2 / 2003$ & 1.760 & 0.103 & 0.000 & 0.000 & 0.000 \\
\hline A14-CR02-114 & CR02 & 114 & sample & $5 / 19 / 2003$ & $5 / 22 / 2003$ & 0.363 & 0.028 & 0.000 & 0.000 & 0.000 \\
\hline A14-CR02-114 & CR02 & 114 & duplicate & $5 / 19 / 2003$ & $6 / 2 / 2003$ & 0.738 & 0.043 & 0.000 & 0.000 & 0.000 \\
\hline A14-CR02-115 & CR02 & 115 & sample & $5 / 19 / 2003$ & $5 / 22 / 2003$ & 0.030 & 0.005 & 0.000 & 0.000 & 0.000 \\
\hline A14-CR02-115 & CR02 & 115 & duplicate & $5 / 19 / 2003$ & $6 / 2 / 2003$ & 0.046 & 0.011 & 0.000 & 0.000 & 0.000 \\
\hline A14-CR02-117 & CR02 & 117 & sample & $5 / 19 / 2003$ & $5 / 22 / 2003$ & 0.085 & 0.009 & 0.000 & 0.000 & 0.000 \\
\hline A14-CR02-117 & CR02 & 117 & duplicate & $5 / 19 / 2003$ & $6 / 2 / 2003$ & 0.019 & 0.000 & 0.000 & 0.000 & 0.000 \\
\hline A14-CR02-118 & CR02 & 118 & sample & $5 / 19 / 2003$ & $5 / 22 / 2003$ & 2.824 & 0.101 & 0.000 & 0.000 & 0.000 \\
\hline A14-CR02-118 & CR02 & 118 & duplicate & $5 / 19 / 2003$ & $6 / 2 / 2003$ & 6.339 & 0.220 & 0.000 & 0.000 & 0.000 \\
\hline
\end{tabular}


Table 8 - Sediment Analysis Results for Boring CR03

\begin{tabular}{|c|c|c|c|c|c|c|c|c|c|c|}
\hline Sample ID & Boring & Depth $(\mathrm{ft})$ & Type & $\begin{array}{c}\text { Date } \\
\text { Collected }\end{array}$ & $\begin{array}{c}\text { Date } \\
\text { Analyzed }\end{array}$ & $\begin{array}{c}\text { PCE } \\
(\mathrm{mg} / \mathrm{kg})\end{array}$ & $\begin{array}{c}\text { TCE } \\
(\mathrm{mg} / \mathrm{kg})\end{array}$ & $\begin{array}{c}\text { C-DCE } \\
(\mathrm{mg} / \mathrm{kg})\end{array}$ & $\begin{array}{c}\text { TCA } \\
(\mathrm{mg} / \mathrm{kg})\end{array}$ & $\begin{array}{l}11 \mathrm{DCE} \\
(\mathrm{mg} / \mathrm{kg})\end{array}$ \\
\hline A14-CR03-001 & R03 & 1 & sample & $5 / 20 / 2003$ & $5 / 26 / 2003$ & 0.035 & 0.000 & 0.000 & 0.000 & 0.000 \\
\hline A14-CR03-001 & CR03 & 1 & duplicate & $5 / 20 / 2003$ & $7 / 14 / 2003$ & 0.032 & 0.000 & 0.000 & 0.000 & \\
\hline A14-CR03-002 & CR03 & 2 & sample & $5 / 20 / 2003$ & $5 / 26 / 2003$ & 0.094 & .002 & 0.000 & 0.000 & 000 \\
\hline A14-CR03-002 & CR03 & 2 & duplicate & $5 / 20 / 2003$ & $7 / 14 / 2003$ & 0.165 & 0.003 & 0.000 & 0.000 & 00 \\
\hline A14-CR03-003 & CR03 & 3 & sample & $5 / 20 / 2003$ & $5 / 26 / 2003$ & 0.109 & 0.005 & 0.000 & 0.000 & 0.000 \\
\hline A14-CR03-003 & CR03 & 3 & duplicate & $5 / 20 / 2003$ & $7 / 14 / 2003$ & 0.133 & 0.006 & 0.000 & 0.000 & 0.000 \\
\hline A14-CR03-004 & CR03 & 4 & sample & $5 / 20 / 2003$ & $5 / 26 / 2003$ & 0.369 & 0.016 & 0.000 & 0.000 & \\
\hline $\mathrm{A} 14-\mathrm{C}$ & CR03 & 4 & duplicate & $5 / 20 / 2003$ & $7 / 14 / 2003$ & 0.489 & .033 & 0.000 & 0.000 & \\
\hline A14-C & CR03 & 5 & sample & $5 / 20 / 2003$ & $5 / 26 / 2003$ & 0.630 & 0.024 & 0.000 & 0.000 & 00 \\
\hline A14-CR03-005 & CR03 & 5 & duplicate & $5 / 20 / 2003$ & $7 / 14 / 2003$ & 0.764 & 0.038 & 0.000 & 0.000 & 000 \\
\hline A14-CR03-006 & CR03 & 6 & & $5 / 20 / 2003$ & $5 / 26 / t$ & 2.848 & 0.048 & 0.000 & 0.000 & \\
\hline A14-C & CR03 & 6 & duplicate & $5 / 20 / 5$ & $7 / 14 / 2003$ & 1.447 & 0.041 & 0.000 & 0.003 & \\
\hline A14-C & CR03 & 7 & samp & $5 / 20$ & $5 / 26 / 2003$ & 31.969 & 0.116 & 0.000 & 0.008 & \\
\hline A14-C & CR03 & 7 & duplic & $5 / 20 / 2$ & $7 / 14 / 2003$ & 9.744 & 0.051 & 0.000 & 0.010 & 00 \\
\hline A14-C & CR03 & 8 & sam & $5 / 20 / 2$ & $5 / 26 / 2003$ & 1258.498 & 6.897 & 19 & 0.931 & 00 \\
\hline $\mathrm{A} 14-\mathrm{C}$ & CR03 & 8 & duplicate & $5 / 20 / 2$ & $7 / 14 / 2003$ & 777.509 & 4.331 & & 1.346 & 00 \\
\hline A14-C & $\overline{\mathrm{CR}}$ & 9 & sam & $5 / 20 /$ & $5 / 26 / 2003$ & 1474.970 & 15.336 & 39 & 1.868 & \\
\hline A14-C & CR03 & 9 & duplicate & $5 / 20 /$ & $7 / 14 / 2003$ & 1039.801 & 13.364 & 0.239 & 2.626 & 000 \\
\hline A14-C & CR03 & 10 & sample & $5 / 20 / 2$ & $5 / 26 / 2003$ & 1628.262 & 24.412 & 0.181 & 2.286 & 00 \\
\hline A14-C & CR03 & 10 & duplicate & $5 / 20 / 2003$ & $7 / 14 / 2003$ & 1236.636 & 19.848 & 0.262 & 3.070 & 0.000 \\
\hline A14-C & CR03 & & & $5 / 20 / 2003$ & $5 / 26 / 2003$ & 2981.095 & 63.077 & 0.227 & 4.684 & \\
\hline$\overline{A 14-C}$ & & & duplicate & $5 / 20 /$ & $7 / 14 / 2003$ & 4008.918 & 100.527 & & 8.895 & \\
\hline A14-C & CR03 & 12 & sample & $5 / 20 / 2003$ & $5 / 26 / 2003$ & 2519.351 & 103.680 & 0.395 & 7.744 & 0.000 \\
\hline $\mathrm{A} 14-\mathrm{CF}$ & CR03 & 12 & duplicate & $5 / 20 / 2003$ & $7 / 14 / 2003$ & 1544.491 & 101.746 & 2.215 & 11.423 & 0.000 \\
\hline A14-C & CR03 & 13 & sam & $5 / 20 / 2003$ & $5 / 26 / 2003$ & 2488.545 & 163.737 & 0.319 & 8.737 & 0.000 \\
\hline A14-C & CR03 & 13 & duplicate & $5 / 20 / 2003$ & $7 / 14 / 2003$ & 875.915 & 100.376 & 0.615 & 8.965 & 0.000 \\
\hline A14-CR03-014 & & 14 & sample & $5 / 20 / 2003$ & $5 / 26 / 2003$ & 2061.590 & 195.548 & 0.284 & 6.500 & 0.000 \\
\hline A14-CR03-014 & CR03 & 14 & duplicate & $5 / 20 / 2003$ & $7 / 14 / 2003$ & 1295.827 & 178.683 & 0.671 & 8.346 & 0.000 \\
\hline
\end{tabular}




\begin{tabular}{|c|c|c|c|c|c|c|c|c|c|c|}
\hline Sample ID & Boring & Depth (ft) & Type & $\begin{array}{c}\text { Date } \\
\text { Collected }\end{array}$ & $\begin{array}{c}\text { Date } \\
\text { Analyzed }\end{array}$ & $\begin{array}{c}\text { PCE } \\
(\mathrm{mg} / \mathrm{kg})\end{array}$ & $\begin{array}{c}\text { TCE } \\
(\mathrm{mg} / \mathrm{kg})\end{array}$ & $\begin{array}{l}\text { C-DCE } \\
(\mathrm{mg} / \mathrm{kg})\end{array}$ & $\begin{array}{c}\text { TCA } \\
(\mathrm{mg} / \mathrm{kg})\end{array}$ & $\begin{array}{l}\text { 11DCE } \\
(\mathrm{mg} / \mathrm{kg})\end{array}$ \\
\hline A14-CR03-015 & R03 & 15 & sample & $5 / 20 / 2003$ & $5 / 26 / 2003$ & 2865.756 & 280.619 & 0.361 & 7.615 & 0.000 \\
\hline A14-CR03-015 & CR03 & 15 & duplicate & $5 / 20 / 2003$ & $7 / 14 / 2003$ & 1435.427 & 254.922 & 0.772 & 9.901 & .000 \\
\hline A14-CR03-016 & CR03 & 16 & sample & $5 / 20 / 2003$ & $5 / 26 / 2003$ & 539.419 & 243.470 & 0.193 & 5.255 & .000 \\
\hline A14-CR03-016 & $\overline{\mathrm{CR} 03}$ & 16 & & $5 / 20 / 2003$ & $7 / 14 / 2003$ & 1504.223 & & 0.664 & & \\
\hline $\mathrm{A} 14-\mathrm{C}$ & CR03 & 17 & sample & $5 / 20 / 2003$ & $5 / 26 / 2003$ & 1694.941 & 204.563 & 0.321 & 6.315 & 00 \\
\hline A14-CR03-017 & CR03 & 17 & duplicate & $5 / 20 / 2003$ & $7 / 14 / 2003$ & 1279.017 & 155.654 & 0.780 & 7.209 & 0.000 \\
\hline A14-CR03-018 & CR03 & 18 & sample & $5 / 20 / 2003$ & $5 / 26 / 2003$ & 4769.711 & 342.668 & 0.399 & 7.875 & 0.000 \\
\hline A14-CR03-018 & CR03 & 18 & duplicate & $5 / 20 / 2003$ & $7 / 14 / 2003$ & 1038.897 & 175.284 & 0.849 & 5.755 & 0.000 \\
\hline A14-C & CR03 & 19 & sample & $5 / 20 / 2003$ & $5 / 26 / 2003$ & 3415.767 & 210.831 & 0.338 & 1.293 & \\
\hline A14- & CR03 & 19 & duplic & $5 / 20$ & $7 / 14 / 2003$ & 1556.304 & 166.244 & 0.749 & 1.619 & \\
\hline A14-C & CR03 & 20 & samp & $5 / 20 / 2003$ & $5 / 26 / 2003$ & 2307.342 & 107.890 & 0.140 & 0.163 & 0.000 \\
\hline A14-C & CR03 & 20 & duplic & $5 / 20 / 2003$ & $7 / 14 / 2003$ & 1095.009 & 82.246 & 0.290 & 0.242 & 0.000 \\
\hline A14-C & CR03 & 21 & sam & $5 / 20 / 2003$ & $5 / 26 / 2003$ & 3003.373 & 121.225 & 0.090 & 0.185 & .000 \\
\hline 21 & CR03 & 21 & dupli & $5 / 20 /$ & $7 / 14 / 2003$ & 875.758 & 55.873 & 0.000 & 0.156 & 00 \\
\hline A14-C & CR03 & 22 & samp & $5 / 20 / 2003$ & $5 / 26 / 2003$ & 1996.035 & 46.999 & 0.000 & 0.031 & \\
\hline A14-C & CR03 & 22 & duplic & $5 / 20 / 2003$ & $7 / 14 / 2003$ & 1503.645 & 53.842 & 0.000 & 0.083 & 0.000 \\
\hline A14-C & CR03 & 23 & & $5 / 20 /$ & $5 / 26 / 20$ & 1618.5 & 44.885 & & & 00 \\
\hline$\overline{A 14-C}$ & CR03 & 23 & duplicate & $5 / 20 / 2003$ & $7 / 14 / 2003$ & 954.105 & 39.741 & 0.000 & 0.057 & 00 \\
\hline A14-C & CR03 & 24 & sam & $5 / 20 /$ & $5 / 26 / 2003$ & 1314.959 & 26.209 & 0.000 & 0.016 & 000 \\
\hline A14-C & CR03 & 24 & dupli & $5 / 20$ & $7 / 14 / 20$ & 826.368 & 13.998 & 0.000 & 0.018 & 00 \\
\hline A14-C & CR03 & 25 & sam & $5 / 20 / 2003$ & $5 / 26 / 2003$ & 2965.145 & 31.641 & 0.000 & 0.042 & 00 \\
\hline A14-C & CR03 & 25 & dupli & $5 / 20 / 2003$ & $7 / 14 / 2003$ & 1137.433 & 23. & 0.000 & & 00 \\
\hline A14-C & & 26 & & $5 / 20 /$ & $5 / 26 / 20$ & 1779.698 & 15.908 & & & \\
\hline A14-C & CR03 & 26 & duplicate & $5 / 20 / 2003$ & $7 / 14 / 2003$ & 1264.150 & 15.040 & 0.000 & 0.021 & 000 \\
\hline A14-C & CR03 & 27 & sam & $5 / 21 / 2003$ & $5 / 26 / 2003$ & 2967.026 & 8.685 & 0.000 & 0.008 & 0.000 \\
\hline A14-C & CR03 & 27 & dupli & $5 / 21 / 2003$ & $7 / 14 / 2003$ & 1488.524 & 4.203 & 0.000 & 0.010 & 0.000 \\
\hline A14- & & 29 & sam & $5 / 21 /$ & $5 / 26 / 2003$ & 751.048 & 25.591 & 0.199 & 0.235 & 0.000 \\
\hline & & 29 & duplicate & $5 / 21 / 2003$ & $7 / 14 / 2003$ & 831.024 & 26.303 & 0.452 & 0.376 & \\
\hline A14-C & & 30 & sample & $5 / 21 / 2003$ & $5 / 26 / 2003$ & 1073.209 & 26.319 & 0.078 & 0.388 & 0.000 \\
\hline A14-CR03-030 & CR03 & 30 & duplicate & $5 / 21 / 2003$ & $7 / 14 / 2003$ & 892.260 & 20.044 & 0.207 & 0.423 & 0.000 \\
\hline A14-CR03-035 & CR03 & 35 & sample & $5 / 21 / 2003$ & $5 / 26 / 2003$ & 1.729 & 0.012 & 0.000 & 0.000 & 0.000 \\
\hline
\end{tabular}




\begin{tabular}{|c|c|c|c|c|c|c|c|c|c|c|}
\hline Sample ID & Boring & Depth (ft) & Type & $\begin{array}{c}\text { Date } \\
\text { Collected }\end{array}$ & $\begin{array}{c}\text { Date } \\
\text { Analyzed }\end{array}$ & $\begin{array}{c}\text { PCE } \\
(\mathrm{mg} / \mathrm{kg})\end{array}$ & $\begin{array}{c}\text { TCE } \\
(\mathrm{mg} / \mathrm{kg})\end{array}$ & $\begin{array}{l}\text { C-DCE } \\
(\mathrm{mg} / \mathrm{kg})\end{array}$ & $\begin{array}{c}\text { TCA } \\
(\mathrm{mg} / \mathrm{kg})\end{array}$ & $\begin{array}{l}\text { 11DCE } \\
(\mathrm{mg} / \mathrm{kg})\end{array}$ \\
\hline A14-CR03-035 & R03 & 35 & duplicate & $5 / 21 / 2003$ & $7 / 14 / 2003$ & 8.769 & 0.117 & 0.000 & 0.005 & 0.000 \\
\hline A14-CR03-036 & CR03 & 36 & sample & $5 / 21 / 2003$ & $5 / 26 / 2003$ & 1.294 & 0.006 & 0.000 & 0.000 & 0.000 \\
\hline A14-CR03-036 & CR03 & 36 & duplicate & $5 / 21 / 2003$ & $7 / 14 / 2003$ & 1.315 & 0.018 & 0.000 & 0.000 & 0.000 \\
\hline A14-CR03-037 & CR03 & 37 & & $5 / 21 / 2003$ & $5 / 26 / 2003$ & 1.363 & 0.014 & & & \\
\hline A14-CR03-037 & CR03 & 37 & duplicate & $5 / 21 / 2003$ & $7 / 14 / 2003$ & 0.816 & 0.028 & 0.000 & 0.000 & .000 \\
\hline A14-CR03-038 & CR03 & 38 & sample & $5 / 21 / 2003$ & $5 / 26 / 2003$ & 10.832 & 0.008 & 0.000 & 0.000 & 0.000 \\
\hline A14-CR03-038 & CR03 & 38 & duplicate & $5 / 21 / 2003$ & $7 / 14 / 2003$ & 3.607 & 0.015 & 0.000 & 0.000 & 0.000 \\
\hline A14-CR03-039 & CR03 & 39 & sample & $5 / 21 / 2003$ & $5 / 26 / 2003$ & 4.394 & 0.006 & 0.000 & 0.000 & 0.000 \\
\hline A14-CR03-039 & CR03 & 39 & duplicate & $5 / 21 / 2003$ & $7 / 14 / 2003$ & 4.394 & 0.026 & 0.000 & 0.000 & 000 \\
\hline$\overline{A 14-C}$ & CR03 & 40 & sample & $5 / 21 / 2003$ & $5 / 26 / 2003$ & 3.634 & 0.011 & 0.000 & 0.000 & 00 \\
\hline A14-CR03-040 & CR03 & 40 & duplicate & $5 / 21 / 2003$ & $7 / 14 / 2003$ & 2.646 & 0.006 & 0.000 & 0.000 & 0.000 \\
\hline A14-CR03-041 & CR03 & 41 & sample & $5 / 21 / 2003$ & $5 / 26 / 2003$ & 3.061 & 0.012 & 0.000 & 0.000 & 0.000 \\
\hline A14-CR03-041 & CR03 & 41 & duplicate & $5 / 21 / 2003$ & $7 / 14 / 2003$ & 2.278 & 0.012 & 0.000 & 0.000 & 0.000 \\
\hline A14-C & CR03 & 42 & sample & $5 / 21 / 2003$ & $5 / 26 / 2003$ & 0.121 & 0.004 & 00 & 00 & \\
\hline $\mathrm{A} 14-\mathrm{C}$ & CR03 & 42 & duplicate & $5 / 21 / 2003$ & $7 / 14 / 2003$ & 0.360 & 0.012 & 0.000 & 0.000 & 0.000 \\
\hline A14-C & CR03 & 43 & sample & $5 / 21 / 2003$ & $5 / 26 / 2003$ & 0.098 & 0.003 & 0.000 & 0.000 & 0.000 \\
\hline A14-C & CR03 & 43 & duplicate & $5 / 21 / 2003$ & $7 / 14 / 2003$ & 0.242 & 0.005 & 0.000 & 0.000 & 00 \\
\hline $\mathrm{A} 14-\mathrm{C}$ & CR03 & 44 & sample & $5 / 21 / 2003$ & $5 / 26 / 2003$ & 0.059 & 0.003 & 0.000 & 0.000 & 0.000 \\
\hline $\mathrm{A} 14-\mathrm{C}$ & CR03 & 44 & duplicate & $5 / 21 / 2003$ & $7 / 14 / 2003$ & 0.155 & 0.003 & 0.000 & 0.000 & 0.000 \\
\hline A14-C & CR03 & 45 & sample & $5 / 21 / 2003$ & $5 / 26 / 2003$ & 0.050 & 0.003 & 0.000 & 0.000 & 0.000 \\
\hline A14-CR03-045 & CR03 & 45 & duplicate & $5 / 21 / 2003$ & $7 / 14 / 2003$ & 0.258 & 09 & 0.000 & 0.000 & 0.000 \\
\hline $\mathrm{A} 14-\mathrm{C}$ & CR03 & 46 & sample & $5 / 21 / 2003$ & $5 / 26 / 2003$ & 2.072 & 0.042 & 0.000 & 0.000 & 0.000 \\
\hline$\overline{A 14-C}$ & CR03 & 46 & duplicate & $5 / 21 / 2003$ & $7 / 14 / 2003$ & 1.846 & 0.028 & 00 & 0.000 & 00 \\
\hline A14-C & CR03 & 47 & sample & $5 / 21 / 2003$ & $5 / 26 / 2003$ & 2.478 & 0.028 & 0.000 & 0.000 & 0.000 \\
\hline $\mathrm{A} 14-\mathrm{C}$ & CR03 & 47 & duplicate & $5 / 21 / 2003$ & $7 / 14 / 2003$ & 1.672 & 0.031 & 0.000 & 0.000 & 0.000 \\
\hline A14-C & CR03 & 48 & sample & $5 / 21 / 2003$ & $5 / 26 / 2003$ & 1.727 & 0.020 & 0.000 & 0.000 & 0.000 \\
\hline A14-C & CR03 & 48 & duplicate & $5 / 21 / 2003$ & $7 / 14 / 2003$ & 2.397 & 0.048 & 0.000 & 0.000 & 0.000 \\
\hline A14-CR & CR03 & 50 & sample & $5 / 21 / 2003$ & $5 / 26 / 2003$ & 1.026 & & 0.000 & 0.000 & 0.000 \\
\hline A14-CR03-050 & CR03 & 50 & duplicate & $5 / 21 / 2003$ & $7 / 14 / 2003$ & 0.349 & 0.008 & 0.000 & 0.000 & 0.000 \\
\hline A14-CR03-051 & CR03 & 51 & sample & $5 / 21 / 2003$ & $5 / 26 / 2003$ & 0.825 & 0.009 & 0.000 & 0.000 & 0.000 \\
\hline A14-CR03-051 & CR03 & 51 & duplicate & $5 / 21 / 2003$ & $7 / 14 / 2003$ & 0.578 & 0.016 & 0.000 & 0.000 & 0.000 \\
\hline
\end{tabular}


WSRC-TR-2003-00540

September 2003

\begin{tabular}{|c|c|c|c|c|c|c|c|c|c|c|}
\hline Sample ID & Boring & Depth (ft) & Type & $\begin{array}{c}\text { Date } \\
\text { Collected }\end{array}$ & $\begin{array}{c}\text { Date } \\
\text { Analyzed }\end{array}$ & $\begin{array}{c}\text { PCE } \\
(\mathrm{mg} / \mathrm{kg})\end{array}$ & $\begin{array}{c}\text { TCE } \\
(\mathrm{mg} / \mathrm{kg})\end{array}$ & $\begin{array}{l}\text { C-DCE } \\
(\mathrm{mg} / \mathrm{kg})\end{array}$ & $\begin{array}{c}\text { TCA } \\
(\mathrm{mg} / \mathrm{kg}) \\
\end{array}$ & $\begin{array}{l}\text { 11DCE } \\
(\mathrm{mg} / \mathrm{kg})\end{array}$ \\
\hline A14-CR03-059 & R03 & 59 & sample & $5 / 21 / 2003$ & $5 / 27 / 2003$ & 0.000 & 11.375 & 0.096 & 0.441 & 0.000 \\
\hline A14-CR03-059 & CR03 & 59 & duplicate & $5 / 21 / 2003$ & $6 / 4 / 2003$ & 1234.711 & 38.866 & 0.138 & 1.209 & 0.000 \\
\hline A14-CR03-060 & CR03 & 60 & & $5 / 21 / 2003$ & $5 / 27 / 2003$ & 15.596 & 0.060 & 0.000 & 0.000 & 0.000 \\
\hline A14-CR03-060 & CR03 & 60 & duplicate & $5 / 21 / 2003$ & $6 / 4 / 2003$ & 14.676 & 0.067 & 0.000 & 0.000 & 0.000 \\
\hline A14-CR03-062 & CR03 & 62 & sample & $5 / 21 / 2003$ & $5 / 27 / 2003$ & 7.363 & 0.262 & 000 & 0.009 & .000 \\
\hline A14-CR03-062 & CR03 & 62 & duplicate & $5 / 21 / 2003$ & $6 / 4 / 2003$ & 9.095 & 0.311 & 0.000 & 0.007 & 0.000 \\
\hline A14-CR03-063 & CR03 & 63 & sample & $5 / 21 / 2003$ & $5 / 27 / 2003$ & 0.216 & 0.010 & 0.000 & 0.000 & 0.000 \\
\hline A14-CR03-063 & CR03 & 63 & duplicate & $5 / 21 / 2003$ & $6 / 4 / 2003$ & 0.434 & 0.013 & 0.000 & 0.000 & 0.000 \\
\hline A14-CR03-065 & CR03 & 65 & sample & $5 / 21 / 2003$ & $5 / 27 / 2003$ & 0.466 & 0.023 & 0.000 & 0.000 & .000 \\
\hline $\mathrm{A} 14-\mathrm{CP}$ & CR03 & 65 & duplicate & $5 / 21 / 2003$ & $6 / 4 / 2003$ & 0.245 & 0.008 & 0.000 & 0.000 & 0.000 \\
\hline A14-CR03-066 & CR03 & 66 & sample & $5 / 21 / 2003$ & $5 / 27 / 2003$ & 0.091 & 0.000 & 0.000 & 0.000 & 0.000 \\
\hline A14-CR03-066 & CR03 & 66 & duplicate & $5 / 21 / 2003$ & $6 / 4 / 2003$ & 0.196 & 0.007 & 0.000 & 0.000 & 0.000 \\
\hline A14-CRC & CR03 & 68 & sam & $5 / 21 / 2003$ & $5 / 27 / 2003$ & 0.406 & 0.015 & 0.000 & 0.000 & 0.000 \\
\hline $\mathrm{A} 14-\mathrm{C}$ & CR03 & 68 & dupli & $5 / 21 / 2003$ & $6 / 4 / 2003$ & 0.132 & 0.004 & 0.000 & 0.000 & 0.000 \\
\hline A14-CR03-071 & CR03 & 71 & sample & $5 / 21 / 2003$ & $5 / 27 / 2003$ & 18.412 & 0.250 & 0.000 & 0.007 & 0.000 \\
\hline A14-CR03-071 & CR03 & 71 & duplicate & $5 / 21 / 2003$ & $6 / 4 / 2003$ & 77.801 & 0.900 & 0.000 & 0.014 & 0.000 \\
\hline A14-CR03-072 & CR03 & 72 & sample & $5 / 21 / 2003$ & $5 / 27 / 2003$ & 0.059 & 0.000 & 0.000 & 0.000 & 0.000 \\
\hline A14-CR03 & CR03 & 72 & duplicate & $5 / 21 / 2003$ & $6 / 4 / 2003$ & 0.128 & 0.000 & 0.000 & 0.000 & 0.000 \\
\hline A14-CR03-075 & CR03 & 75 & sample & $5 / 21 / 2003$ & $5 / 27 / 2003$ & 0.035 & 0.000 & 0.000 & 0.000 & 0.000 \\
\hline A14-CR03-075 & CR03 & 75 & duplicate & $5 / 21 / 2003$ & $6 / 4 / 2003$ & 0.038 & 0.000 & 0.000 & 0.000 & 0.000 \\
\hline A14-CR03-078 & CR03 & 78 & sample & $5 / 21 / 2003$ & $5 / 27 / 2003$ & 0.154 & 0.005 & 0.000 & 0.000 & 0.000 \\
\hline A14-CR03-078 & CR03 & 78 & duplicate & $5 / 21 / 2003$ & $6 / 4 / 2003$ & 0.148 & 0.003 & 0.000 & 0.000 & 0.000 \\
\hline
\end{tabular}


Table 9 - Sediment Analysis Results for Boring CR04

\begin{tabular}{|c|c|c|c|c|c|c|c|c|c|c|}
\hline Sample ID & Boring & Depth (ft) & Type & $\begin{array}{c}\text { Date } \\
\text { Collected }\end{array}$ & $\begin{array}{c}\text { Date } \\
\text { Analyzed }\end{array}$ & $\begin{array}{c}\text { PCE } \\
(\mathrm{mg} / \mathrm{kg})\end{array}$ & $\begin{array}{c}\text { TCE } \\
(\mathrm{mg} / \mathrm{kg})\end{array}$ & $\begin{array}{l}\text { C-DCE } \\
(\mathrm{mg} / \mathrm{kg})\end{array}$ & $\begin{array}{c}\text { TCA } \\
(\mathrm{mg} / \mathrm{kg})\end{array}$ & $\begin{array}{l}11 \mathrm{DCE} \\
(\mathrm{mg} / \mathrm{kg})\end{array}$ \\
\hline A14-CR04-001 & CR04 & 1 & sample & $5 / 22 / 2003$ & $5 / 27 / 2003$ & 0.000 & 0.000 & 0.000 & 0.000 & 0.000 \\
\hline A14-CR04-001 & CR04 & 1 & duplicate & $5 / 22 / 2003$ & $6 / 4 / 2003$ & 0.000 & 0.000 & 0.000 & 0.000 & 0.000 \\
\hline A14-CR04-002 & CR04 & 2 & sample & $5 / 22 / 2003$ & $5 / 27 / 2003$ & 0.000 & 0.000 & 0.000 & 0.000 & 0.000 \\
\hline A14-CR04-002 & CR04 & 2 & duplicate & $5 / 22 / 2003$ & $6 / 4 / 2003$ & 0.005 & 0.000 & 0.000 & 0.000 & 0.000 \\
\hline A14-CR04-003 & CR04 & 3 & sample & $5 / 22 / 2003$ & $5 / 27 / 2003$ & 0.003 & 0.000 & 0.000 & 0.000 & 0.000 \\
\hline A14-CR04-003 & CR04 & 3 & duplicate & $5 / 22 / 2003$ & $6 / 4 / 2003$ & 0.009 & 0.000 & 0.000 & 0.000 & 0.000 \\
\hline A14-CR04-004 & CR04 & 4 & sample & $5 / 22 / 2003$ & $5 / 27 / 2003$ & 0.012 & 0.000 & 0.000 & 0.000 & 0.000 \\
\hline A14-CR04-004 & CR04 & 4 & duplicate & $5 / 22 / 2003$ & $6 / 4 / 2003$ & 0.024 & 0.000 & 0.000 & 0.000 & 0.000 \\
\hline A14-CR04-005 & CR04 & 5 & sample & $5 / 22 / 2003$ & $5 / 27 / 2003$ & 0.031 & 0.000 & 0.000 & 0.000 & 0.000 \\
\hline A14-CR04-005 & CR04 & 5 & duplicate & $5 / 22 / 2003$ & $6 / 4 / 2003$ & 0.042 & 0.000 & 0.000 & 0.000 & 0.000 \\
\hline A14-CR04-006 & CR04 & 6 & sample & $5 / 22 / 2003$ & $5 / 27 / 2003$ & 0.011 & 0.000 & 0.000 & 0.000 & 0.000 \\
\hline A14-CR04-006 & CR04 & 6 & duplicate & $5 / 22 / 2003$ & $6 / 4 / 2003$ & 0.020 & 0.000 & 0.000 & 0.000 & 0.000 \\
\hline A14-CR04-007 & CR04 & 7 & sample & $5 / 22 / 2003$ & $5 / 27 / 2003$ & 0.000 & 0.000 & 0.000 & 0.000 & 0.000 \\
\hline A14-CR04-007 & CR04 & 7 & duplicate & $5 / 22 / 2003$ & $6 / 4 / 2003$ & 0.004 & 0.000 & 0.000 & 0.000 & 0.000 \\
\hline A14-CR04-008 & CR04 & 8 & sample & $5 / 22 / 2003$ & $5 / 27 / 2003$ & 0.004 & 0.000 & 0.000 & 0.000 & 0.000 \\
\hline A14-CR04-008 & CR04 & 8 & duplicate & $5 / 22 / 2003$ & $6 / 4 / 2003$ & 0.008 & 0.000 & 0.000 & 0.000 & 0.000 \\
\hline A14-CR04-009 & CR04 & 9 & sample & $5 / 22 / 2003$ & $5 / 27 / 2003$ & 0.011 & 0.000 & 0.000 & 0.000 & 0.000 \\
\hline A14-CR04-009 & CR04 & 9 & duplicate & $5 / 22 / 2003$ & $6 / 4 / 2003$ & 0.014 & 0.000 & 0.000 & 0.000 & 0.000 \\
\hline A14-CR04-010 & CR04 & 10 & sample & $5 / 22 / 2003$ & $5 / 27 / 2003$ & 0.063 & 0.000 & 0.000 & 0.000 & 0.000 \\
\hline A14-CR04-010 & CR04 & 10 & duplicate & $5 / 22 / 2003$ & $6 / 4 / 2003$ & 0.039 & 0.000 & 0.000 & 0.000 & 0.000 \\
\hline A14-CR04-011 & CR04 & 11 & sample & $5 / 22 / 2003$ & $5 / 27 / 2003$ & 0.067 & 0.000 & 0.000 & 0.000 & 0.000 \\
\hline A14-CR04-011 & CR04 & 11 & duplicate & $5 / 22 / 2003$ & $6 / 4 / 2003$ & 0.047 & 0.000 & 0.000 & 0.000 & 0.000 \\
\hline A14-CR04-012 & CR04 & 12 & sample & $5 / 22 / 2003$ & $5 / 27 / 2003$ & 0.097 & 0.000 & 0.000 & 0.000 & 0.000 \\
\hline A14-CR04-012 & CR04 & 12 & duplicate & $5 / 22 / 2003$ & 6/4/2003 & 0.050 & 0.000 & 0.000 & 0.000 & 0.000 \\
\hline A14-CR04-013 & CR04 & 13 & sample & $5 / 22 / 2003$ & $5 / 27 / 2003$ & 0.056 & 0.000 & 0.000 & 0.000 & 0.000 \\
\hline A14-CR04-013 & CR04 & 13 & duplicate & $5 / 22 / 2003$ & $6 / 4 / 2003$ & 0.025 & 0.000 & 0.000 & 0.000 & 0.000 \\
\hline A14-CR04-014 & CR04 & 14 & sample & $5 / 22 / 2003$ & $5 / 27 / 2003$ & 0.554 & 0.000 & 0.201 & 0.000 & 0.000 \\
\hline A14-CR04-014 & CR04 & 14 & duplicate & $5 / 22 / 2003$ & $6 / 4 / 2003$ & 0.478 & 0.014 & 0.100 & 0.000 & 0.000 \\
\hline
\end{tabular}




\begin{tabular}{|c|c|c|c|c|c|c|c|c|c|c|}
\hline Sample ID & Boring & Depth (ft) & Type & $\begin{array}{c}\text { Date } \\
\text { Collected }\end{array}$ & $\begin{array}{c}\text { Date } \\
\text { Analyzed }\end{array}$ & $\begin{array}{c}\text { PCE } \\
(\mathrm{mg} / \mathrm{kg})\end{array}$ & $\begin{array}{c}\text { TCE } \\
(\mathrm{mg} / \mathrm{kg})\end{array}$ & $\begin{array}{l}\text { C-DCE } \\
(\mathrm{mg} / \mathrm{kg})\end{array}$ & $\begin{array}{c}\text { TCA } \\
(\mathrm{mg} / \mathrm{kg})\end{array}$ & $\begin{array}{l}\text { 11DCE } \\
(\mathrm{mg} / \mathrm{kg})\end{array}$ \\
\hline A14-CR04-015 & CR04 & 15 & sample & $5 / 22 / 2003$ & $5 / 27 / 2003$ & 0.080 & 0.000 & 0.000 & 0.000 & 0.000 \\
\hline A14-CR04-015 & $\overline{\mathrm{R} 04}$ & 15 & duplicate & $5 / 22 / 2003$ & $6 / 4 / 2003$ & .072 & .004 & 000 & .000 & .000 \\
\hline A14-CR04-016 & CR04 & 16 & sample & $5 / 22 / 2003$ & $5 / 27 / 2003$ & 0.101 & 0.000 & 0.000 & 0.000 & 0.000 \\
\hline A14-CR04-016 & CR04 & 16 & duplicate & $5 / 22 / 2003$ & $6 / 4 / 2003$ & 0.029 & 0.000 & 0.000 & 0.000 & 0.000 \\
\hline 14-CR04-018 & $\overline{R 04}$ & 18 & sample & $5 / 22 / 2003$ & $5 / 27 / 2003$ & .041 & 00 & .000 & 0.000 & \\
\hline R04-018 & 204 & 18 & duplicate & $5 / 22 / 2003$ & $6 / 4 / 2003$ & 071 & 03 & 00 & .000 & .000 \\
\hline R04-019 & 204 & 19 & sample & $5 / 22 / 2003$ & $5 / 27 / 2003$ & 026 & 00 & 00 & 0.000 & .000 \\
\hline A14-CR04-019 & CR04 & 19 & duplic & $5 / 22 / 2003$ & $6 / 4 / 2003$ & 0.057 & 0.003 & 0.000 & 0.000 & 0.000 \\
\hline A14-CR04-020 & CR04 & 20 & sample & $5 / 22 / 2003$ & $5 / 27 / 2003$ & 0.015 & 0.000 & 0.000 & 0.000 & 0.000 \\
\hline 14-CR04-020 & R04 & 20 & dupli & $5 / 22 / 20$ & $6 / 4 / 2003$ & & 00 & .000 & 0.000 & \\
\hline $4-021$ & 34 & 21 & sam & $5 / 22 / 20$ & $5 / 27 / 2003$ & & & 00 & .000 & \\
\hline A14-CR04-021 & CR04 & 21 & duplicate & $5 / 22 / 2003$ & $6 / 4 / 2003$ & 0.017 & 000 & 0.000 & 0.000 & 0.000 \\
\hline A14-CR04-022 & CR04 & 22 & sample & $5 / 22 / 2003$ & $5 / 27 / 2003$ & 0.042 & 0.000 & 0.000 & 0.000 & 0.000 \\
\hline 14-CR04-022 & CR04 & 22 & duplicate & $5 / 22 / 2003$ & $6 / 4 / 2003$ & 0.048 & 0.003 & 0.000 & 0.000 & 0.000 \\
\hline R04-024 & 204 & 24 & sam & $5 / 22 / 20$ & $5 / 27 / 2003$ & & 00 & 0.000 & 0.000 & 00 \\
\hline R04-024 & CR04 & 24 & duplicate & $5 / 22 / 2003$ & $6 / 4 / 2003$ & 0.006 & & 0.000 & 0.000 & \\
\hline A14-CR04-025 & CR04 & 25 & sample & $5 / 22 / 2003$ & $5 / 27 / 2003$ & 0.012 & 0.000 & 0.000 & 0.000 & 0.000 \\
\hline A14-CR04-025 & CR04 & 25 & duplicate & $5 / 22 / 2003$ & $6 / 4 / 2003$ & 0.004 & 0.000 & 0.000 & 0.000 & 0.000 \\
\hline A14-CR04-027 & CR04 & 27 & sam & $5 / 22 / 2003$ & $5 / 27 / 2003$ & .008 & 0.000 & 0.000 & 0.000 & 0.000 \\
\hline 4-027 & CR04 & 27 & dupli & $5 / 22 / 20$ & $6 / 4 / 2003$ & & & 000 & 0.000 & 0.000 \\
\hline R04-028 & CR04 & 28 & sam & $5 / 22 / 20$ & $5 / 27 / 2003$ & 0.000 & 0.000 & 0.000 & 0.000 & 0.000 \\
\hline A14-CR04-028 & CR04 & 28 & duplic & $5 / 22 / 2003$ & $6 / 4 / 2003$ & 0.0 & 0.000 & 0.000 & 0.000 & 0.000 \\
\hline A14-CR04-029 & CR04 & 29 & sam & $5 / 22 / 2003$ & $5 / 27 / 2003$ & 0.0 & 0.000 & 0.000 & 0.000 & 0.000 \\
\hline R04-029 & 204 & 29 & duplic & $5 / 22 / 2003$ & $6 / 4 / 2003$ & & 0.000 & 0.000 & 0.000 & 0.000 \\
\hline A14-CR04-030 & CR04 & 30 & cam & $5 / 22 / 2003$ & $5 / 27 / 2003$ & 0.000 & 0.000 & 0.000 & 0.000 & 0.000 \\
\hline A14-CR04-030 & CR04 & 30 & duplicate & $5 / 22 / 20$ & $6 / 4 / 2003$ & & & & 0.000 & 0.000 \\
\hline A14-CR04-031 & CR04 & 31 & sam & $5 / 22 / 2003$ & $5 / 27 / 2003$ & 0.000 & 0.000 & 0.000 & 0.000 & 0.000 \\
\hline A14-CR04-031 & CR04 & 31 & dupli & $5 / 22 / 2003$ & $6 / 4 / 2003$ & 0.000 & 0.000 & 0.000 & 0.000 & 0.000 \\
\hline A14-CR04-033 & CR04 & 33 & & $5 / 22 / 2003$ & $5 / 27 / 2003$ & & 0.000 & 0.000 & 0.000 & 0.000 \\
\hline A14-CR04-033 & & 33 & duplicate & $5 / 22 / 2003$ & $6 / 4 / 2003$ & & & 0.000 & 0.000 & 0.000 \\
\hline A14-CR04-035 & CR04 & 35 & sample & $5 / 22 / 2003$ & $5 / 27 / 2003$ & 0.000 & 0.000 & 0.000 & 0.000 & 0.000 \\
\hline
\end{tabular}




\begin{tabular}{|c|c|c|c|c|c|c|c|c|c|c|}
\hline Sample ID & Boring & Depth (ft) & Type & $\begin{array}{c}\text { Date } \\
\text { Collected }\end{array}$ & $\begin{array}{c}\text { Date } \\
\text { Analyzed }\end{array}$ & $\begin{array}{c}\text { PCE } \\
(\mathrm{mg} / \mathrm{kg})\end{array}$ & $\begin{array}{c}\text { TCE } \\
(\mathrm{mg} / \mathrm{kg})\end{array}$ & $\begin{array}{l}\text { C-DCE } \\
(\mathrm{mg} / \mathrm{kg})\end{array}$ & $\begin{array}{c}\text { TCA } \\
(\mathrm{mg} / \mathrm{kg})\end{array}$ & $\begin{array}{l}\text { 11DCE } \\
(\mathrm{mg} / \mathrm{kg})\end{array}$ \\
\hline A14-CR04-035 & CR04 & 35 & duplicate & $5 / 22 / 2003$ & $6 / 4 / 2003$ & 0.000 & 0.000 & 0.000 & 0.000 & 0.000 \\
\hline A14-CR04-037 & $\overline{\mathrm{R} 04}$ & 37 & sample & $5 / 22 / 2003$ & $5 / 27 / 2003$ & 000 & 000 & 000 & .000 & .000 \\
\hline A14-CR04-037 & CR04 & 37 & duplicate & $5 / 22 / 2003$ & 6/4/2003 & 0.000 & 0.000 & 0.000 & 0.000 & 0.000 \\
\hline A14-CR04-038 & CR04 & 38 & sample & $5 / 22 / 2003$ & $5 / 27 / 2003$ & 0.000 & 0.000 & 0.000 & 0.000 & 0.000 \\
\hline 14-CR04-038 & $\overline{\mathrm{R} 04}$ & 38 & duplicate & $5 / 22 / 2003$ & $6 / 4 / 2003$ & 0.000 & 00 & .000 & 0.000 & \\
\hline R04-039 & 204 & 39 & sample & $5 / 22 / 2003$ & $5 / 27 / 2003$ & 03 & 00 & 000 & 0.000 & .000 \\
\hline R04-039 & R04 & 39 & duplicate & $5 / 22 / 2003$ & 6/4/2003 & & & 00 & 0.000 & .000 \\
\hline A14-CR04-041 & CR04 & 41 & sample & $5 / 23 / 2003$ & $5 / 30 / 2003$ & 0.005 & 0.000 & 0.000 & 0.000 & 0.000 \\
\hline A14-CR04-041 & CR04 & 41 & duplicate & $5 / 23 / 2003$ & $6 / 5 / 2003$ & 0.000 & 0.000 & 0.000 & 0.000 & 0.000 \\
\hline R04-042 & R04 & 42 & le & $5 / 23 / 2003$ & $5 / 30 / 2003$ & 06 & .000 & 0.000 & 0.000 & 0.000 \\
\hline R04-042 & 204 & 42 & duplicate & $5 / 23 / 20$ & $6 / 5 / 2003$ & & & 00 & 0.000 & \\
\hline A14-CR04-043 & CR04 & 43 & sample & $5 / 23 / 2003$ & $5 / 30 / 2003$ & & 00 & 0.000 & 0.000 & 0.000 \\
\hline A14-CR04-043 & CR04 & 43 & duplicate & $5 / 23 / 2003$ & $6 / 5 / 2003$ & 0.000 & 0.000 & 0.000 & 0.000 & 0.000 \\
\hline 14-CR04-044 & CR04 & 44 & sample & $5 / 23 / 2003$ & $5 / 30 / 2003$ & 0.004 & 0.000 & 0.000 & 0.000 & 0.000 \\
\hline $4-044$ & 204 & 44 & duplic & $5 / 23 / 2$ & $6 / 5 / 2003$ & & 00 & 0.000 & 0.000 & 00 \\
\hline R04-045 & CR04 & 45 & sample & $5 / 23 / 20$ & $5 / 30 / 2003$ & & & 0.000 & 0.000 & \\
\hline A14-CR04-045 & CR04 & 45 & duplicate & $5 / 23 / 2003$ & 6/5/2003 & 0.000 & 0.000 & 0.000 & 0.000 & 0.000 \\
\hline A14-CR04-046 & CR04 & 46 & sample & $5 / 23 / 2003$ & $5 / 30 / 2003$ & 04 & 0.000 & 0.000 & 0.000 & 0.000 \\
\hline A14-CR04-046 & CR04 & 46 & duplicate & $5 / 23 / 2003$ & $6 / 5 / 2003$ & 0.000 & 0.000 & 0.000 & 0.000 & 0.000 \\
\hline R04-047 & CR04 & 47 & sample & $5 / 23 / 20$ & $5 / 30 / 2003$ & & & 0.000 & 0.000 & 0.000 \\
\hline R04-047 & CR04 & 47 & duplicate & $5 / 23 / 20$ & $6 / 5 / 2003$ & 00 & 0.000 & 0.000 & 0.000 & 0.000 \\
\hline A14-CR04-049 & CR04 & 49 & sample & $5 / 23 / 2003$ & $5 / 30 / 2003$ & & 0.000 & 0.000 & 0.000 & 0.000 \\
\hline A14-CR04-049 & CR04 & 49 & duplicate & $5 / 23 / 2003$ & $6 / 5 / 2003$ & 0.0 & 0.000 & 0.000 & 0.000 & 0.000 \\
\hline 14-CR04-050 & CR04 & 50 & sample & $5 / 23 / 2003$ & $5 / 30 / 2003$ & 00 & 00 & 0.000 & 0.000 & 0.000 \\
\hline A14-CR04-050 & CR04 & 50 & duplicate & $5 / 23 / 2003$ & $6 / 5 / 2003$ & .000 & .000 & 0.000 & 0.000 & 0.000 \\
\hline A14-CR04-052 & CR04 & 52 & sample & $5 / 23 / 20$ & $5 / 30 / 2003$ & & & & 0.000 & 0.000 \\
\hline A14-CR04-052 & CR04 & 52 & duplicate & $5 / 23 / 2003$ & $6 / 5 / 2003$ & 0.000 & 0.000 & 0.000 & 0.000 & 0.000 \\
\hline A14-CR04-053 & CR04 & 53 & & $5 / 23 / 2003$ & $5 / 30 / 2003$ & 0.006 & 0.000 & 0.000 & 0.000 & 0.000 \\
\hline A14-CR04-053 & CR04 & 53 & duplicate & $5 / 23 / 2003$ & $6 / 5 / 2003$ & & 0.000 & 0.000 & 0.000 & 0.000 \\
\hline A14-CR04-054 & & 54 & & $5 / 23 / 2003$ & $5 / 30 / 2003$ & & & 0.000 & 0.000 & 0.000 \\
\hline A14-CR04-054 & CR04 & 54 & duplicate & $5 / 23 / 2003$ & $6 / 5 / 2003$ & 0.000 & 0.000 & 0.000 & 0.000 & 0.000 \\
\hline
\end{tabular}




\begin{tabular}{|c|c|c|c|c|c|c|c|c|c|c|}
\hline Sample ID & Boring & Depth (ft) & Type & $\begin{array}{c}\text { Date } \\
\text { Collected }\end{array}$ & $\begin{array}{c}\text { Date } \\
\text { Analyzed }\end{array}$ & $\begin{array}{c}\text { PCE } \\
(\mathrm{mg} / \mathrm{kg})\end{array}$ & $\begin{array}{c}\text { TCE } \\
(\mathrm{mg} / \mathrm{kg})\end{array}$ & $\begin{array}{l}\text { C-DCE } \\
(\mathrm{mg} / \mathrm{kg})\end{array}$ & $\begin{array}{c}\text { TCA } \\
(\mathrm{mg} / \mathrm{kg})\end{array}$ & $\begin{array}{l}\text { 11DCE } \\
(\mathrm{mg} / \mathrm{kg})\end{array}$ \\
\hline A14-CR04-055 & CR04 & 55 & sample & $5 / 23 / 2003$ & $5 / 30 / 2003$ & 0.004 & 0.000 & 0.000 & 0.000 & 0.000 \\
\hline A14-CR04-055 & $\overline{R 04}$ & 55 & duplicate & $5 / 23 / 2003$ & $6 / 5 / 2003$ & .000 & .000 & 000 & .000 & .000 \\
\hline A14-CR04-056 & CR04 & 56 & sample & //23/2003 & $5 / 30 / 2003$ & 0.007 & 0.000 & 0.000 & 0.000 & 0.000 \\
\hline A14-CR04-056 & CR04 & 56 & duplicate & $5 / 23 / 2003$ & $6 / 5 / 2003$ & 0.000 & 0.000 & 0.000 & 0.000 & 0.000 \\
\hline 14-CR04-057 & R04 & 57 & sample & $5 / 23 / 2003$ & $5 / 30 / 2003$ & 0.004 & 000 & .000 & 0.000 & \\
\hline R04-057 & 204 & 57 & duplicate & $5 / 23 / 2003$ & $6 / 5 / 2003$ & 000 & 00 & 00 & .000 & .000 \\
\hline R04-058 & 204 & 58 & sample & $5 / 23 / 2003$ & $5 / 30 / 2003$ & 04 & 00 & 000 & 0.000 & .000 \\
\hline A14-CR04-058 & CR04 & 58 & duplicate & $5 / 23 / 2003$ & $6 / 5 / 2003$ & 0.000 & 0.000 & 0.000 & 0.000 & 0.000 \\
\hline A14-CR04-059 & CR04 & 59 & sam & $5 / 23 / 2003$ & $5 / 30 / 2003$ & 0.003 & 0.000 & 0.000 & 0.000 & 0.000 \\
\hline 14-CR04-059 & R04 & 59 & dupli & $5 / 23 / 20$ & $6 / 5 / 2003$ & 00 & 00 & 0.000 & 0.000 & \\
\hline 44-060 & 204 & 60 & sam & $5 / 23 / 20$ & $5 / 30 / 2003$ & & & 00 & 0.000 & \\
\hline A14-CR04-060 & CR04 & 60 & duplicate & $5 / 23 / 20$ & $6 / 5 / 2003$ & 000 & 000 & 0.000 & 0.000 & 0.000 \\
\hline A14-CR04-061 & CR04 & 61 & sample & $5 / 23 / 2003$ & $5 / 30 / 2003$ & 0.005 & 0.000 & 0.000 & 0.000 & 0.000 \\
\hline 14-CR04-061 & CR04 & 61 & duplicate & $5 / 23 / 2003$ & $6 / 5 / 2003$ & 0.000 & 0.000 & 0.000 & 0.000 & 0.000 \\
\hline R04-062 & 204 & 62 & sam & $5 / 23 / 2$ & $5 / 30 / 2003$ & & & 0.000 & 0.000 & 0.000 \\
\hline R04-062 & CR04 & 62 & duplicate & $5 / 23 / 20$ & $6 / 5 / 2003$ & 0.000 & & 0.000 & 0.000 & \\
\hline A14-CR04-063 & CR04 & 63 & sample & $5 / 23 / 2003$ & $5 / 30 / 2003$ & 0.004 & 0.000 & 0.000 & 0.000 & 0.000 \\
\hline A14-CR04-063 & CR04 & 63 & duplicate & $5 / 23 / 2003$ & $6 / 5 / 2003$ & 0.000 & 0.000 & 0.000 & 0.000 & 0.000 \\
\hline A14-CR04-064 & CR04 & 64 & sam & $5 / 23 / 2003$ & $5 / 30 / 2003$ & .003 & 0.000 & 0.000 & 0.000 & 0.000 \\
\hline 04-064 & CR04 & 64 & dupli & $5 / 23 / 20$ & $6 / 5 / 2003$ & & & 0.000 & 0.000 & 0.000 \\
\hline A14-CR04-065 & CR04 & 65 & sam & $5 / 23 / 20$ & $5 / 30 / 2003$ & 0.007 & 0.000 & 0.000 & 0.000 & 0.000 \\
\hline A14-CR04-065 & CR04 & 65 & duplic & $5 / 23 / 2003$ & $6 / 5 / 2003$ & 0.0 & 0.000 & 0.000 & 0.000 & 0.000 \\
\hline A14-CR04-067 & CR04 & 67 & sam & $5 / 23 / 2003$ & $5 / 30 / 2003$ & 0.0 & 0.000 & 0.000 & 0.000 & 0.000 \\
\hline 14-CR04-067 & 204 & 67 & duplic & $5 / 23 / 2003$ & $6 / 5 / 2003$ & 0.0 & 0.000 & 0.000 & 0.000 & 0.000 \\
\hline A14-CR04-069 & CR04 & 69 & cam & $5 / 23 / 2003$ & $5 / 30 / 2003$ & 0.000 & 0.000 & 0.000 & 0.000 & 0.000 \\
\hline A14-CR04-069 & CR04 & 69 & duplicate & $5 / 23 / 20$ & $6 / 5 / 2003$ & & & & 0.000 & 0.000 \\
\hline A14-CR04-071 & CR04 & 71 & sample & $5 / 23 / 2003$ & $5 / 30 / 2003$ & 0.003 & 0.000 & 0.000 & 0.000 & 0.000 \\
\hline A14-CR04-071 & CR04 & 71 & dupli & $5 / 23 / 2003$ & $6 / 5 / 2003$ & 0.000 & 0.000 & 0.000 & 0.000 & 0.000 \\
\hline A14-CR04-073 & CR04 & 73 & & $5 / 23 / 2003$ & $5 / 30 / 2003$ & 0.006 & 0.000 & 0.000 & 0.000 & 0.000 \\
\hline A14-CR04-073 & & 73 & duplicate & $5 / 23 / 2003$ & $6 / 5 / 2003$ & & & 0.000 & 0.000 & 0.000 \\
\hline A14-CR04-075 & CR04 & 75 & sample & $5 / 23 / 2003$ & $5 / 30 / 2003$ & 0.005 & 0.000 & 0.000 & 0.000 & 0.000 \\
\hline
\end{tabular}




\begin{tabular}{|c|c|c|c|c|c|c|c|c|c|c|}
\hline Sample ID & Boring & Depth (ft) & Type & $\begin{array}{c}\text { Date } \\
\text { Collected }\end{array}$ & $\begin{array}{c}\text { Date } \\
\text { Analyzed }\end{array}$ & $\begin{array}{c}\text { PCE } \\
(\mathrm{mg} / \mathrm{kg})\end{array}$ & $\begin{array}{c}\text { TCE } \\
(\mathrm{mg} / \mathrm{kg})\end{array}$ & $\begin{array}{l}\text { C-DCE } \\
(\mathrm{mg} / \mathrm{kg})\end{array}$ & $\begin{array}{c}\text { TCA } \\
(\mathrm{mg} / \mathrm{kg})\end{array}$ & $\begin{array}{l}\text { 11DCE } \\
(\mathrm{mg} / \mathrm{kg})\end{array}$ \\
\hline A14-CR04-075 & CR04 & 75 & duplicate & $5 / 23 / 2003$ & $6 / 5 / 2003$ & 0.000 & 0.000 & 0.000 & 0.000 & 0.000 \\
\hline A14-CR04-077 & $\overline{\mathrm{R} 04}$ & 77 & sample & /23/2003 & $5 / 30 / 2003$ & 004 & 000 & 000 & .000 & .000 \\
\hline A14-CR04-077 & CR04 & 77 & duplicate & //23/2003 & 6/5/2003 & 0.000 & 0.000 & 0.000 & 0.000 & 0.000 \\
\hline A14-CR04-079 & CR04 & 79 & sample & /23/2003 & $5 / 30 / 2003$ & 0.004 & 0.000 & 0.000 & 0.000 & 0.000 \\
\hline 14-CR04-079 & $\overline{R 04}$ & 79 & duplicate & $5 / 23 / 2003$ & $6 / 5 / 2003$ & .000 & 00 & .000 & 0.000 & \\
\hline R04-081 & 204 & 81 & sample & $5 / 23 / 2003$ & $5 / 30 / 2003$ & 03 & & 00 & .000 & .000 \\
\hline R04-081 & R04 & 81 & duplicate & $5 / 23 / 2003$ & $6 / 5 / 2003$ & & & 00 & 0.000 & .000 \\
\hline A14-CR04-082 & CR04 & 82 & sample & $5 / 23 / 2003$ & $5 / 30 / 2003$ & 0.004 & 0.000 & 0.000 & 0.000 & 0.000 \\
\hline A14-CR04-082 & CR04 & 82 & duplicate & $5 / 23 / 2003$ & $6 / 5 / 2003$ & 0.000 & 0.000 & 0.000 & 0.000 & 0.000 \\
\hline 14-CR04-083 & R04 & 83 & ole & $5 / 23 / 20$ & $5 / 30 / 2003$ & & & 0.000 & 0.000 & \\
\hline $4-083$ & 204 & 83 & duplicate & $5 / 23 / 20$ & $6 / 5 / 2003$ & & & 00 & 000 & \\
\hline A14-CR04-085 & CR04 & 85 & sample & $5 / 23 / 2003$ & $5 / 30 / 2003$ & & & .000 & 0.000 & 0.000 \\
\hline A14-CR04-085 & CR04 & 85 & duplicate & $5 / 23 / 2003$ & $6 / 5 / 2003$ & 0.000 & 0.000 & 0.000 & 0.000 & 0.000 \\
\hline 14-CR04-086 & CR04 & 86 & sample & $5 / 23 / 2003$ & $5 / 30 / 2003$ & 0.003 & 0.000 & 0.000 & 0.000 & 0.000 \\
\hline R04-086 & 204 & 86 & duplic & $5 / 23 / 2$ & $6 / 5 / 2003$ & & & 0.000 & 0.000 & 00 \\
\hline R04-087 & CR04 & 87 & sample & $5 / 23 / 20$ & $5 / 30 / 2003$ & & & 0.000 & 0.000 & \\
\hline A14-CR04-087 & CR04 & 87 & duplicate & $5 / 23 / 2003$ & $6 / 5 / 2003$ & 0.002 & 0.000 & 0.000 & 0.000 & 0.000 \\
\hline A14-CR04-088 & CR04 & 88 & sample & $5 / 23 / 2003$ & $5 / 30 / 2003$ & 05 & 0.000 & 0.000 & 0.000 & 0.000 \\
\hline A14-CR04-088 & CR04 & 88 & duplicate & $5 / 23 / 2003$ & $6 / 5 / 2003$ & .000 & 0.000 & 0.000 & 0.000 & 0.000 \\
\hline 04-089 & CR04 & 89 & sample & $5 / 23 / 20$ & $5 / 30 / 2003$ & & & 000 & 0.000 & 0.000 \\
\hline R04-089 & CR04 & 89 & duplicate & $5 / 23 / 20$ & $6 / 5 / 2003$ & 00 & 0.000 & 0.000 & 0.000 & 0.000 \\
\hline A14-CR04-100 & CR04 & 100 & sample & $5 / 23 / 2003$ & $5 / 30 / 2003$ & & & 0.000 & 0.000 & 0.000 \\
\hline A14-CR04-100 & CR04 & 100 & duplicate & $5 / 23 / 2003$ & $6 / 5 / 2003$ & 0.0 & 0.000 & 0.000 & 0.000 & 0.000 \\
\hline A14-CR04-101 & 804 & 101 & sample & $5 / 23 / 2003$ & $5 / 30 / 2003$ & & 0.000 & 0.000 & 0.000 & 0.000 \\
\hline A14-CR04-101 & CR04 & 101 & duplicate & $5 / 23 / 2003$ & $6 / 5 / 2003$ & .000 & .000 & 0.000 & 0.000 & 0.000 \\
\hline A14-CR04-105 & CR04 & & sample & $5 / 23 / 20$ & $5 / 30 / 2003$ & & & & 0.000 & 0.000 \\
\hline A14-CR04-105 & CR04 & 105 & duplicate & $5 / 23 / 2003$ & $6 / 5 / 2003$ & 0.000 & 0.000 & 0.000 & 0.000 & 0.000 \\
\hline A14-CR04-106 & CR04 & 106 & & $5 / 23 / 2003$ & $5 / 30 / 2003$ & 0.005 & 0.000 & 0.000 & 0.000 & 0.000 \\
\hline A14-CR04-106 & CR04 & 106 & duplicate & $5 / 23 / 2003$ & $6 / 5 / 2003$ & & 0.000 & 0.000 & 0.000 & 0.000 \\
\hline A14-CR04-110 & & 110 & & $5 / 23 / 2003$ & $5 / 30 / 2003$ & & & 0.000 & 0.000 & 0.000 \\
\hline A14-CR04-110 & CR04 & 110 & duplicate & $5 / 23 / 2003$ & $6 / 5 / 2003$ & 0.007 & 0.000 & 0.000 & 0.000 & 0.000 \\
\hline
\end{tabular}




\begin{tabular}{|c|c|c|c|c|c|c|c|c|c|c|}
\hline Sample ID & Boring & Depth $(\mathrm{ft})$ & Type & $\begin{array}{c}\text { Date } \\
\text { Collected }\end{array}$ & $\begin{array}{c}\text { Date } \\
\text { Analyzed }\end{array}$ & $\begin{array}{c}\text { PCE } \\
(\mathrm{mg} / \mathrm{kg})\end{array}$ & $\begin{array}{c}\text { TCE } \\
(\mathrm{mg} / \mathrm{kg})\end{array}$ & $\begin{array}{c}\text { C-DCE } \\
(\mathrm{mg} / \mathrm{kg})\end{array}$ & $\begin{array}{c}\text { TCA } \\
(\mathrm{mg} / \mathrm{kg})\end{array}$ & $\begin{array}{c}11 \mathrm{DCE} \\
(\mathrm{mg} / \mathrm{kg})\end{array}$ \\
\hline A14-CR04-111 & CR04 & 111 & sample & $5 / 23 / 2003$ & $5 / 30 / 2003$ & 0.021 & 0.000 & 0.000 & 0.000 & 0.000 \\
\hline A14-CR04-111 & CR04 & 111 & duplicate & $5 / 23 / 2003$ & $6 / 5 / 2003$ & 0.019 & 0.000 & 0.000 & 0.000 & 0.000 \\
\hline A14-CR04-112 & CR04 & 112 & sample & $5 / 23 / 2003$ & $5 / 30 / 2003$ & 0.146 & 0.022 & 0.000 & 0.000 & 0.000 \\
\hline A14-CR04-112 & CR04 & 112 & duplicate & $5 / 23 / 2003$ & $6 / 5 / 2003$ & 0.142 & 0.024 & 0.000 & 0.000 & 0.000 \\
\hline A14-CR04-117 & CR04 & 117 & sample & $5 / 23 / 2003$ & $5 / 30 / 2003$ & 0.014 & 0.006 & 0.000 & 0.000 & 0.000 \\
\hline A14-CR04-117 & CR04 & 117 & duplicate & $5 / 23 / 2003$ & $6 / 5 / 2003$ & 0.017 & 0.009 & 0.000 & 0.000 & 0.000 \\
\hline A14-CR04-118 & CR04 & 118 & sample & $5 / 23 / 2003$ & $5 / 30 / 2003$ & 0.056 & 0.027 & 0.000 & 0.000 & 0.000 \\
\hline A14-CR04-118 & CR04 & 118 & duplicate & $5 / 23 / 2003$ & $6 / 5 / 2003$ & 0.054 & 0.026 & 0.000 & 0.000 & 0.000 \\
\hline
\end{tabular}


Table 10 - Sediment Analysis Results for Boring CR05

\begin{tabular}{|c|c|c|c|c|c|c|c|c|c|c|}
\hline Sample ID & Boring & Depth (ft) & Type & $\begin{array}{c}\text { Date } \\
\text { Collected }\end{array}$ & $\begin{array}{c}\text { Date } \\
\text { Analyzed }\end{array}$ & $\begin{array}{c}\text { PCE } \\
(\mathrm{mg} / \mathrm{kg})\end{array}$ & $\begin{array}{c}\text { TCE } \\
(\mathrm{mg} / \mathrm{kg})\end{array}$ & $\begin{array}{l}\text { C-DCE } \\
(\mathrm{mg} / \mathrm{kg})\end{array}$ & $\begin{array}{c}\text { TCA } \\
(\mathrm{mg} / \mathrm{kg})\end{array}$ & $\begin{array}{l}11 \mathrm{DCE} \\
(\mathrm{mg} / \mathrm{kg})\end{array}$ \\
\hline A14-CR05-01 & CR05 & 1 & sample & $5 / 27 / 2003$ & $5 / 29 / 2003$ & 0.000 & 0.000 & 0.000 & 0.000 & 0.000 \\
\hline A14-CR05-01 & CR05 & 1 & duplicate & $5 / 27 / 2003$ & $6 / 5 / 03$ & 0.000 & 0.000 & 0.000 & 0.000 & 0.000 \\
\hline A14-CR05-02 & CR05 & 2 & sample & $5 / 27 / 2003$ & $5 / 29 / 2003$ & 0.000 & 0.000 & 0.000 & 0.000 & 0.000 \\
\hline A14-CR05-02 & CR05 & 2 & duplicate & $5 / 27 / 2003$ & $6 / 5 / 03$ & 0.006 & 0.000 & 0.000 & 0.000 & 0.000 \\
\hline A14-CR05-03 & CR05 & 3 & sample & $5 / 27 / 2003$ & $5 / 29 / 2003$ & 0.255 & 0.014 & 0.000 & 0.000 & 0.000 \\
\hline A14-CR05-03 & CR05 & 3 & duplicate & $5 / 27 / 2003$ & $6 / 5 / 03$ & 0.395 & 0.013 & 0.000 & 0.000 & 0.000 \\
\hline A14-CR05-04 & CR05 & 4 & sample & $5 / 27 / 2003$ & $5 / 29 / 2003$ & 2.088 & 0.100 & 0.198 & 0.000 & 0.000 \\
\hline A14-CR05-04 & CR05 & 4 & duplicate & $5 / 27 / 2003$ & $6 / 5 / 03$ & 1.771 & 0.051 & 0.111 & 0.000 & 0.000 \\
\hline A14-CR05-06 & CR05 & 6 & sample & $5 / 27 / 2003$ & $5 / 29 / 2003$ & 0.198 & 0.013 & 0.000 & 0.000 & 0.000 \\
\hline A14-CR05-06 & CR05 & 6 & duplicate & $5 / 27 / 2003$ & $6 / 5 / 03$ & 0.321 & 0.011 & 0.000 & 0.000 & 0.000 \\
\hline A14-CR05-07 & CR05 & 7 & sample & $5 / 27 / 2003$ & $5 / 29 / 2003$ & 0.566 & 0.051 & 0.183 & 0.000 & 0.000 \\
\hline A14-CR05-07 & CR05 & 7 & duplicate & $5 / 27 / 2003$ & $6 / 5 / 03$ & 1.067 & 0.061 & 0.181 & 0.000 & 0.000 \\
\hline A14-CR05-08 & CR05 & 8 & sample & $5 / 27 / 2003$ & $5 / 29 / 2003$ & 0.527 & 0.051 & 0.187 & 0.000 & 0.000 \\
\hline A14-CR05-08 & CR05 & 8 & duplicate & $5 / 27 / 2003$ & $6 / 5 / 03$ & 0.503 & 0.027 & 0.123 & 0.000 & 0.000 \\
\hline A14-CR05-09 & CR05 & 9 & sample & $5 / 27 / 2003$ & $5 / 29 / 2003$ & 0.654 & 0.059 & 0.213 & 0.000 & 0.000 \\
\hline A14-CR05-09 & CR05 & 9 & duplicate & $5 / 27 / 2003$ & $6 / 5 / 03$ & 0.336 & 0.018 & 0.089 & 0.000 & 0.000 \\
\hline A14-CR05-10 & CR05 & 10 & sample & $5 / 27 / 2003$ & $5 / 29 / 2003$ & 0.487 & 0.050 & 0.196 & 0.000 & 0.000 \\
\hline A14-CR05-10 & CR05 & 10 & duplicate & $5 / 27 / 2003$ & $6 / 5 / 03$ & 1.042 & 0.053 & 0.179 & 0.000 & 0.000 \\
\hline A14-CR05-11 & CR05 & 11 & sample & $5 / 27 / 2003$ & $5 / 29 / 2003$ & 1.108 & 0.083 & 0.301 & 0.000 & 0.000 \\
\hline A14-CR05-11 & CR05 & 11 & \begin{tabular}{|l} 
duplicate \\
\end{tabular} & $5 / 27 / 2003$ & $6 / 5 / 03$ & 0.583 & 0.026 & 0.161 & 0.000 & 0.000 \\
\hline A14-CR05-12 & CR05 & 12 & sample & $5 / 27 / 2003$ & $5 / 29 / 2003$ & 0.417 & 0.036 & 0.141 & 0.000 & 0.000 \\
\hline A14-CR05-12 & CR05 & 12 & duplicate & $5 / 27 / 2003$ & $6 / 5 / 03$ & 0.624 & 0.031 & 0.131 & 0.000 & 0.000 \\
\hline A14-CR05-13 & CR05 & 13 & sample & $5 / 27 / 2003$ & $5 / 29 / 2003$ & 0.273 & 0.025 & 0.113 & 0.000 & 0.000 \\
\hline A14-CR05-13 & CR05 & 13 & duplicate & $5 / 27 / 2003$ & $6 / 5 / 03$ & 0.275 & 0.014 & 0.081 & 0.000 & 0.000 \\
\hline A14-CR05-14 & CR05 & 14 & sample & $5 / 27 / 2003$ & $5 / 29 / 2003$ & 0.491 & 0.051 & 0.154 & 0.000 & 0.000 \\
\hline A14-CR05-14 & CR05 & 14 & duplicate & $5 / 27 / 2003$ & $6 / 5 / 03$ & 0.259 & 0.015 & 0.000 & 0.000 & 0.000 \\
\hline A14-CR05-15 & CR05 & 15 & sample & $5 / 27 / 2003$ & $5 / 29 / 2003$ & 0.139 & 0.010 & 0.000 & 0.000 & 0.000 \\
\hline A14-CR05-15 & CR05 & 15 & duplicate & $5 / 27 / 2003$ & $6 / 5 / 03$ & 0.348 & 0.020 & 0.080 & 0.000 & 0.000 \\
\hline
\end{tabular}




\begin{tabular}{|c|c|c|c|c|c|c|c|c|c|c|}
\hline Sample ID & Boring & Depth (ft) & Type & $\begin{array}{c}\text { Date } \\
\text { Collected }\end{array}$ & $\begin{array}{c}\text { Date } \\
\text { Analyzed }\end{array}$ & $\begin{array}{c}\text { PCE } \\
(\mathrm{mg} / \mathrm{kg})\end{array}$ & $\begin{array}{c}\text { TCE } \\
(\mathrm{mg} / \mathrm{kg})\end{array}$ & $\begin{array}{l}\text { C-DCE } \\
(\mathrm{mg} / \mathrm{kg})\end{array}$ & $\begin{array}{c}\text { TCA } \\
(\mathrm{mg} / \mathrm{kg})\end{array}$ & $\begin{array}{l}\text { 11DCE } \\
\text { (mg/kg) }\end{array}$ \\
\hline A14-CR05-16 & CR05 & 16 & sample & $5 / 27 / 2003$ & $5 / 29 / 2003$ & 0.143 & 0.015 & 0.049 & 0.000 & 0.000 \\
\hline A14-CR05-16 & CR05 & 16 & duplicate & $5 / 27 / 2003$ & $6 / 5 / 03$ & 0.212 & 0.012 & 0.057 & 0.000 & 0.000 \\
\hline A14-CR05-17 & CR05 & 17 & sample & $5 / 27 / 2003$ & $5 / 29 / 2003$ & 0.140 & 0.013 & 0.053 & 0.000 & 0.000 \\
\hline A14-CR05-17 & CR05 & 17 & duplicate & $5 / 27 / 2003$ & $6 / 5 / 03$ & 0.115 & 0.010 & 0.041 & 0.000 & 0.000 \\
\hline A14-CR05-18 & CR05 & 18 & sample & $5 / 27 / 2003$ & $5 / 29 / 2003$ & 0.292 & 0.028 & 0.144 & 0.000 & 0.000 \\
\hline A14-CR05-18 & CR05 & 18 & duplicate & $5 / 27 / 2003$ & $6 / 5 / 03$ & 0.076 & 0.006 & 0.000 & 0.000 & 0.000 \\
\hline A14-CR05-19 & CR05 & 19 & sample & $5 / 27 / 2003$ & $5 / 29 / 2003$ & 0.472 & 0.007 & 0.000 & 0.000 & 0.000 \\
\hline A14-CR05-19 & CR05 & 19 & duplicate & $5 / 27 / 2003$ & $6 / 5 / 03$ & 0.564 & 0.005 & 0.000 & 0.000 & 0.000 \\
\hline A14-CR05-20 & CR05 & 20 & sample & $5 / 27 / 2003$ & $5 / 29 / 2003$ & 0.686 & 0.006 & 0.000 & 0.000 & 0.000 \\
\hline A14-CR05-20 & CR05 & 20 & duplicate & $5 / 27 / 2003$ & $6 / 5 / 03$ & 0.911 & 0.004 & 0.000 & 0.000 & 0.000 \\
\hline A14-C & CR05 & 21 & sample & $5 / 27 / 2003$ & $5 / 29 / 2003$ & 0.004 & 0.000 & 0.000 & 0.000 & 0.000 \\
\hline A14-CR05-21 & CR05 & 21 & duplicate & $5 / 27 / 2003$ & $6 / 5 / 03$ & 0.021 & 0.005 & 0.000 & 0.000 & 0.000 \\
\hline A14-CR05-22 & CR05 & 22 & sample & $5 / 27 / 2003$ & $5 / 29 / 2003$ & 0.000 & 0.000 & 0.000 & 0.000 & 0.000 \\
\hline A14-CR05-22 & CR05 & 22 & duplicate & $5 / 27 / 2003$ & $6 / 5 / 03$ & 0.000 & 0.000 & 0.000 & 0.000 & 0.000 \\
\hline A14-CR05-23 & CR05 & 23 & sample & $5 / 27 / 2003$ & $5 / 29 / 2003$ & 0.008 & 0.000 & 0.000 & 0.000 & 0.000 \\
\hline $\mathrm{A} 14-\mathrm{C}$ & CR05 & 23 & duplicate & $5 / 27 / 2003$ & $6 / 5 / 03$ & 0.004 & 0.000 & 0.000 & 0.000 & 0.000 \\
\hline A14-CR05-24 & CR05 & 24 & sample & $5 / 27 / 2003$ & $5 / 29 / 2003$ & 0.000 & 0.000 & 0.000 & 0.000 & 0.000 \\
\hline A14-CR05-24 & CR05 & 24 & duplicate & $5 / 27 / 2003$ & $6 / 5 / 03$ & 0.000 & 0.000 & 0.000 & 0.000 & 0.000 \\
\hline A14-CR05-25 & CR05 & 25 & sample & $5 / 27 / 2003$ & $5 / 29 / 2003$ & 0.000 & 0.000 & 0.000 & 0.000 & 0.000 \\
\hline A14-CR05-25 & CR05 & 25 & duplicate & $5 / 27 / 2003$ & $6 / 5 / 03$ & 0.000 & 0.000 & 0.000 & 0.000 & 0.000 \\
\hline A14-CR05-26 & CR05 & 26 & sample & $5 / 27 / 2003$ & $5 / 29 / 2003$ & 0.000 & 0.000 & 0.000 & 0.000 & 0.000 \\
\hline A14-CR05-26 & CR05 & 26 & duplicate & $5 / 27 / 2003$ & $6 / 5 / 03$ & 0.003 & 0.000 & 0.000 & 0.000 & 0.000 \\
\hline A14-CR05-27 & CR05 & 27 & sample & $5 / 27 / 2003$ & $5 / 29 / 2003$ & 0.000 & 0.000 & 0.000 & 0.000 & 0.000 \\
\hline A14-CR05-27 & CR05 & 27 & duplicate & $5 / 27 / 2003$ & $6 / 5 / 03$ & 0.000 & 0.000 & 0.000 & 0.000 & 0.000 \\
\hline A14-CR05-28 & CR05 & 28 & sample & $5 / 27 / 2003$ & $5 / 29 / 2003$ & 0.000 & 0.000 & 0.000 & 0.000 & 0.000 \\
\hline A14-CR05-28 & CR05 & 28 & duplicate & $5 / 27 / 2003$ & $6 / 5 / 03$ & 0.000 & 0.000 & 0.000 & 0.000 & 0.000 \\
\hline A14-CR05-29 & CR05 & 29 & sample & $5 / 27 / 2003$ & $5 / 29 / 2003$ & 0.000 & 0.000 & 0.000 & 0.000 & 0.000 \\
\hline A14-CR05-29 & CR05 & 29 & duplicate & $5 / 27 / 2003$ & $6 / 5 / 03$ & 0.000 & 0.000 & 0.000 & 0.000 & 0.000 \\
\hline A14-CR05-30 & CR05 & 30 & sample & $5 / 27 / 2003$ & $5 / 29 / 2003$ & 0.000 & 0.000 & 0.000 & 0.000 & 0.000 \\
\hline A14-CR05-30 & CR05 & 30 & duplicate & $5 / 27 / 2003$ & $6 / 5 / 03$ & 0.000 & 0.000 & 0.000 & 0.000 & 0.000 \\
\hline A14-CR05-31 & CR05 & 31 & sample & $5 / 27 / 2003$ & $5 / 29 / 2003$ & 0.000 & 0.000 & 0.000 & 0.000 & 0.000 \\
\hline
\end{tabular}




\begin{tabular}{|c|c|c|c|c|c|c|c|c|c|c|}
\hline Sample ID & Boring & Depth (ft) & Type & $\begin{array}{c}\text { Date } \\
\text { Collected }\end{array}$ & $\begin{array}{c}\text { Date } \\
\text { Analyzed }\end{array}$ & $\begin{array}{c}\text { PCE } \\
(\mathrm{mg} / \mathrm{kg})\end{array}$ & $\begin{array}{c}\text { TCE } \\
(\mathrm{mg} / \mathrm{kg})\end{array}$ & $\begin{array}{l}\text { C-DCE } \\
(\mathrm{mg} / \mathrm{kg})\end{array}$ & $\begin{array}{c}\text { TCA } \\
(\mathrm{mg} / \mathrm{kg})\end{array}$ & $\begin{array}{l}\text { 11DCE } \\
(\mathrm{mg} / \mathrm{kg})\end{array}$ \\
\hline A14-CR05-31 & CR05 & 31 & \begin{tabular}{|l} 
duplicate \\
\end{tabular} & $5 / 27 / 2003$ & $6 / 5 / 03$ & 0.000 & 0.000 & 0.000 & 0.000 & 0.000 \\
\hline A14-CR05-32 & CR05 & 32 & sample & $5 / 27 / 2003$ & $5 / 29 / 2003$ & 0.000 & 0.000 & 0.000 & 0.000 & 0.000 \\
\hline A14-CR05-32 & CR05 & 32 & duplicate & $5 / 27 / 2003$ & $6 / 5 / 03$ & 0.000 & 0.000 & 0.000 & 0.000 & 0.000 \\
\hline A14-CR05-33 & CR05 & 33 & sample & $5 / 27 / 2003$ & $5 / 29 / 2003$ & 0.000 & 0.000 & 0.000 & 0.000 & 0.000 \\
\hline A14-CR05-33 & CR05 & 33 & duplicate & $5 / 27 / 2003$ & $6 / 5 / 03$ & 0.000 & 0.000 & 0.000 & 0.000 & 0.000 \\
\hline A14-CR05-34 & CR05 & 34 & sample & $5 / 27 / 2003$ & $5 / 29 / 2003$ & 0.000 & 0.000 & 0.000 & 0.000 & 0.000 \\
\hline A14-CR05-34 & CR05 & 34 & duplicate & $5 / 27 / 2003$ & $6 / 5 / 03$ & 0.000 & 0.000 & 0.000 & 0.000 & 0.000 \\
\hline A14-CR05-35 & CR05 & 35 & sample & $5 / 27 / 2003$ & $5 / 29 / 2003$ & 0.000 & 0.000 & 0.000 & 0.000 & 0.000 \\
\hline A14-CR05-35 & CR05 & 35 & duplicate & $5 / 27 / 2003$ & $6 / 5 / 03$ & 0.000 & 0.000 & 0.000 & 0.000 & 0.000 \\
\hline A14-CR05-36 & CR05 & 36 & sample & $5 / 27 / 2003$ & $5 / 29 / 2003$ & 0.000 & 0.000 & 0.000 & 0.000 & 0.000 \\
\hline A14-C & CR05 & 36 & duplicate & $5 / 27 / 2003$ & $6 / 5 / 03$ & 0.000 & 0.000 & 0.000 & 0.000 & 0.000 \\
\hline A14-CR05-37 & CR05 & 37 & sample & $5 / 27 / 2003$ & $5 / 29 / 2003$ & 0.000 & 0.000 & 0.000 & 0.000 & 0.000 \\
\hline A14-CR05-37 & CR05 & 37 & duplicate & $5 / 27 / 2003$ & $6 / 5 / 03$ & 0.000 & 0.000 & 0.000 & 0.000 & 0.000 \\
\hline A14-CR05-38 & CR05 & 38 & sample & $5 / 27 / 2003$ & $5 / 29 / 2003$ & 0.000 & 0.000 & 0.000 & 0.000 & 0.000 \\
\hline A14-CR05-38 & CR05 & 38 & duplicate & $5 / 27 / 2003$ & $6 / 5 / 03$ & 0.000 & 0.000 & 0.000 & 0.000 & 0.000 \\
\hline $\mathrm{A} 14-\mathrm{C}$ & CR05 & 39 & sample & $5 / 27 / 2003$ & $5 / 29 / 2003$ & 0.000 & 0.000 & 0.000 & 0.000 & 0.000 \\
\hline A14-CR05-39 & CR05 & 39 & duplicate & $5 / 27 / 2003$ & $6 / 5 / 03$ & 0.000 & 0.000 & 0.000 & 0.000 & 0.000 \\
\hline A14-CR05-40 & CR05 & 40 & sample & $5 / 27 / 2003$ & $5 / 29 / 2003$ & 0.000 & 0.000 & 0.000 & 0.000 & 0.000 \\
\hline A14-CR05-40 & CR05 & 40 & duplicate & $5 / 27 / 2003$ & $6 / 5 / 03$ & 0.000 & 0.000 & 0.000 & 0.000 & 0.000 \\
\hline A14-CR05-41 & CR05 & 41 & sample & $5 / 27 / 2003$ & $5 / 29 / 2003$ & 0.000 & 0.000 & 0.000 & 0.000 & 0.000 \\
\hline A14-CR05-41 & CR05 & 41 & duplicate & $5 / 27 / 2003$ & $6 / 5 / 03$ & 0.000 & 0.000 & 0.000 & 0.000 & 0.000 \\
\hline A14-CR05-042 & CR05 & 42 & sample & $5 / 27 / 2003$ & $5 / 30 / 2003$ & 0.000 & 0.000 & 0.000 & 0.000 & 0.000 \\
\hline A14-CR05-042 & CR05 & 42 & duplicate & $5 / 27 / 2003$ & $7 / 14 / 03$ & 0.000 & 0.000 & 0.000 & 0.000 & 0.000 \\
\hline A14-CR05-043 & CR05 & 43 & sample & $5 / 27 / 2003$ & $5 / 30 / 2003$ & 0.000 & 0.000 & 0.000 & 0.000 & 0.000 \\
\hline A14-CR05-043 & CR05 & 43 & duplicate & $5 / 27 / 2003$ & $7 / 14 / 03$ & 0.000 & 0.000 & 0.000 & 0.000 & 0.000 \\
\hline A14-CR05-044 & CR05 & 44 & sample & $5 / 27 / 2003$ & $5 / 30 / 2003$ & 0.000 & 0.000 & 0.000 & 0.000 & 0.000 \\
\hline A14-CR05-044 & CR05 & 44 & duplicate & $5 / 27 / 2003$ & $7 / 14 / 03$ & 0.000 & 0.000 & 0.000 & 0.000 & 0.000 \\
\hline A14-CR05-045 & CR05 & 45 & sample & $5 / 27 / 2003$ & $5 / 30 / 2003$ & 0.000 & 0.000 & 0.000 & 0.000 & 0.000 \\
\hline A14-CR05-045 & CR05 & 45 & duplicate & $5 / 27 / 2003$ & $7 / 14 / 03$ & 0.000 & 0.000 & 0.000 & 0.000 & 0.000 \\
\hline A14-CR05-046 & CR05 & 46 & sample & $5 / 27 / 2003$ & $5 / 30 / 2003$ & 0.000 & 0.000 & 0.000 & 0.000 & 0.000 \\
\hline A14-CR05-046 & CR05 & 46 & duplicate & $5 / 27 / 2003$ & $7 / 14 / 03$ & 0.000 & 0.000 & 0.000 & 0.000 & 0.000 \\
\hline
\end{tabular}




\begin{tabular}{|c|c|c|c|c|c|c|c|c|c|c|}
\hline Sample ID & Boring & Depth (ft) & Type & $\begin{array}{c}\text { Date } \\
\text { Collected }\end{array}$ & $\begin{array}{c}\text { Date } \\
\text { Analyzed }\end{array}$ & $\begin{array}{c}\text { PCE } \\
(\mathrm{mg} / \mathrm{kg})\end{array}$ & $\begin{array}{c}\text { TCE } \\
(\mathrm{mg} / \mathrm{kg})\end{array}$ & $\begin{array}{l}\text { C-DCE } \\
(\mathrm{mg} / \mathrm{kg})\end{array}$ & $\begin{array}{c}\text { TCA } \\
(\mathrm{mg} / \mathrm{kg})\end{array}$ & $\begin{array}{l}\text { 11DCE } \\
(\mathrm{mg} / \mathrm{kg})\end{array}$ \\
\hline A14-CR05-047 & CR05 & 47 & sample & $5 / 27 / 2003$ & $5 / 30 / 2003$ & 0.000 & 0.000 & 0.000 & 0.000 & 0.000 \\
\hline A14-CR05-047 & CR05 & 47 & duplicate & $5 / 27 / 2003$ & $7 / 14 / 03$ & 0.000 & 0.000 & 0.000 & 0.000 & 0.000 \\
\hline A14-CR05-048 & CR05 & 48 & sample & $5 / 27 / 2003$ & $5 / 30 / 2003$ & 0.000 & 0.000 & 0.000 & 0.000 & 0.000 \\
\hline A14-CR05-048 & CR05 & 48 & duplicate & $5 / 27 / 2003$ & $7 / 14 / 03$ & 0.000 & 0.000 & 0.000 & 0.000 & 0.000 \\
\hline A14-CR05-049 & CR05 & 49 & sample & $5 / 27 / 2003$ & $5 / 30 / 2003$ & 0.000 & 0.000 & 0.000 & 0.000 & 0.000 \\
\hline 14-CR05-049 & CR05 & 49 & duplicate & $5 / 27 / 2003$ & $7 / 14 / 03$ & 0.000 & 0.000 & 0.000 & 0.000 & 0.000 \\
\hline A14-CRC & CR05 & 50 & sample & $5 / 27 / 2003$ & $5 / 30 / 2003$ & 0.000 & 0.000 & 0.000 & 0.000 & 0.000 \\
\hline A14-CR05-050 & CR05 & 50 & duplicate & $5 / 27 / 2003$ & $7 / 14 / 03$ & 0.000 & 0.000 & 0.000 & 0.000 & 0.000 \\
\hline A14-CR05-051 & CR05 & 51 & sample & $5 / 27 / 2003$ & $5 / 30 / 2003$ & 0.000 & 0.000 & 0.000 & 0.000 & 0.000 \\
\hline A14-CR05-051 & CR05 & 51 & duplicate & $5 / 27 / 2003$ & $7 / 14 / 03$ & 0.000 & 0.000 & 0.000 & 0.000 & 0.000 \\
\hline A14-CR05-052 & CR05 & 52 & sample & $5 / 27 / 2003$ & $5 / 30 / 2003$ & 0.000 & 0.000 & 0.000 & 0.000 & 0.000 \\
\hline A14-CR05-052 & CR05 & 52 & duplicate & $5 / 27 / 2003$ & $7 / 14 / 03$ & 0.000 & 0.000 & 0.000 & 0.000 & 0.000 \\
\hline A14-CR05-053 & CR05 & 53 & sample & $5 / 27 / 2003$ & $5 / 30 / 2003$ & 0.000 & 0.000 & 0.000 & 0.000 & 0.000 \\
\hline A14-CR05-053 & CR05 & 53 & duplicate & $5 / 27 / 2003$ & $7 / 14 / 03$ & 0.008 & 0.000 & 0.000 & 0.000 & 0.000 \\
\hline A14-CR05-054 & CR05 & 54 & sample & $5 / 27 / 2003$ & $5 / 30 / 2003$ & 0.023 & 0.000 & 0.000 & 0.000 & 0.000 \\
\hline A14-CRC & CR05 & 54 & duplicate & $5 / 27 / 2003$ & $7 / 14 / 03$ & 0.019 & 0.000 & 0.000 & 0.000 & 0.000 \\
\hline A14-CR05-055 & CR05 & 55 & sample & $5 / 27 / 2003$ & $5 / 30 / 2003$ & 0.295 & 0.000 & 0.000 & 0.000 & 0.000 \\
\hline A14-CR05-055 & CR05 & 55 & duplicate & $5 / 27 / 2003$ & $7 / 14 / 03$ & 0.132 & 0.000 & 0.000 & 0.000 & 0.000 \\
\hline A14-CR05-056 & CR05 & 56 & sample & $5 / 27 / 2003$ & $5 / 30 / 2003$ & 1.686 & 0.003 & 0.000 & 0.000 & 0.000 \\
\hline A14-CR05-056 & CR05 & 56 & duplicate & $5 / 27 / 2003$ & $7 / 14 / 03$ & 0.731 & 0.000 & 0.000 & 0.000 & 0.000 \\
\hline A14-CR05-057 & CR05 & 57 & sample & $5 / 27 / 2003$ & $5 / 30 / 2003$ & 37.334 & 0.013 & 0.000 & 0.000 & 0.000 \\
\hline A14-CR05-057 & CR05 & 57 & duplicate & $5 / 27 / 2003$ & $7 / 14 / 03$ & 28.073 & 0.007 & 0.000 & 0.000 & 0.000 \\
\hline A14-CR05-058 & CR05 & 58 & sample & $5 / 27 / 2003$ & $5 / 30 / 2003$ & 3925.067 & 0.986 & 2.132 & 0.000 & 0.000 \\
\hline A14-CR05-058 & CR05 & 58 & duplicate & $5 / 27 / 2003$ & $7 / 14 / 03$ & 1158.219 & 0.471 & 0.000 & 0.000 & 0.000 \\
\hline A14-CR05-059 & CR05 & 59 & sample & $5 / 28 / 2003$ & $5 / 30 / 2003$ & 2107.337 & 0.479 & 0.000 & 0.000 & 0.000 \\
\hline A14-CR05-059 & CR05 & 59 & duplicate & $5 / 28 / 2003$ & $7 / 14 / 03$ & 1215.932 & 0.314 & 0.000 & 0.000 & 0.000 \\
\hline A14-CR05-060 & CR05 & 60 & sample & $5 / 28 / 2003$ & $5 / 30 / 2003$ & 911.015 & 0.073 & 1.805 & 0.000 & 0.000 \\
\hline A14-CR05-060 & CR05 & 60 & duplicate & $5 / 28 / 2003$ & $7 / 14 / 03$ & 972.395 & 0.170 & 0.000 & 0.000 & 0.000 \\
\hline A14-CR05-061 & CR05 & 61 & \begin{tabular}{|l|} 
sample \\
\end{tabular} & $5 / 28 / 2003$ & $5 / 30 / 2003$ & 2.332 & 0.000 & 2.479 & 0.000 & 0.000 \\
\hline A14-CR05-061 & CR05 & 61 & duplicate & $5 / 28 / 2003$ & $7 / 14 / 03$ & 1.752 & 0.002 & 0.000 & 0.000 & 0.000 \\
\hline A14-CR05-062 & CR05 & 62 & sample & $5 / 28 / 2003$ & $5 / 30 / 2003$ & 0.395 & 0.144 & 0.000 & 0.000 & 0.000 \\
\hline
\end{tabular}




\begin{tabular}{|c|c|c|c|c|c|c|c|c|c|c|}
\hline Sample ID & Boring & Depth (ft) & Type & $\begin{array}{c}\text { Date } \\
\text { Collected }\end{array}$ & $\begin{array}{c}\text { Date } \\
\text { Analyzed }\end{array}$ & $\begin{array}{c}\text { PCE } \\
(\mathrm{mg} / \mathrm{kg})\end{array}$ & $\begin{array}{c}\text { TCE } \\
(\mathrm{mg} / \mathrm{kg})\end{array}$ & $\begin{array}{l}\text { C-DCE } \\
(\mathrm{mg} / \mathrm{kg})\end{array}$ & $\begin{array}{c}\text { TCA } \\
(\mathrm{mg} / \mathrm{kg})\end{array}$ & $\begin{array}{l}\text { 11DCE } \\
(\mathrm{mg} / \mathrm{kg})\end{array}$ \\
\hline A14-CR05-062 & CR05 & 62 & duplicate & $5 / 28 / 2003$ & $7 / 14 / 03$ & 0.203 & 0.000 & 0.000 & 0.000 & 0.000 \\
\hline A14-CR05-063 & CR05 & 63 & sample & $5 / 28 / 2003$ & $5 / 30 / 2003$ & 0.700 & 0.099 & 0.000 & 0.000 & 0.000 \\
\hline A14-CR05-063 & CR05 & 63 & duplicate & $5 / 28 / 2003$ & $7 / 14 / 03$ & 0.067 & 0.000 & 0.000 & 0.000 & 0.000 \\
\hline A14-CR05-065 & CR05 & 65 & sample & $5 / 28 / 2003$ & $5 / 30 / 2003$ & 0.575 & 0.098 & 0.000 & 0.000 & 0.000 \\
\hline A14-CR05-065 & CR05 & 65 & duplicate & $5 / 28 / 2003$ & $7 / 14 / 03$ & 0.094 & 0.000 & 0.000 & 0.000 & 0.000 \\
\hline A14-CR05-066 & CR05 & 66 & sample & $5 / 28 / 2003$ & $5 / 30 / 2003$ & 0.817 & 0.139 & 0.000 & 0.000 & 0.000 \\
\hline A14-CR05-066 & CR05 & 66 & duplicate & $5 / 28 / 2003$ & $7 / 14 / 03$ & 0.065 & 0.000 & 0.000 & 0.000 & 0.000 \\
\hline A14-CR05-067 & CR05 & 67 & sample & $5 / 28 / 2003$ & $5 / 30 / 2003$ & 1.216 & 0.509 & 0.000 & 0.000 & 0.000 \\
\hline A14-CR05-067 & CR05 & 67 & duplicate & $5 / 28 / 2003$ & $7 / 14 / 03$ & 0.040 & 0.000 & 0.000 & 0.000 & 0.000 \\
\hline A14-CR05-068 & CR05 & 68 & sample & $5 / 28 / 2003$ & $5 / 30 / 2003$ & 1.488 & 0.111 & 0.000 & 0.000 & 0.000 \\
\hline A14-CR05-068 & CR05 & 68 & duplicate & $5 / 28 / 2003$ & $7 / 14 / 03$ & 0.033 & 0.000 & 0.000 & 0.000 & 0.000 \\
\hline A14-CR05-069 & CR05 & 69 & sample & $5 / 28 / 2003$ & $5 / 30 / 2003$ & 0.547 & 0.000 & 0.000 & 0.000 & 0.000 \\
\hline A14-CR05-069 & CR05 & 69 & duplicate & $5 / 28 / 2003$ & $7 / 14 / 03$ & 0.373 & 0.000 & 0.000 & 0.000 & 0.000 \\
\hline A14-CR05-071 & CR05 & 71 & sample & $5 / 28 / 2003$ & $5 / 30 / 2003$ & 0.124 & 0.000 & 0.000 & 0.000 & 0.000 \\
\hline $\mathrm{A} 14-\mathrm{CRC}$ & CR05 & 71 & duplicate & $5 / 28 / 2003$ & $7 / 14 / 03$ & 0.122 & 0.000 & 0.000 & 0.000 & 0.000 \\
\hline A14-CR05-072 & CR05 & 72 & sample & $5 / 28 / 2003$ & $5 / 30 / 2003$ & 0.008 & 0.000 & 0.000 & 0.000 & 0.000 \\
\hline A14-CR05-072 & CR05 & 72 & duplicate & $5 / 28 / 2003$ & $7 / 14 / 03$ & 0.013 & 0.000 & 0.000 & 0.000 & 0.000 \\
\hline A14-CR05-073 & CR05 & 73 & sample & $5 / 28 / 2003$ & $5 / 30 / 2003$ & 0.007 & 0.000 & 0.000 & 0.000 & 0.000 \\
\hline $\mathrm{A} 14-\mathrm{CR}$ & CR05 & 73 & duplicate & $5 / 28 / 2003$ & $7 / 14 / 03$ & 0.010 & 0.000 & 0.000 & 0.000 & 0.000 \\
\hline A14-CRC & CR05 & 74 & sample & $5 / 28 / 2003$ & $5 / 30 / 2003$ & 0.006 & 0.000 & 0.000 & 0.000 & 0.000 \\
\hline A14-CR05-074 & CR05 & 74 & duplicate & $5 / 28 / 2003$ & $7 / 14 / 03$ & 0.007 & 0.000 & 0.000 & 0.000 & 0.000 \\
\hline A14-CR05-080 & CR05 & 80 & sample & $5 / 28 / 2003$ & $5 / 30 / 2003$ & 0.019 & 0.000 & 0.000 & 0.000 & 0.000 \\
\hline A14-CR05-080 & CR05 & 80 & duplicate & $5 / 28 / 2003$ & $7 / 14 / 03$ & 0.012 & 0.000 & 0.000 & 0.000 & 0.000 \\
\hline A14-CRC & CR05 & 81 & sample & $5 / 28 / 2003$ & $5 / 30 / 2003$ & 0.000 & 0.000 & 0.000 & 0.000 & 0.000 \\
\hline A14-CR05-081 & CR05 & 81 & duplicate & $5 / 28 / 2003$ & $7 / 14 / 03$ & 0.004 & 0.000 & 0.000 & 0.000 & 0.000 \\
\hline A14-CR05-090 & CR05 & 90 & sample & $5 / 28 / 2003$ & $5 / 30 / 2003$ & 0.007 & 0.000 & 0.000 & 0.000 & 0.000 \\
\hline A14-CR05-090 & CR05 & 90 & duplicate & $5 / 28 / 2003$ & $7 / 14 / 03$ & 0.020 & 0.000 & 0.000 & 0.000 & 0.000 \\
\hline A14-CRC & CR05 & 91 & sample & $5 / 28 / 2003$ & $5 / 30 / 2003$ & 0.007 & 0.000 & 0.000 & 0.000 & 0.000 \\
\hline A14-CR05-091 & CR05 & 91 & duplicate & $5 / 28 / 2003$ & $7 / 14 / 03$ & 0.009 & 0.000 & 0.000 & 0.000 & 0.000 \\
\hline A14-CR05-100 & CR05 & 100 & sample & $5 / 28 / 2003$ & $5 / 30 / 2003$ & 0.003 & 0.000 & 0.000 & 0.000 & 0.000 \\
\hline A14-CR05-100 & CR05 & 100 & duplicate & $5 / 28 / 2003$ & $7 / 14 / 03$ & 0.006 & 0.000 & 0.000 & 0.000 & 0.000 \\
\hline
\end{tabular}


WSRC-TR-2003-00540

September 2003

\begin{tabular}{|c|c|c|c|c|c|c|c|c|c|c|}
\hline Sample ID & Boring & Depth (ft) & Type & $\begin{array}{c}\text { Date } \\
\text { Collected }\end{array}$ & $\begin{array}{c}\text { Date } \\
\text { Analyzed }\end{array}$ & $\begin{array}{c}\text { PCE } \\
(\mathrm{mg} / \mathrm{kg})\end{array}$ & $\begin{array}{c}\text { TCE } \\
(\mathrm{mg} / \mathrm{kg})\end{array}$ & $\begin{array}{c}\text { C-DCE } \\
(\mathrm{mg} / \mathrm{kg})\end{array}$ & $\begin{array}{c}\text { TCA } \\
(\mathrm{mg} / \mathrm{kg})\end{array}$ & $\begin{array}{c}11 D C E \\
(\mathrm{mg} / \mathrm{kg})\end{array}$ \\
\hline A14-CR05-101 & CR05 & 101 & sample & $5 / 28 / 2003$ & $5 / 30 / 2003$ & 0.000 & 0.000 & 0.000 & 0.000 & 0.000 \\
\hline A14-CR05-101 & CR05 & 101 & duplicate & $5 / 28 / 2003$ & $7 / 14 / 03$ & 0.020 & 0.000 & 0.000 & 0.000 & 0.000 \\
\hline
\end{tabular}

